\title{
Permutation groups with small orbit growth
}

\author{
Manuel Bodirsky and Bertalan Bodor \\ Communicated by Christopher W. Parker
}

\begin{abstract}
Let $\mathcal{K}_{\exp }+$ be the class of all structures $\mathfrak{U}$ such that the automorphism group of $\mathfrak{U}$ has at most $c n^{d n}$ orbits in its componentwise action on the set of $n$-tuples with pairwise distinct entries, for some constants $c, d$ with $d<1$. We show that $\mathcal{K}_{\exp +}$ is precisely the class of finite covers of first-order reducts of unary structures, and also that $\mathcal{K}_{\exp +}$ is precisely the class of first-order reducts of finite covers of unary structures. It follows that the class of first-order reducts of finite covers of unary structures is closed under taking model companions and model-complete cores, which is an important property when studying the constraint satisfaction problem for structures from $\mathcal{K}_{\exp +}$. We also show that Thomas' conjecture holds for $\mathcal{K}_{\exp +}$ : all structures in $\mathcal{K}_{\exp +}$ have finitely many first-order reducts up to first-order interdefinability.
\end{abstract}

\section{Introduction}

A first-order reduct of a structure $\mathfrak{U}$ is a relational structure with the same domain as $\mathfrak{U}$ whose relations are first-order definable over $\mathfrak{U}$. Simon Thomas conjectured that every homogeneous structure $\mathfrak{U}$ with finite relational signature has only finitely many first-order reducts up to first-order interdefinability [64]. The conjecture has been verified for many famous homogeneous structures $\mathfrak{A}$ : e.g., for the ordered rationals [28], the countably infinite random graph [64], the homogeneous universal $K_{n}$-free graphs [65], the expansion of $(\mathbb{Q} ;<)$ by a constant [50], the universal homogeneous partial order [59], and the random ordered graph [20], and many more $[1,2,11,13]$. If we drop the assumption that the signature of the homogeneous structure $\mathfrak{A}$ is relational, then the conjecture of Thomas is false even if we keep the assumption that $\mathfrak{U}$ is $\omega$-categorical: already the countable atomless Boolean algebra has infinitely many first-order reducts [23].

Thomas' conjecture highlights our limited understanding of the class of homogeneous structures $\mathfrak{U}$ with finite relational signature. One approach to widen our understanding is to study homogeneous structures for some fixed signature; for example, classifications exist for the class of all homogeneous tournaments [52],

The authors have received funding from the European Research Council (Grant Agreement no. 681988, CSP-Infinity). 
homogeneous undirected graphs [44], homogeneous partial orders [63], general homogeneous digraphs [35], homogeneous permutations [32], and homogeneous coloured multipartite graphs $[49,53]$. However, already the class of homogeneous 3-uniform hypergraphs appears to be very difficult [5]. If we impose additional assumptions, e.g., that the age of $\mathfrak{U}$ can be described by finitely many forbidding substructures, we might hope for systematic understanding and effectiveness results for various questions. However, it is not clear how to use this assumption for proving that $\mathfrak{U}$ has finitely many first-order reducts.

Another approach to understanding the class of homogeneous structures, followed in this paper, is to start with the most symmetric structures in this class. Symmetry can be measured by the number of orbits $o_{n}^{i}(G)$ of the diagonal action of the automorphism group $G=\operatorname{Aut}(\mathfrak{U})$ on tuples from $A^{n}$ that have pairwise distinct entries. By the theorem of Engeler, Ryll-Nardzeski, and Svenonius, these orbits are in one-to-one correspondence with the model-theoretic types of $n$ pairwise distinct elements in $\mathfrak{A}$. Alternatively, we might count the number of orbits $o_{n}^{S}(G)$ of the action of $G$ on $n$-element subsets of $A$. The investigation of both of these measures has been pioneered by Cameron; see [30] for an introduction to the subject. The sequence $o_{n}^{i}(G)$ is linked to labeled enumeration problems, which are the most intensively studied counting problems in enumerative combinatorics, while $o_{n}^{S}(G)$ is linked to unlabeled enumeration problems. Many structural results about $G$ are available when we impose restrictions on $o_{n}^{s}(G)$; see, e.g., [54-56]. The present article, in contrast, focuses on restricting $o_{n}^{i}(G)$.

A structure $\mathfrak{A}$ is finite if and only if $o_{n}^{i}(\operatorname{Aut}(\mathfrak{A}))$ is eventually 0 . It is a by-product of our results that the class $\mathcal{K}_{\exp }$ of all structures $\mathfrak{U}$ where $o_{n}^{i}(\operatorname{Aut}(\mathfrak{U}))$ grows at most exponentially equals the class of first-order reducts of unary structures; by a unary structure, we mean any at most countable structure with finitely many unary relations. Our main result pushes this further: we study the class $\mathcal{K}_{\text {exp+ }}$ of structures $\mathfrak{U}$ such that $o_{n}^{i}(\operatorname{Aut}(\mathfrak{Q}))$ is bounded by $c n^{d n}$ for some constants $c, d$ with $d<1$. Note that, for example, the structure $(\mathbb{Q} ;<)$ does not belong to $\mathcal{K}_{\exp +}$ because $o_{n}^{i}(\mathbb{Q} ;<)=n$ !. Also, $\mathcal{K}_{\exp +}$ contains no structure $\mathfrak{A}$ with a definable equivalence relation with infinitely many infinite classes because $o_{n}^{i}(\mathfrak{U})$ would in this case be at least as large as the $n$-th Bell number, which grows asymptotically faster than $c n^{d n}$ (see Lemma 6.1). We show that $\mathcal{K}_{\exp +}$ contains precisely those structures that are finite covers of first-order reducts of unary structures (see Theorem 6.29).

Finite covers in model theory and infinite permutation groups have been studied in the context of classifying totally categorical structures $[4,47,48]$ and, more generally, for studying $\omega$-categorical $\omega$-stable structures [33,34]. Finite covers became an important topic in its own $[38,40,61]$; we refer to the survey article for an introduction [39]. It follows from our result that the class of finite covers of 
reducts of unary structures equals the class of first-order reducts of finite covers of unary structures. Using the terminology of [39], we show that all finite covers of unary structures split, but not necessarily strongly. All structures in $\mathcal{K}_{\exp +}$ can be expanded to structures that are homogeneous in a finite relational language, and we show that they all satisfy Thomas' conjecture (see Theorem 6.37). The proof uses a result of Macpherson which implies that structures in $\mathcal{K}_{\exp }+$ which have a primitive automorphism group must be highly transitive [55].

The class $\mathcal{K}_{\text {exp}}+$ can be seen as the "smallest reasonably robust class that contains all finite structures as well as some infinite ones" (for formalisations of this statement, see Section 8.3). So, whenever a statement that holds for all finite structures needs to be generalised to a class of "slightly infinite structures", it might be a good idea to try to first prove the statement for $\mathcal{K}_{\exp +}$. This is precisely the situation for the constraint satisfaction problem.

\subsection{Complexity of constraint satisfaction}

Let $\mathfrak{B}$ be a structure with finite relational signature. The constraint satisfaction problem for $\mathfrak{B}$ is the computational problem of deciding whether a given finite structure $\mathfrak{A}$ with the same signature as $\mathfrak{B}$ has a homomorphism to $\mathfrak{B}$. For finite structures $\mathfrak{B}$, Feder and Vardi [41] conjectured that the computational complexity of CSP( $(\mathfrak{B})$ satisfies a dichotomy: it is either in P or NP-complete. Using concepts and techniques from universal algebra, Bulatov and Zhuk recently presented independent proofs of this conjecture $[27,66]$.

The universal-algebraic approach can also be applied when $\mathfrak{B}$ is countably infinite and $\omega$-categorical. In this case, the computational complexity of $\mathfrak{B}$ is captured by the polymorphism clone of $\mathfrak{B}$ (see [16]), which can be seen as a generalisation of the automorphism group of $\mathfrak{B}$ : it consists of all homomorphisms from $\mathfrak{B}^{n}$ to $\mathfrak{B}$, for $n \in \mathbb{N}$. Moreover, every $\omega$-categorical structure $\mathfrak{B}$ is homomorphically equivalent to an (up to isomorphism unique) structure $\mathfrak{C}$ with the property that the automorphisms of $\mathfrak{C}$ lie dense in the endomorphisms of $\mathfrak{C}$, called the model-complete core of $\mathfrak{B}$. The model-complete core $\mathfrak{C}$ of $\mathfrak{B}$ is again $\omega$-categorical, and has the same CSP as $\mathfrak{B}$, so that we prefer to analyse $\mathfrak{C}$ rather than $\mathfrak{B}$. This simplification of the classification problem is a key step for many results (see, e.g., $[6,14,18]$ ), including the finite-domain classification $[27,66]$.

Therefore, if we want to classify the computational complexity of CSP( $\mathfrak{B})$ for all structures $\mathfrak{B}$ from a class $\mathcal{C}$, it is important whether the class $\mathcal{C}$ is closed under the formation of model-complete cores. When $\mathfrak{C}$ is the model-complete core of $\mathfrak{B}$, then it is easy to see that $o_{n}^{i}(\mathfrak{C}) \leq o_{n}^{i}(\mathfrak{B})$; hence, in particular, the classes $\mathcal{K}_{\exp }$ and $\mathcal{K}_{\exp }+$ are closed under taking model-complete cores. This makes these classes attractive goals for extending the mentioned dichotomy result from finite domains. 
As mentioned before, our results imply that every structure in $\mathcal{K}_{\text {exp }}$ is a firstorder reduct of a unary structure. For those structures, it has already been shown that they are in P or NP-complete [15] (using the mentioned dichotomy for finitedomain CSPs). Our main result states that $\mathcal{K}_{\exp +}$ is precisely the class of first-order reducts of finite covers of unary structures. For classifying the complexity of the CSP for all structures in this class, our result implies that we can assume without loss of generality that these structures are model-complete cores. We thus see our result as a first step towards classifying the CSP for first-order reducts of finite covers of unary structures.

\subsection{Definable sets with atoms}

In theoretical computer science, one is interested in finite representations of infinite structures; one approach to this is the framework of definable sets and computation with atoms $[24,25]$. This leads to new models of computation over infinite structures with interesting links to long-standing open problems in finite model theory, namely the question whether there is a logic for $\mathrm{P}$ and computation in choiceless polynomial time [26].

If the "atom structure" is $(\mathbb{N} ;=)$ (which is besides $(\mathbb{Q} ;<$ ) the most frequently used base structure in this area), then definable sets (in this case also studied under the name nominal sets [42]) correspond precisely to the class $\mathcal{K}=$ of structures that are first-order interpretable over $(\mathbb{N} ;=)$ in the sense of model theory (for an explicit discussion of the connection, see [51, Lemma 7 and the remarks thereafter]). The class $\mathcal{K}=$ might appear to be trivial to many model theorists (all structures in it are $\omega$-categorical, $\omega$-stable, and they are first-order reducts of homogeneous finitely bounded structures), but in fact, many questions about this class remain open; see Section 10 for a small sample of open problems. It follows from our results (see Remark 6.30) that $\mathcal{K}_{\exp +} \subseteq \mathcal{K}_{=}$, and we can answer for $\mathcal{K}_{\exp +\text { many }}$ questions that we cannot answer for the class $\mathcal{K}=$ in general. So our results can also be seen as a first step towards a better understanding of $\mathcal{K}_{=}$.

\section{Preliminaries}

If $\sim$ is an equivalence relation on $X$ and $x \in X$, then $[x]_{\sim}$ denotes the equivalence class of $x$ with respect to $\sim$, and $X / \sim:=\{[x] \sim \mid x \in X\}$ denotes the set of all $\sim$-classes. We write $|\sim|$ for $|X / \sim|$. If $\sim_{1}$ and $\sim_{2}$ are equivalence relations on $X$, then we say that $\sim_{1}$ is finer than $\sim_{2}$ (or $\sim_{2}$ is coarser than $\sim_{1}$ ) if $\sim_{1}$ is contained in $\sim_{2}$ (as binary relations). 


\subsection{Permutation group notation}

When $G$ is a group, we write $H \leq G$ if $H$ is a subgroup of $G$, and $H \triangleleft G$ if $H$ is a normal subgroup of $G$. We write $[G: H]$ for the index of $H$ in $G$. For any set $X$, we write $\operatorname{Sym}(X)$ for the group of all permutations of $X$. If $G \leq \operatorname{Sym}(X)$ and $x \in X$, then $G_{x}$ denotes the stabiliser of the element $x$. Let $Y \subseteq X$. Then

- $G_{Y}$ denotes the pointwise stabiliser, and

- $G_{\{Y\}}$ denotes the setwise stabiliser of the set $Y$.

- $\left.G\right|_{Y}$ denotes the restriction of $G$ to $Y$ provided that $Y$ is preserved by $G$.

If $Y$ is finite, say $Y=\left\{x_{1}, \ldots, x_{n}\right\}$, then we also use the notation $G_{x_{1}, \ldots, x_{n}}$ for the pointwise stabiliser of the set $Y$. Let $G$ be a permutation group on $X$. An orbit of $G$ is a set of the form $\{g(x) \mid g \in G\}$ for some $x \in X$. The algebraic closure of $Y \subseteq X$ with respect to $G$ is the union of the finite orbits of $G_{Y}$, and it is denoted by $\operatorname{acl}_{G}(Y)$. If $x \in X$, then we use the notation $\operatorname{acl}_{G}(x)$ instead of $\operatorname{acl}_{G}(\{x\})$. It is well known that $\operatorname{acl}_{G}$ is a closure operator on the subsets of $X$, and in particular, we have $\operatorname{acl}_{G}\left(\operatorname{acl}_{G}(Y)\right)=\operatorname{acl}_{G}(Y)$ for all $Y \subseteq X$. If the group $G$ is clear from the context, then we will omit the subscript from this notation.

An equivalence relation $\sim$ of $X$ is called a congruence of a permutation group $G \subseteq \operatorname{Sym}(X)$ if $x \sim y$ and $g \in G$ implies $g(x) \sim g(y)$ for all $x, y \in X$ and $g \in G$. In other words, an equivalence relation $\sim$ is a congruence if the corresponding partition is $G$-invariant. Every permutation group $G \subseteq \operatorname{Sym}(X)$ has two trivial congruences, namely $X^{2}$ and $\{(x, x) \mid x \in X\}$. We call the former the universal congruence and the latter the identity congruence. If $\sim$ is a congruence of some permutation group $G \subseteq \operatorname{Sym}(X)$, then $G$ acts naturally on $X / \sim$. The image of this action, as a subgroup of $\operatorname{Sym}(X / \sim)$, is denoted by $G / \sim$.

Definition 2.1. Let $\pi: A \rightarrow B$ be a map. We write $\sim_{\pi}$ for the equivalence relation $\left\{\left(a_{1}, a_{2}\right) \mid \pi\left(a_{1}\right)=\pi\left(a_{2}\right)\right\}$ on $A$. If $G$ is a permutation group on $A$ such that $\sim_{\pi}$ is a congruence of $G$, then $\pi$ gives rise to a homomorphism $\mu_{\pi}: G \rightarrow \operatorname{Sym}(B)$ defined by $\mu_{\pi}(g)(a):=\pi\left(g\left(\pi^{-1}(a)\right)\right)$ (this is well-defined since $G$ preserves $\sim_{\pi}$ ).

\subsection{Direct products}

Let $I$ be a set. For each $i \in I$, let $A_{i}$ be a group. Then $\prod_{i \in I} A_{i}$ denotes the direct product of the $A_{i}$; i.e., the elements have the form $\left(a_{i}\right)_{i \in I}$ for $a_{i} \in A_{i}$, and group composition is defined pointwise. When the $A_{i}$ are permutation groups on disjoint sets $X_{i}$ for every $i \in I$, then $A:=\prod_{i \in I} A_{i}$ acts naturally (intransitively) on $X:=\bigsqcup_{i \in I} X_{i}$ as follows: for $\alpha \in A$ and $x \in X$, define $\alpha(x):=\alpha_{i}(x)$ if $x \in X_{i}$. 
It is easy to see that if each of the $A_{i}$ is closed in $\operatorname{Sym}\left(X_{i}\right)$, then the permutation group defined by the action of $A$ on $X$ is closed in $\operatorname{Sym}(X)$, and hence is the automorphism group of some relational structure with domain $X$.

\subsection{Wreath products}

Let $A$ be a group acting on the set $F$, and let $Y$ be a set. Let $H$ be a group acting on $Y$, and let $X:=F \times Y$. Then there are natural actions of the groups $N:=\prod_{y \in Y} A$ and $H$ on the set $X$, defined as follows.

(1) If $\alpha \in N$ and $(f, y) \in X$, then $\alpha(f, y):=\left(\alpha_{y}(f), y\right)$,

(2) If $\beta \in H$ and $(f, y) \in X$, then $\beta(f, y):=(f, \beta(y))$.

Let $G$ be the subgroup of $\operatorname{Sym}(X)$ generated by the actions of $N$ and of $H$ on $X$; we view $N$ and $H$ as subsets of $G$. If $\alpha \in N$ and $\beta \in H$, then

$$
\beta^{-1} \alpha \beta(f, y)=\beta^{-1} \alpha(f, \beta(y))=\beta^{-1}\left(\alpha_{\beta(y)}(f), \beta(y)\right)=\left(\alpha_{\beta(y)}(f), y\right),
$$

so $\beta^{-1} \alpha \beta \in N$ and $N \triangleleft G$. Then $G=N H$ and $N \cap H=\left\{\operatorname{id}_{X}\right\}$. Hence, the group $G$ can be written as the semidirect product $\prod_{y \in Y} A \rtimes H$. The group $G$ is called the wreath product of the groups $A$ and $H$ (with its canonical imprimitive action on $X$ ) and will be denoted by $A$ ? $H$.

\subsection{Interdefinability, bi-definability, bi-interpretability}

We write $A, B, C$ for the domains of the structures $\mathfrak{A}, \mathfrak{B}, \mathfrak{C}$, respectively. If $G$ is a set of permutations on a set $A$, then $\operatorname{Inv}(G)$ denotes the relational structure $\mathfrak{A}$ with domain $A$ which carries all relations that are preserved by all permutations of $G$. The operations Aut and Inv form a Galois connection between the set of all relational structures $\mathfrak{A}$ with domain $A$ and the set of sets of permutations $G$ on $A$ (see, e.g., [9]). The permutation group $\operatorname{Aut}(\operatorname{Inv}(G))$ is the smallest permutation group containing $G$ that is closed in $\operatorname{Sym}(A)$ equipped with the topology of pointwise convergence. This topology is the restriction of the product topology on $A^{A}$, where $A$ is taken to be discrete. A permutation group $G$ on $A$ is closed in $\operatorname{Sym}(A)$ if and only if $G$ is the automorphism group of a relational structure. If $\mathfrak{A}$ is $\omega$-categorical, then the structure $\operatorname{Inv}(\operatorname{Aut}(\mathfrak{H}))$ is the expansion of $\mathfrak{U}$ by all relations that can be defined by a first-order formula in $\mathfrak{U}$ (this is a consequence of the proof of the theorem of Ryll-Nardzewski; see [45]).

It follows that $\operatorname{Aut}(\mathfrak{U}) \subseteq \operatorname{Aut}\left(\mathfrak{U}^{\prime}\right)$ if and only if all relations of $\mathfrak{U}^{\prime}$ are first-order definable (without parameters) over $\mathfrak{U}$; in this case, we say that $\mathfrak{Q}^{\prime}$ is a first-order reduct of $\mathfrak{U}$. Two structures on the same domain are called interdefinable if they are reducts of one another. By the above, if $\mathfrak{A}$ or $\mathfrak{U}^{\prime}$ is $\omega$-categorical, then $\mathfrak{U}$ and $\mathfrak{U}^{\prime}$ are interdefinable if and only if $\operatorname{Aut}(\mathfrak{U})=\operatorname{Aut}\left(\mathfrak{U}^{\prime}\right)$. 
Two structures $\mathfrak{A}$ and $\mathfrak{B}$, not necessarily with the same domain, are called $b i$ definable if there exists a bijection $f: A \rightarrow B$ between the domains of $\mathfrak{A}$ and $\mathfrak{B}$ such that $\mathfrak{A}$ and $\mathfrak{B}$ are interdefinable after identifying $A$ and $B$ along $f$. It follows that two $\omega$-categorical structures $\mathfrak{U}$ and $\mathfrak{B}$ are bi-definable if and only if $\operatorname{Aut}(\mathfrak{U})$ and $\operatorname{Aut}\left(\mathfrak{U}^{\prime}\right)$ are isomorphic as permutation groups. For example, the structures $(\mathbb{Z} ;\{0\})$ and $(\mathbb{Z} ;\{1\})$ are bi-definable, but not interdefinable.

A (d-dimensional) interpretation of $\mathfrak{B}$ in $\mathfrak{A}$ is a partial surjective map $I$ from $A^{d}$ to $B$ such that the pre-image of $B$, of the equality relation on $B$, and of each relation of $\mathfrak{B}$ under $I$ is first-order definable in $\mathfrak{A}$. If $\mathfrak{A}$ has a $d$-dimensional firstorder interpretation $I$ in $\mathfrak{B}$ and $\mathfrak{B}$ has an $e$-dimensional first-order interpretation $J$ in $\mathfrak{A}$ such that the relation

$$
\left\{\left(x, y_{1,1}, \ldots, y_{d, e}\right) \mid x=J\left(I\left(y_{1,1}, \ldots, y_{d, 1}\right), \ldots, I\left(y_{1, e}, \ldots, y_{d, e}\right)\right)\right\}
$$

is first-order definable in $\mathfrak{B}$ and

$$
\left\{\left(x, y_{1,1}, \ldots, y_{d, e}\right) \mid x=I\left(J\left(y_{1,1}, \ldots, y_{1, e}\right), \ldots, J\left(y_{d, 1}, \ldots, y_{d, e}\right)\right)\right\}
$$

is first-order definable in $\mathfrak{A}$, then $\mathfrak{A}$ and $\mathfrak{B}$ are called bi-interpretable. By a result of Coquand, Ahlbrandt, and Ziegler [3], two $\omega$-categorical structures $\mathfrak{A}$ and $\mathfrak{B}$ are bi-interpretable if and only if $\operatorname{Aut}(\mathfrak{U})$ and $\operatorname{Aut}(\mathfrak{B})$ are topologically isomorphic, i.e., isomorphic via a mapping which is a homeomorphism with respect to the pointwise convergence topology.

\subsection{Orbit growth and some classes of structures}

Let $X$ be a countably infinite set. There are three natural counting sequences attached to a permutation group on $X$, introduced and discussed in general in [30, $31]$.

Definition 2.2. Let $G \subseteq \operatorname{Sym}(X)$ be a permutation group, and let $n \in \mathbb{N}$. Then

- $o_{n}(G)$ denotes the number of $n$-orbits of $G$, i.e., the number of orbits of the natural action $G \curvearrowright X^{n}$;

- $o_{n}^{i}(G)$ denotes the number of injective $n$-orbits of $G$, i.e., the number of orbits of the natural action $G \curvearrowright X^{(n)}$ where

$$
X^{(n)}:=\left\{\left(x_{1}, \ldots, x_{n}\right) \in X^{n} \mid x_{i} \neq x_{j} \text { for all distinct } i, j \in\{1, \ldots, n\}\right\} ;
$$

- $o_{n}^{s}(G)$ denotes the number of orbits of $n$-subsets of $G$, i.e., the number of orbits of the natural action $G \curvearrowright\left(\begin{array}{c}X \\ n\end{array}\right)\left(=\left\{Y \subset X^{n}|| Y \mid=n\right\}\right)$.

If $\mathfrak{U}$ is a structure, then let

$$
o_{n}(\mathfrak{U}):=o_{n}(\operatorname{Aut}(\mathfrak{U})), \quad o_{n}^{i}(\mathfrak{H}):=o_{n}^{i}(\operatorname{Aut}(\mathfrak{H})), \quad o_{n}^{s}(\mathfrak{H}):=o_{n}^{s}(\operatorname{Aut}(\mathfrak{H})) .
$$


In the notation above, we omit the reference to the group $G$ or the structure $\mathfrak{A}$ if it is clear from the context.

A permutation group is called transitive if $o_{i}(G)=1$ and highly transitive if $o_{i}(G)=1$ for all $i \in \mathbb{N}$.

Definition 2.3. A permutation group $G \subseteq \operatorname{Sym}(X)$ is called oligomorphic if $o_{n}(G)$ is finite for all $n$.

Clearly, in Definition 2.3, we could have equivalently required that $o_{n}^{i}$ or $o_{n}^{s}$ are finite for all $n$. By the theorem of Engeler, Ryll-Nardzewski, and Svenonius, a countably infinite relational structure $\mathfrak{A}$ is $\omega$-categorical if and only if $\operatorname{Aut}(\mathfrak{A})$ is oligomorphic (see for instance [45]). In this paper, we are particularly interested in the following classes of structures and permutation groups.

Definition 2.4. We introduce the following classes of permutation groups and structures.

- Let $\mathscr{E}_{\exp }$ denote the class of those permutation groups $G$ acting on a countable set $X$ for which there is a constant $c$ such that $o_{n}^{i}(G) \leq c^{n}$.

- Let $\mathcal{K}_{\text {exp }}$ denote the class of all countable structures $\mathfrak{A}$ with an automorphism group in $\mathcal{E}_{\text {exp }}$.

- Let $\mathcal{E}_{\text {exp }+}$ denote the class of those permutation groups $G$ acting on a countable set $X$ for which there are constants $c$ and $d<1$ such that $o_{n}^{i}(G) \leq c n^{d n}$.

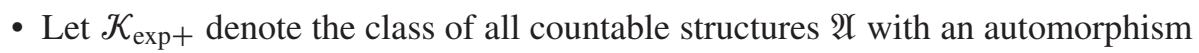
group in $\mathcal{G}_{\exp +}$.

Remark 2.5. Note that the conditions $G \in \mathscr{E}_{\exp }$ and $G \in \mathscr{E}_{\exp }+$ imply that $G$ is oligomorphic, and therefore $\mathfrak{A} \in \mathcal{K}_{\exp }$ and $\mathfrak{Q} \in \mathcal{K}_{\exp }+$ imply that $\mathfrak{A}$ is $\omega$-categorical.

We write $\mathbb{N}$ not only for the set of natural numbers, but also for the structure with the empty signature whose domain is $\mathbb{N}$.

Definition 2.6. We write

- 8 for the class of all at most countable structures that are first-order interdefinable with a structure having the empty signature,

- $U$ for the class of at most countable structures that are first-order interdefinable with a structure having a finite signature of unary relation symbols, 
- $U^{*}$ for the class of the structures $\mathfrak{U} \in \mathcal{U}$ such that every orbit of Aut( $(\mathfrak{H})$ is either a singleton or infinite.

When $\mathcal{C}$ is a class of structures, we write $\mathcal{C}_{\mathrm{nf}}$ for the class consisting of all the structures in $\mathcal{C}$ that have no finite orbits. Note that $\mathbb{N} \in \delta \subset U_{\mathrm{nf}} \subset U^{*} \subset U$ and that $\left(u^{*}\right)_{\mathrm{nf}}=u_{\mathrm{nf}}$.

\subsection{Congruences of oligomorphic groups}

We need the following easy observation about oligomorphic groups.

Proposition 2.7. Every oligomorphic permutation group has finitely many congruences.

Proof. Every congruence of a permutation group is a union of its 2-orbits. Then the claim follows directly from oligomorphicity.

Lemma 2.8. Let $G$ be an oligomorphic permutation group, and let $\sim$ be a congruence of $G$ which has finite equivalence classes. Then $a \sim b$ implies $b \in \operatorname{acl}_{G}(a)$.

Proof. Suppose that $a \sim b$, but $b \notin \operatorname{acl}(a)$. Then the orbit of $b$ in $G_{a}$ is infinite. Let $b^{\prime}$ be any element in this orbit. Then, by definition, $a \sim b^{\prime}$. Hence, the equivalence class of $a$ is infinite, a contradiction.

If $\sim_{1}$ and $\sim_{2}$ are congruences, then the inclusion-wise smallest congruence relation that contains both $\sim_{1}$ and $\sim_{2}$ is called the equivalence relation generated by $\sim_{1}$ and $\sim_{2}$.

Lemma 2.9. Let $G$ be an oligomorphic permutation group, and let $\sim_{1}$ and $\sim_{2}$ be congruences of $G$ with finite classes. Then the congruence generated by $\sim_{1}$ and $\sim_{2}$ also has finite classes.

Proof. Let $\sim$ be the congruence generated by $\sim_{1}$ and $\sim_{2}$, and suppose that $a \sim b$. Then there exists a sequence $a_{0}, b_{0}, a_{1}, b_{1}, \ldots, a_{k}, b_{k}$ with $a_{0}=a$ and $b_{k}=b$ such that $a_{i} \sim_{1} b_{i}$ for all $i \leq k$ and $b_{i} \sim_{2} a_{i+1}$ for all $i<k$. By Lemma 2.8, this implies that $b_{i} \in \operatorname{acl}\left(a_{i}\right)$ and $a_{i+1} \in \operatorname{acl}\left(b_{i}\right)$ for all $i$. Since acl is a closure operator, it follows that $b \in \operatorname{acl}(a)$. Since $G$ is oligomorphic, it follows that $\operatorname{acl}(a)$ is finite. Therefore, the equivalence class of $a$ is also finite.

Definition 2.10. Let $G$ be an oligomorphic permutation group. Then

- $\nabla(G)$ denotes the intersection of all congruences of $G$ with finitely many classes,

- $\Delta(G)$ denotes the smallest congruence that contains all congruences of $G$ with finite classes. 
If $\mathfrak{U}$ is an $\omega$-categorical structure, then we use the notation $\nabla(\mathfrak{U}):=\nabla(\operatorname{Aut}(\mathfrak{U}))$, and $\Delta(\mathfrak{U}):=\Delta(\operatorname{Aut}(\mathfrak{U}))$.

Remark 2.11. Since $G$ has finitely many congruences, it follows that $\nabla(G)$ also has finitely many classes, i.e., it is the finest congruence of $G$ with finitely many classes. By Lemma 2.9, it follows that every class of $\Delta(G)$ is finite, i.e., $\Delta(G)$ is the coarsest congruence of $G$ with finite classes.

Remark 2.12. If $x$ and $y$ are in the same orbit, then their $\Delta$-classes have the same size. If $G$ has finitely many orbits, it follows that there exists some $n \in \mathbb{N}$ such that all elements lie in a $\Delta$-class of size at most $n$.

The congruence $\Delta$ has the following equivalent description.

Lemma 2.13. Let $G$ be an oligomorphic permutation group on a countably infinite set $X$. Then $(x, y) \in \Delta(G)$ if and only if $y \in \operatorname{acl}_{G}(x)$ and $x \in \operatorname{acl}_{G}(y)$.

Proof. Let $\Delta^{\prime}(G)=\{(x, y) \mid y \in \operatorname{acl}(x) \wedge x \in \operatorname{acl}(y)\}$. We claim that $\Delta^{\prime}(G)$ is an equivalence relation. It is clear that $\Delta^{\prime}(G)$ is reflexive and symmetric. The transitivity follows from the fact that acl is a closure operator. It is also clear from the definition that $\Delta^{\prime}(G)$ is preserved by $G$. Hence, $\Delta^{\prime}(G)$ is a congruence. For any $x \in X$, we have $[x]_{\Delta^{\prime}(G)} \subseteq \operatorname{acl}_{G}(x)$, so every class of $\Delta^{\prime}(G)$ is finite. Therefore, $\Delta^{\prime}(G)$ is finer than $\Delta(G)$. On the other hand, if $(x, y) \in \Delta(G)$, then $y \in \operatorname{acl}(x)$ and $x \in \operatorname{acl}(y)$, and thus $(x, y) \in \Delta(G)$.

We often use the following observation throughout this text.

Lemma 2.14. Let $G$ be an oligomorphic permutation group. Then every class of $\nabla(G)$ is either infinite or a singleton.

Proof. If the class of $x \in X$ is finite, then its orbit is also finite. Indeed, let $O$ be the orbit of $x$. Then every class of $\nabla(G)$ in $O$ is of the same size. So if this size is finite, then $O$ is also finite since $\nabla$ has finitely many classes.

Let $X_{\text {fin }}$ be the union of the finite orbits of $G$. By oligomorphicity, it follows that $X_{\text {fin }}$ is finite. Then $\nabla^{\prime}:=\nabla(G) \cap\left\{(x, x) \mid x \in X_{\text {fin }}\right\}$ is also a congruence of $\nabla(G)$. Since $X_{\text {fin }}$ is finite, the congruence $\nabla^{\prime}$ has finitely many classes. This implies that $\nabla^{\prime}=\nabla(G)$, and thus every class of $\nabla(G)$ within $X_{\text {fin }}$ is a singleton.

Lemma 2.15. Let $G$ be an oligomorphic permutation group on $X$, and let $\sim$ be a congruence of $G$ with finite classes. Then the congruence generated by $\sim$ and $\nabla(G)$ equals $\left\{(x, y) \in X^{2} \mid\left([x]_{\sim},[y]_{\sim}\right) \in \nabla(G / \sim)\right\}$. 
Proof. If $\pi: X \rightarrow X / \sim$ is the factor map $x \mapsto[x]_{\sim}$, and $\approx$ is a congruence of $G / \sim$, then

$$
\pi^{-1}(\approx):=\left\{(x, y) \in X^{2} \mid(\pi(x), \pi(y)) \in \approx\right\}
$$

is a congruence of $G$ which is coarser than $\sim$. In fact, $\pi^{-1}$ defines a bijection between the congruences of $G / \sim$ and those congruences of $G$ which are coarser than $\sim$. The congruence $\pi^{-1}(\nabla(G / \sim))$ has finitely many classes since $\nabla(G / \sim)$ has finitely many classes. Hence, $\pi^{-1}(\nabla(G / \sim)$ is the finest congruence of $G$ that is coarser than $\sim$ and has finitely many classes. So, by definition, it equals the congruence generated by $\sim$ and $\nabla(G)$.

\subsection{Finite covers}

We now introduce the concept of finite covers that plays a central role in this article. Forming finite covers may be viewed as a way to construct new $\omega$-categorical structures from known ones; a more appropriate way is to view them as a way to decompose $\omega$-categorical structures into (hopefully) simpler parts.

Definition 2.16. Let $\mathfrak{A}$ and $\mathfrak{B}$ be structures. A mapping $\pi: \mathfrak{A} \rightarrow \mathfrak{B}$ is called a finite covering map (or finite cover) if

(1) $\pi$ is surjective,

(2) for each $w \in B$, the set $\pi^{-1}(w)$ is finite,

(3) $\sim_{\pi}$ is preserved by $\operatorname{Aut}(\mathfrak{U})$,

(4) the image of $\operatorname{Aut}(\mathfrak{H})$ under $\mu_{\pi}$ equals $\operatorname{Aut}(\mathfrak{B})$.

(See Definition 2.1 for the definition of $\sim_{\pi}$ and $\mu_{\pi}$.) The sets $\pi^{-1}(w)$, for $w \in B$, are called the fibers of the finite covering map $\pi$. A structure $\mathfrak{A}$ is called a finite covering structure of $\mathfrak{B}$ if there is a finite covering map $\pi: \mathfrak{A} \rightarrow \mathfrak{B}$.

Remark 2.17. A finite covering structure of an $\omega$-categorical structure has an oligomorphic automorphism group, and hence is $\omega$-categorical.

Remark 2.18. Let $\mathfrak{U}$ be an arbitrary structure and $\sim$ a congruence of Aut( $\mathfrak{U})$. If all $\sim$-classes are finite, then $\mathfrak{A}$ is a finite covering structure of the quotient structure $\mathfrak{X} / \sim$, where $\mathfrak{X} / \sim$ can be any structure such that $\operatorname{Aut}(\mathfrak{H} / \sim)=\operatorname{Aut}(\mathfrak{H}) / \sim$. In fact, every finite covering structure is of this form. Indeed, let $\mathfrak{Q}$ be a structure. If $\pi: \mathfrak{U} \rightarrow \mathfrak{B}$ is a finite covering map, then $\sim_{\pi}$ is a congruence of Aut $(\mathfrak{H})$ and there is a natural bijection between $B$ and $A / \sim_{\pi}$ defined by $w \mapsto \pi^{-1}(w)$. Let us identify $B$ and of $A / \sim_{\pi}$ along this bijection, and let $\mathfrak{A} / \sim_{\pi}$ be any structure such that $\operatorname{Aut}\left(\mathfrak{H} / \sim_{\pi}\right)=\operatorname{Aut}(\mathfrak{B})$. The image of Aut( $(\mathfrak{U})$ under the homomorphism $\mu_{\pi}$ equals $\operatorname{Aut}(\mathfrak{B})$; hence $\operatorname{Aut}(\mathfrak{U}) / \sim_{\pi}=\operatorname{Aut}(\mathfrak{B})=\operatorname{Aut}\left(\mathfrak{H} / \sim_{\pi}\right)$. 
We present a series of simple examples of finite covers; they illustrate different phenomena of finite covers on which we will comment later, referring back to these examples.

Example 2.19. Let $\vec{P}_{1} \cdot \omega$ be the directed graph which is an infinite union of directed edges. Then $\vec{P}_{1} \cdot \omega$ is a finite covering structure of $\mathbb{N}$, with $\pi$ being the projection to the second argument. Also note that $\operatorname{Aut}\left(\vec{P}_{1} \cdot \omega\right)$ is topologically isomorphic to $\operatorname{Sym}(\mathbb{N})$, and that $\vec{P}_{1} \cdot \omega$ and $(\mathbb{N} ; \neq)$ are bi-interpretable but not bi-definable.

Example 2.20. Let $K_{2} \cdot \omega$ be the graph which is an infinite union of undirected edges. Then $K_{2} \cdot \omega$ is a finite covering structure of $\mathbb{N}$, with $\pi$ being the projection to the second argument. Identifying the domain of $K_{2} \cdot \omega$ with $\{0,1\} \times \mathbb{N}$ so that $(u, n)$ is adjacent to $(v, m)$ if and only if $n=m$ and $u \neq v$, the automorphism group of $K_{2} \cdot \omega$ is the wreath product $\mathbb{Z}_{2} \prec \operatorname{Sym}(\omega)$ (see Section 2.3).

Example 2.21. Let $K_{2} \cdot \omega$ be the structure with domain $\{0,1\} \times \mathbb{N}$ from Example 2.20, and let $\mathfrak{A}$ be the expansion of $K_{2} \cdot \omega$ by the equivalence relation Eq defined by $\operatorname{Eq}((u, n),(v, m))$ if and only if $u=v$. Then $\mathfrak{A}$ is a finite covering structure of $\mathbb{N}$ with respect to the covering map $\pi$ that maps $(u, n)$ to $n$. Note that $\operatorname{Aut}(\mathfrak{V})$ is isomorphic (as an abstract group) to the direct product $\mathbb{Z}_{2} \times \operatorname{Sym}(\mathbb{N})$ (see Section 2.2 for direct products and other actions of direct products).

Example 2.22. Let $A:=\{0,1,2,3\} \times \mathbb{N}$, and let $\pi: A \rightarrow \mathbb{N}$ be the projection to the second argument. Let $\mathfrak{A}$ be the graph with vertex set $A$ such that $(u, b)$ is adjacent to $(v, c)$ if and only if

- $b=c$ and $u=v+1 \bmod 4$, or

- $b \neq c$ and $u=v \bmod 2$.

See Figure 1 for an illustration. Note that $\mathfrak{A}$ is a finite covering structure of $\mathbb{N}$ with respect to $\pi$. The automorphism group of $\mathfrak{A}$ equals $K H$, where

- $H=\left\{\alpha \in \operatorname{Sym}(A) \mid\right.$ if $\alpha(u, v)=\left(u^{\prime}, v^{\prime}\right)$, then $\left.u=u^{\prime}\right\}$ (i.e., $H$ is topologically isomorphic to $\operatorname{Sym}(\mathbb{N}))$, and

- $K=\left\{\alpha \in \prod_{i \in \mathbb{N}} \mathbb{Z}_{4} \mid\right.$ for all $\left.k, l \in \mathbb{N}, \alpha_{k} \mathbb{Z}_{2}=\alpha_{l} \mathbb{Z}_{2}\right\}$, where $\mathbb{Z}_{k}$ is the cyclic group acting on $\{0,1, \ldots, k-1\}$ and $\prod_{i \in \mathbb{N}} \mathbb{Z}_{4}$ is the direct product in its intransitive action on $A$ (see Section 2.2).

Example 2.23. Let $\mathfrak{B}$ be the countable structure which carries an equivalence relation Eq with three classes $R, S, T$ such that $|S|=|T|$, and a unary relation symbol denoting the class $R$. Let $A:=(\{0,1\} \times R) \cup(\{0\} \times(S \cup T))$. We define the 


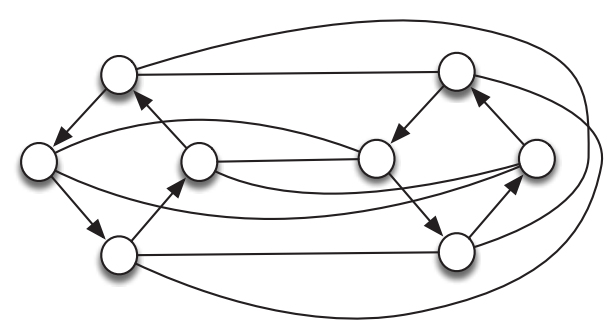

Figure 1. An illustration of the subgraph of the structure $\mathfrak{U}$ from Example 2.22 that is induced by 2 fibers.

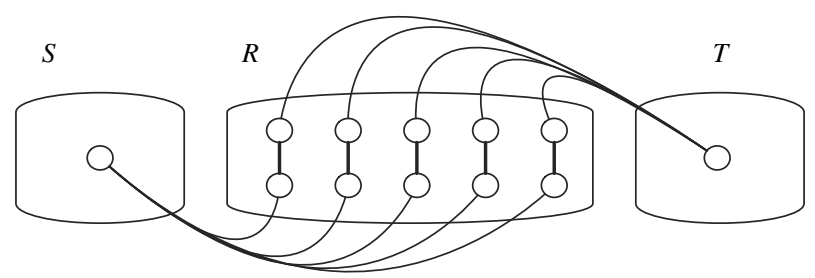

Figure 2. An illustration of a subgraph of the structure $\mathfrak{U}$ from Example 2.23 for the special case $|S|=|T|=1$.

structure $\mathfrak{U}$ with domain $A$ and the signature $\{E, F\}$, where $E$ and $F$ have arity two, and

- $E\left(\left(u_{1}, b_{1}\right),\left(u_{2}, b_{2}\right)\right)$ holds if and only if $\left(u_{1}=0, b_{1} \in R, b_{2} \in S\right)$ or $\left(u_{1}=1\right.$, $\left.b_{1} \in R, b_{2} \in T\right)$,

- $F\left(\left(u_{1}, b_{1}\right),\left(u_{2}, b_{2}\right)\right)$ holds if and only if $b_{1}=b_{2}$.

See Figure 2. Let $\pi: A \rightarrow B$ be the projection to the second argument. Then we have $\sim_{\pi}=F$, and $\pi$ is a finite covering. If $R, S, T$ are countably infinite, then $\sim_{\pi}=F=\Delta(\mathfrak{U})$. The automorphism group of $\mathfrak{U}$ is isomorphic to a semidirect product $\left(\operatorname{Sym}(R) \times \operatorname{Sym}(S)^{2}\right) \rtimes \mathbb{Z}_{2}$.

Definition 2.24. Let $\pi: \mathfrak{A} \rightarrow \mathfrak{B}$ be a finite covering map, $b \in B$, and $S:=\pi^{-1}(b)$.

- The fiber group of $\pi$ at $b$ is the group $\operatorname{Aut}(\mathfrak{U})_{\{S\}} \mid S$.

- The binding group of $\pi$ at $b$ is the group $\left.K\right|_{S}$, where $K$ is the kernel of $\mu_{\pi}$.

So the binding group at $b$ is a normal subgroup of the fiber group at $b$. If, for some $b \in B$, the fiber group and the binding group at $b$ are unequal, then $\pi$ is called twisted. Example 2.23 gives an example of a twisted finite cover; Examples 2.19, 2.20, 2.21, and 2.22 are not twisted. 
Remark 2.25. The following terminology is not needed for stating or proving our results, but we mention it for a better understanding of the examples of finite covers that we have already presented. Let $\pi: \mathfrak{A} \rightarrow \mathfrak{B}$ be a finite covering map, and let $B_{b}$ be the binding group at $b \in B$. Then $\pi$ is called free if the kernel of $\mu_{\pi}: \operatorname{Aut}(\mathfrak{Q}) \rightarrow \operatorname{Aut}(\mathfrak{B})$ equals $\prod_{b \in B} B_{b}$. Example 2.20, Example 2.19, Example 2.22, and Example 2.23 are free. Example 2.21 is an example of a finite cover which is not free: the binding group at each point is $\mathbb{Z}_{2}$ and equals the kernel of $\mu_{\pi}: \operatorname{Aut}(\mathfrak{U}) \rightarrow \operatorname{Aut}(\mathfrak{B})$, which is therefore not equal to $\prod_{b \in B} B_{b}=\mathbb{Z}_{2}^{\omega}$.

\subsection{Trivial finite covers}

There are two important notions of triviality for finite covers, intended to describe those finite covers that have an automorphism group which is smallest possible. This is important for our purposes since we will describe general finite covering structures in our class by describing them as certain first-order reducts of trivial finite covers, and as we will see, trivial covers are much easier to describe.

Definition 2.26. Let $\pi: \mathfrak{A} \rightarrow \mathfrak{B}$ be a finite covering map. We say that $\pi$ is

- a trivial cover if the kernel of $\mu_{\pi}: \operatorname{Aut}(\mathfrak{Q}) \rightarrow \operatorname{Aut}(\mathfrak{B})$ is trivial (only contains the identity permutation $\mathrm{id}_{A}$ ),

- a strongly trivial cover if all of its fiber groups are trivial.

A structure $\mathfrak{A}$ is called a (strongly) trivial covering structure of $\mathfrak{B}$ if there is a finite covering map $\pi: \mathfrak{U} \rightarrow \mathfrak{B}$ which is (strongly) trivial.

It is clear from the definition that $\pi$ is a trivial cover if and only if all of its binding groups are trivial. Hence, if $\pi$ is strongly trivial, then it is also trivial. Example 2.19 is an example of a strongly trivial finite covering. Example 2.23 is an example of a trivial finite covering which is not a strongly trivial finite covering. Examples 2.20, 2.21, and 2.22 are examples of nontrivial finite coverings.

Next we give a sufficient condition for a structure $\mathfrak{B}$ under which every trivial cover of $\mathfrak{B}$ is strongly trivial.

Lemma 2.27. Let $\mathfrak{B}$ be a structure such that the stabiliser $\operatorname{Aut}(\mathfrak{B})_{b}$ for every $b \in B$ has no nontrivial finite-index subgroups. Then every trivial cover of $\mathfrak{B}$ is strongly trivial.

Proof. Let $\pi: \mathfrak{A} \rightarrow \mathfrak{B}$ be a trivial finite cover. Then $\mu_{\pi}$ is an isomorphism between $\operatorname{Aut}(\mathfrak{Q})$ and $\operatorname{Aut}(\mathfrak{B})$. Let $b \in B$. We need to show that the fiber group of $\pi$ at $b$ is trivial. Put $S:=\pi^{-1}(b)$. Let us consider the mapping $\varphi$ : $\operatorname{Aut}(\mathfrak{B})_{b} \rightarrow \operatorname{Sym}(S)$ 
given by $\left.h \mapsto \mu_{\pi}^{-1}(h)\right|_{S}$. Then $\varphi$ is clearly a group homomorphism. Let $K$ be the kernel of this homomorphism. Then $K$ is a finite index subgroup of Aut $(\mathfrak{B})_{b}$, and thus, by our assumption, $K=\operatorname{Aut}(\mathfrak{B})_{b}$. That is, $\varphi$ is the trivial homomorphism, which means that the fiber group of $\pi$ at $b$ is trivial.

We now give an explicit description of strongly trivial covers.

Lemma 2.28. Let $\pi: \mathfrak{U} \rightarrow \mathfrak{B}$ be a strongly trivial covering map. Then, for each orbit $O$ of $\mathfrak{B}$, there exists a finite set $F_{O}$ and a mapping $\psi_{O}: \pi^{-1}(O) \rightarrow F_{O}$ such that,

- for every $w \in O$, the restriction of $\psi_{O}$ to $\pi^{-1}(w)$ is a bijection,

- $\psi_{O}(x)=\psi_{O}\left(\mu_{\pi}^{-1}(\beta)(x)\right)$ for all $x \in \pi^{-1}(O)$ and $\beta \in \operatorname{Aut}(\mathfrak{B})$.

Proof. Let us fix an element $b \in O$, and let $F_{O}:=\pi^{-1}(b)$. If $x \in \pi^{-1}(O)$, then there exists an automorphism $g$ of $\mathfrak{B}$ such that $g(\pi(x))=b$. Let us define $\psi_{O}(x)$ to be $\mu_{\pi}^{-1}(g)(x)$. We claim that $\psi_{O}(x) \in F_{O}$ and that its value is well-defined (i.e., it does not depend on our particular choice of $g$ ). The first claim is clear since, by definition,

$$
\pi\left(\mu_{\pi}^{-1}(g)(x)\right)=\mu_{\pi}\left(\mu_{\pi}^{-1}(g)\right)(\pi(x))=g(\pi(x))=b ;
$$

thus $\mu_{\pi}^{-1}(g)(x) \in \pi^{-1}(b)=F_{O}$.

In order to show the second claim, we need to show that if $h \in \operatorname{Aut}(\mathfrak{B})$ is such that $h(\pi(x))=g(\pi(x))=b$, then

$$
\mu_{\pi}^{-1}(g)(x)=\mu_{\pi}^{-1}(h)(x) .
$$

Since $\left(h^{-1} g\right)(\pi(x))=\pi(x)$, it follows that $\left.\left(\mu_{\pi}^{-1}\left(h^{-1} g\right)\right)\right|_{\pi(x)}$ is in the fiber group at $\pi(x)$. Since $\pi$ is strongly trivial, this group is trivial, and hence

$$
\mu_{\pi}^{-1}\left(h^{-1} g\right)(x)=x .
$$

This implies that

$$
\mu_{\pi}^{-1}(h)(x)=\left(\mu_{\pi}^{-1}(h) \mu_{\pi}^{-1}\left(h^{-1} g\right)\right)(x)=\mu_{\pi}^{-1}(g)(x) .
$$

Now the first item follows from the fact that if $w \in O$ is such that $g(w)=b$, then $g$ defines a bijection between $\pi^{-1}(w)$ and $\pi^{-1}(b)$. As for the second item, let $x \in \pi^{-1}(O)$, and let $g \in \operatorname{Aut}(\mathfrak{B})$ be such that $g(\pi(x))=b$. If $\beta \in \operatorname{Aut}(\mathfrak{B})$, then

$$
\left(g \beta^{-1}\right)\left(\pi\left(\mu_{\pi}^{-1}(\beta)(x)\right)\right)=\left(g \beta^{-1}\right)(\beta(\pi(x)))=g(\pi(x))=b,
$$

and thus

$$
\psi_{O}\left(\mu_{\pi}^{-1}(\beta)(x)\right)=\left(\mu_{\pi}^{-1}\left(g \beta^{-1}\right)\right)\left(\mu_{\pi}^{-1}(\beta)(x)\right)=\mu_{\pi}^{-1}(g)(x)=\psi_{O}(x) .
$$


Remark 2.29. Let the sets $F_{O}$ and the maps $\psi_{O}$ be defined as in Lemma 2.28 for each orbit $O$ of $\operatorname{Aut}(\mathfrak{B})$. Then there is a natural bijection between $A$ and $\bigcup_{O}\left(F_{O} \times O\right)$ defined as $x \mapsto\left(\psi_{O}(x), \pi(x)\right)$, where $O$ is the orbit of $\operatorname{Aut}(\mathfrak{B})$ containing $\pi(x)$. If we identify each element of $A$ with its image under this bijection, then $\operatorname{Aut}(\mathfrak{H})$ consists of those permutations that fix the first coordinate of each element and that act as an automorphism of $\mathfrak{B}$ on the second coordinate.

\subsection{Covering reducts}

Let $\mathfrak{A}$ and $\mathfrak{B}$ be structures, and let $\pi: \mathfrak{A} \rightarrow \mathfrak{B}$ be a finite covering map. A firstorder reduct $\mathfrak{E}$ of $\mathfrak{U}$ is a covering reduct of $\mathfrak{U}$ with respect to $\pi$ (and $\mathfrak{A}$ is called $a$ covering expansion of $\mathfrak{C}$ with respect to $\pi$; see [39]) if every $\alpha \in \operatorname{Aut}(\mathfrak{C})$ preserves $\sim_{\pi}$ and $\mu_{\pi}(\alpha) \in \operatorname{Aut}(\mathfrak{B})$.

Remark 2.30. We do not need but mention that every finite cover $\pi: \mathfrak{A} \rightarrow \mathfrak{B}$ is a covering expansion of a free finite covering structure of $\mathfrak{B}$ with respect to $\pi$ ([39, Lemma 2.1.3]).

Definition 2.31. Let $\pi: \mathfrak{A} \rightarrow \mathfrak{B}$ be a finite covering map.

- If $\mathfrak{A}$ is a covering reduct of a trivial covering structure of $\mathfrak{B}$ with respect to $\pi$, then $\pi$ is called a split cover of $\mathfrak{B}$ [39] (in this case, we also say that $\pi$ is split).

- If $\mathfrak{A}$ is a covering reduct of a strongly trivial covering of $\mathfrak{B}$ with respect to $\pi$, then $\pi$ is called a strongly split cover of $\mathfrak{B}$ [39].

Equivalently (this motivates the terminology; see [39]), a finite cover $\pi: \mathfrak{O} \rightarrow \mathfrak{B}$ is split if the kernel $K$ of $\mu_{\pi}: \operatorname{Aut}(\mathfrak{H}) \rightarrow \operatorname{Aut}(\mathfrak{B})$ has a closed complement in $\operatorname{Aut}(\mathfrak{U})$, i.e., there is a closed subgroup $H$ of $\operatorname{Aut}(\mathfrak{U})$ such that $K H=\operatorname{Aut}(\mathfrak{U})$ and $K \cap H=\{1\}$ (so that $\operatorname{Aut}(\mathfrak{N})$ is isomorphic to the semidirect product $K \rtimes H$ ). Examples 2.19, 2.202 .21 , and 2.22 are examples of split covers of $\mathbb{N}$. For a nonexample, see, e.g., [40]. Example 2.23, in the case that $|S|=|T|=1$, is an example of a finite split cover of a structure in $U$ which is not strongly split.

\subsection{Operations on classes of structures}

Let $\mathfrak{U}$ be a structure, and let $\mathfrak{B}$ be a first-order reduct of $\mathfrak{A}$. Then we say that $\mathfrak{B}$ is a finite index (first-order) reduct of $\mathfrak{U}$ if and only if the index [Aut( $\mathfrak{B})$ : Aut( $\mathfrak{U})$ ] is finite. We define the following operations on classes of structures.

Definition 2.32. Let $\mathfrak{U}$ be a countable $\omega$-categorical structure. Then

- $C(\mathfrak{U})$ is the class of structures which are interdefinable with an expansion of $\mathfrak{U}$ with finitely many constants, 
- $M(\mathfrak{U})$ is the class of structures that are interdefinable with the (up to isomorphism unique $[8,12])$ model-complete core of $\mathfrak{A}$,

- $R(\mathfrak{U})$ is the class of first-order reducts of $\mathfrak{A}$,

- $R^{<\infty}(\mathfrak{V})$ is the class of finite index first-order reducts of $\mathfrak{A}$,

- $F(\mathfrak{U})$ is the class of finite covering structures of $\mathfrak{A}$.

If $\mathcal{C}$ is a class of structures and $\Phi$ is one of the operators above, then we use the notation $\Phi(\mathcal{C})$ for the union of the classes $\Phi(\mathfrak{H})$ such that $\mathfrak{U} \in \mathcal{C}$.

Proposition 2.33. The following identities hold.

(1) $C \circ C=C$,

(2) $M \circ M=M$,

(3) $R \circ R=R$,

(4) $R^{<\infty} \circ R^{<\infty}=R^{<\infty}$,

(5) $F \circ F=F$,

(6) $C\left(\mathcal{U}_{\mathrm{nf}}\right)=\mathcal{U}^{*}$,

(7) $R(\mathcal{U})=R\left(\mathcal{U}^{*}\right)$,

(8) $\mathcal{K}_{\exp }=R\left(\mathcal{K}_{\text {exp }}\right)$,

(9) $\mathcal{K}_{\exp +}=R\left(\mathcal{K}_{\exp +}\right)$.

Proof. Straightforward from the definitions.

We will show that $\mathcal{K}_{\exp }=R(\mathcal{U})$ and $\mathcal{K}_{\exp +}=(F \circ R)(\mathcal{U})=(R \circ F)(\mathcal{U})$, and we will give several equivalent descriptions of these classes in Section 8. We also prove Thomas' conjecture for each structure in $\mathcal{K}_{\exp +}$ (Theorem 6.37).

\section{Reducts of unary structures}

In this section, we characterise first-order reducts of unary structures in terms of their automorphism groups, and in particular prove Thomas' conjecture for the class $R(U)$. We mention that the finite-domain constraint satisfaction tractability conjecture has been shown for all structures in $R(U)$ (see [15]).

Lemma 3.1. Let $\mathfrak{A}$ be a structure. Then $\mathfrak{A} \in \mathcal{U}$ if and only if there are finitely many sets $O_{1}, \ldots, O_{k}$ such that $\operatorname{Aut}(\mathfrak{H})=\prod_{i=1}^{k} \operatorname{Sym}\left(O_{i}\right)$. 
Proof. Suppose that $\mathfrak{A} \in \mathcal{U}$. Then $\mathfrak{A}$ is interdefinable with a unary structure $\mathfrak{U}^{\prime}$; let $O_{1}, \ldots, O_{k}$ be the minimal non-empty intersections of predicates from $\mathfrak{A}^{\prime}$; clearly, these sets partition $A$. The containment $\operatorname{Aut}(\mathfrak{U}) \subseteq \prod_{i=1}^{k} \operatorname{Sym}\left(O_{i}\right)$ is clear since every automorphism of $\mathfrak{A}$ is an automorphism of $\mathfrak{U}^{\prime}$ and hence preserves the sets $O_{1}, \ldots, O_{k}$. For the reverse containment, let $\alpha \in \operatorname{Sym}(A)$ be such that $\alpha\left(O_{i}\right)=O_{i}$ for all $i \leq k$. Since $\operatorname{Aut}(\mathfrak{U})=\operatorname{Aut}\left(\mathfrak{U}^{\prime}\right)$, we need to show that $\alpha$ preserves all (unary) relations $U$ of $\mathfrak{U}^{\prime}$. Let $x \in U$, and let $i \leq k$ be such that $x \in O_{i}$. Since $U$ must be a union of orbits of $\operatorname{Aut}\left(\mathfrak{Q}^{\prime}\right)$, we have that $\alpha(x) \in O_{i}$ also lies in $U$.

Conversely, suppose that $\operatorname{Aut}(\mathfrak{U})=\prod_{i=1}^{k} \operatorname{Sym}\left(O_{i}\right)$. Then $\mathfrak{A}$ is first-order interdefinable with the unary structure $\mathfrak{U}=\left(X ; O_{1}, \ldots, O_{k}\right)$.

The following statement is an easy consequence of Lemmas 3.1 and 2.14.

Corollary 3.2. Let $\mathfrak{U} \in \mathcal{U}$. Then the $\nabla(\mathfrak{U})$-classes are the infinite orbits of $\operatorname{Aut}(\mathfrak{U})$ and the singleton orbits.

Lemma 3.3. Let $\mathfrak{U} \in R(\mathcal{U})$, and let $C_{1}, \ldots, C_{k}$ be the $\nabla(\mathfrak{U})$-classes. Then

$$
\prod_{i=1}^{k} \operatorname{Sym}\left(C_{i}\right) \subseteq \operatorname{Aut}(\mathfrak{U}) .
$$

Proof. Let $\mathfrak{A}$ be a first-order reduct of a structure $\mathfrak{B} \in \mathcal{U}$. Let $O_{1}, \ldots, O_{l}$ be the orbits of $\mathfrak{B}$. Then $\operatorname{Aut}(\mathfrak{B})=\prod_{i=1}^{l} \operatorname{Sym}\left(O_{i}\right)$ by Lemma 3.1. Let us define the binary relation $R$ on $A$ so that $x R y$ if and only if $x=y$ or the transposition ( $x y$ ) is contained in $\operatorname{Aut}(\mathfrak{Q})$. Then it is easy to see that $R$ is a congruence of $\operatorname{Aut}(\mathfrak{U})$. On the other hand, $\operatorname{Aut}(\mathfrak{X}) \supseteq \operatorname{Aut}(\mathfrak{B})=\prod_{i=1}^{l} \operatorname{Sym}\left(O_{i}\right)$ implies that each class of $R$ is the union of some of the orbits of $\operatorname{Aut}(\mathfrak{B})$. In particular, $R$ has finitely many classes, and so, by definition, the congruence $\nabla(\mathfrak{H})$ is finer that $R$. This means that, for all $x, y \in C_{i}$, the transposition $(x y)$ is contained in Aut( $\left.\mathfrak{A}\right)$. Therefore, $\prod_{i=1}^{k} \operatorname{Sym}\left(C_{i}\right) \subseteq \operatorname{Aut}(\mathfrak{U})$ since $\operatorname{Aut}(\mathfrak{U})$ is closed.

Corollary 3.4. $R(U)=R^{<\infty}(U)=R^{<\infty}\left(U^{*}\right)$.

Proof. The containments " $\supseteq$ " are obvious. Let $\mathfrak{U} \in R(\mathcal{U})$. Let $C_{1}, \ldots, C_{k}$ be the classes of $\nabla(\mathfrak{H})$. Then the group $\operatorname{Aut}(\mathfrak{N})$ acts on the set $\left\{C_{1}, \ldots, C_{k}\right\}$. By Lemma 3.3, the kernel of this action is $\prod_{i=1}^{k} \operatorname{Sym}\left(C_{i}\right)$. In particular, the index of $\prod_{i=1}^{k} \operatorname{Sym}\left(C_{i}\right)$ in $\operatorname{Aut}(\mathfrak{2})$ is finite. On the other hand, $\prod_{i=1}^{k} \operatorname{Sym}\left(C_{i}\right)$ is the automorphism group of a unary $\omega$-categorical structure with orbits $C_{1}, \ldots, C_{k}$. We also know from Lemma 2.14 that each class $C_{i}$ is either a singleton or infinite. Therefore, $\mathfrak{A} \in R^{<\infty}\left(U^{*}\right)$. 
The following has been shown in [15, Proposition 6.8]; the proof we present here is simpler.

Corollary 3.5. Let $\mathfrak{A} \in R(\mathcal{U})$. Then there exists an expansion of $\mathfrak{A}$ with finitely many constants which is in $\mathcal{U}^{*}$.

Proof. Let $C_{1}, \ldots, C_{k}$ be the classes of $\nabla(\mathfrak{U})$, and let us choose elements $c_{i} \in C_{i}$. We claim that the structure $\left(\mathfrak{A}, c_{1}, \ldots, c_{k}\right)$ is in $U^{*}$. By Lemma 2.14 , we know that each class $C_{i}$ is either a singleton or infinite. Without loss of generality, we can assume that $C_{i}=\left\{c_{i}\right\}$ for $i=1, \ldots, l$ and $C_{j}$ is infinite for $j>l$. We claim that

$$
\operatorname{Aut}\left(\mathfrak{A} ; c_{1}, \ldots, c_{k}\right)=\prod_{i=1}^{k} \operatorname{id}\left(\left\{c_{i}\right\}\right) \times \prod_{i=l+1}^{k} \operatorname{Sym}\left(C_{i} \backslash\left\{c_{i}\right\}\right) .
$$

Then Lemma 3.1 implies that $\left(\mathfrak{U}, c_{1}, \ldots, c_{k}\right) \in U^{*}$. To prove the claim, first recall from Lemma 3.3 that $\prod_{i=1}^{k} \operatorname{Sym}\left(C_{i}\right) \subseteq \operatorname{Aut}(\mathfrak{U})$, and hence

$$
\prod_{i=1}^{k} \operatorname{Sym}\left(C_{i}\right)_{\left\{c_{1}, \ldots, c_{k}\right\}} \subseteq \operatorname{Aut}(\mathfrak{W})_{\left\{c_{1}, \ldots, c_{k}\right\}} \cdot
$$

Since every automorphism of $\mathscr{U}$ that fixes $c_{1}, \ldots, c_{k}$ must also preserve the sets $C_{1}, \ldots, C_{k}$, we in fact have equality $\prod_{i=1}^{k} \operatorname{Sym}\left(C_{i}\right)_{\left\{c_{1}, \ldots, c_{k}\right\}}=\operatorname{Aut}(\mathfrak{H})_{\left\{c_{1}, \ldots, c_{k}\right\}}$. Thus,

$$
\begin{aligned}
\operatorname{Aut}\left(\mathfrak{U} ; c_{1}, \ldots, c_{k}\right) & =\operatorname{Aut}(\mathfrak{H})_{\left\{c_{1}, \ldots, c_{k}\right\}} \\
& =\prod_{i=1}^{k} \operatorname{Sym}\left(C_{i}\right)_{\left\{c_{1}, \ldots, c_{k}\right\}} \\
& =\prod_{i=1}^{k} \operatorname{id}\left(\left\{c_{i}\right\}\right) \times \prod_{i=l+1}^{k} \operatorname{Sym}\left(C_{i} \backslash\left\{c_{i}\right\}\right) .
\end{aligned}
$$

Lemma 3.6. Let $\mathfrak{A} \in R(\mathcal{U})$, and let $C_{1}, \ldots, C_{k}$ be the $\nabla(\mathfrak{N})$-classes. Then we have $\operatorname{Aut}(\mathfrak{U})=\prod_{i=1}^{k} \operatorname{Sym}\left(C_{i}\right) \rtimes A$, where $A$ is a subgroup of $\operatorname{Aut}(\mathfrak{N})$ acting faithfully on $\left\{C_{1}, \ldots, C_{k}\right\}$.

Proof. Recall from Lemma 2.14 that every class of $\nabla(\mathfrak{Q})$ is either infinite or a singleton. We can assume that $C_{1}, \ldots, C_{l}$ are infinite and, for every $j \in\{l+1, \ldots, k\}$, there exists $c_{j} \in A$ such that $C_{j}=\left\{c_{j}\right\}$. Let $e_{i}: C_{i} \rightarrow \mathbb{N}$, for $1 \leq j \leq l$, be bijections, and let $e=\bigcup_{i \in\{1, \ldots, l\}} e_{i}$. Then it is easy to see that, for every $\alpha \in \operatorname{Aut}(\mathfrak{U})$, there exists $\tilde{\alpha} \in \operatorname{Sym}(A)$ such that

(1) $\alpha$ and $\tilde{\alpha}$ have the same action on the set $\left\{C_{1}, \ldots, C_{k}\right\}$,

(2) $e(\tilde{\alpha}(x))=e(x)$ for every $x \in \bigcup_{i \in\{1, \ldots, l\}} C_{i}$. 
The permutation $\alpha^{-1} \tilde{\alpha}$ fixes every class of $\nabla(\mathfrak{H})$. Since

$$
N:=\prod_{i=1}^{k} \operatorname{Sym}\left(C_{i}\right) \subseteq \operatorname{Aut}(\mathfrak{U})
$$

by Lemma 3.3, it follows that $\alpha^{-1} \tilde{\alpha} \in \operatorname{Aut}(\mathfrak{H})$ and thus $\tilde{\alpha} \in \operatorname{Aut}(\mathfrak{U})$. Let

$$
A:=\{\tilde{\alpha} \mid \alpha \in \operatorname{Aut}(\mathfrak{U})\} .
$$

Then $A \subseteq \operatorname{Aut}(\mathfrak{U})$ is a subgroup of $G$ which acts faithfully on $\left\{C_{1}, \ldots, C_{k}\right\}$. Then it is also clear that $N$ is a normal subgroup of $\operatorname{Aut}(\mathfrak{O})$ since it is the kernel of the action of $\operatorname{Aut}(\mathfrak{U})$ on $\left\{C_{1}, \ldots, C_{k}\right\}$. Therefore, $\operatorname{Aut}(\mathfrak{U})$ can be written as a semidirect product, $\operatorname{Aut}(\mathfrak{U})=\prod_{i=1}^{k} \operatorname{Sym}\left(C_{i}\right) \rtimes A$.

Corollary 3.7. Let $\mathfrak{U} \in R(\mathcal{U})$ be with no finite orbits. Then Aut $(\mathfrak{H})$ is isomorphic to the wreath product $\operatorname{Sym}(\mathbb{N})$ ? A for some permutation group $A$ on a finite set.

Proof. Let $C_{1}, \ldots, C_{k}$ be the classes of $\nabla(\mathfrak{U})$. Without loss of generality, we can assume that $C_{i}=\{(i, n) \mid n \in \mathbb{N}\}$. Let $A$ be the image of the action of Aut( $(\mathfrak{U})$ on the set $\left\{C_{1}, \ldots, C_{k}\right\}$. Then if we use the bijections $e_{i}:(i, n) \mapsto n$ in the proof of Lemma 3.6, the statement of the corollary follows.

Lemma 3.8. Suppose that $\mathfrak{H}, \mathfrak{B} \in R(\mathcal{U})$ have the same domain. If $\nabla(\mathfrak{H})=\nabla(\mathfrak{B})$ and the actions of the groups $\operatorname{Aut}(\mathfrak{U})$ and $\operatorname{Aut}(\mathfrak{B})$ on the $\nabla(\mathfrak{U})$-classes are the same, then $\mathfrak{A}$ and $\mathfrak{B}$ are interdefinable.

Proof. By the $\omega$-categoricity of $\mathfrak{A}$ and $\mathfrak{B}$, it is enough to show that

$$
\operatorname{Aut}(\mathfrak{U})=\operatorname{Aut}(\mathfrak{B}) .
$$

Let $C_{1}, \ldots, C_{k}$ be the classes of $\nabla(\mathfrak{U})=\nabla(\mathfrak{B})$. Lemma 3.3 shows that

$$
\prod_{i=1}^{k} \operatorname{Sym}\left(C_{i}\right) \subseteq \operatorname{Aut}(\mathfrak{U}) .
$$

Now let $\beta \in \operatorname{Aut}(\mathfrak{B})$. By our assumption about the action of $\operatorname{Aut}(\mathfrak{U})$ and $\operatorname{Aut}(\mathfrak{B})$ on the $\nabla(\mathfrak{U})$-classes, there is a permutation $\alpha \in \operatorname{Aut}(\mathfrak{U})$ such that $\beta \alpha^{-1}$ fixes each class $C_{i}$. Then $\beta \alpha^{-1} \in \prod_{i=1}^{k} \operatorname{Sym}\left(C_{i}\right) \subseteq \operatorname{Aut}(\mathfrak{H})$, and so $\beta \in \operatorname{Aut}(\mathfrak{U})$. Therefore, $\operatorname{Aut}(\mathfrak{B}) \subseteq \operatorname{Aut}(\mathfrak{Q})$. Analogously, $\operatorname{Aut}(\mathfrak{U}) \subseteq \operatorname{Aut}(\mathfrak{B})$.

Corollary 3.9. Every structure in $\mathcal{U}$ has finitely many first-order reducts. 
Proof. Let $\mathfrak{A} \in \mathcal{U}$. Then, by Lemma 3.1, $\operatorname{Aut}(\mathfrak{U})=\prod_{i=1}^{k} \operatorname{Sym}\left(O_{i}\right)$. If $\mathfrak{B}$ is a firstorder reduct of $\mathfrak{A}$, then $\nabla(\mathfrak{B})$ is a union of orbits of $\mathfrak{A}$. This means that there are finitely many choices for the relation $\nabla(\mathfrak{B})$. If the relation $\nabla(\mathfrak{B})$ is fixed, then there are finitely many possible actions of $\operatorname{Aut}(\mathfrak{B})$ on the classes of $\nabla(\mathfrak{B})$. By Lemma 3.8, it follows that $\nabla(\mathfrak{B})$ and the action of $\operatorname{Aut}(\mathfrak{B})$ on the classes of $\nabla(\mathfrak{B})$ already determine the structure $\mathfrak{B}$ up to interdefinability. Therefore, $\mathfrak{O}$ has finitely many first-order reducts.

We also obtain an equivalent description of first-order reducts of unary structures in terms of their automorphism groups.

Corollary 3.10. A structure $\mathfrak{A}$ is in $R(\mathcal{U})$ if and only if $\prod_{i=1}^{k} \operatorname{Sym}\left(C_{i}\right) \subseteq \operatorname{Aut}(\mathfrak{U})$ for some partition of $A$ into classes $C_{1}, \ldots, C_{k}$.

Proof. One direction has been shown in Lemma 3.3. For the converse implication, suppose that $\operatorname{Aut}(\mathfrak{U})$ contains $\prod_{i=1}^{k} \operatorname{Sym}\left(C_{i}\right)$ for some partition of $A$ into classes $C_{1}, \ldots, C_{k}$. Note that $\operatorname{Aut}\left(A ; C_{1}, \ldots, C_{k}\right)=\prod_{i=1}^{k} \operatorname{Sym}\left(C_{i}\right)$ (see Lemma 3.1) and that $\mathfrak{U}$ is a first-order reduct of $\left(A ; C_{1}, \ldots, C_{k}\right)$. Hence, $\mathfrak{A} \in R(\mathcal{U})$.

\section{Finite coverings of unary structures}

In this section, we classify the finite coverings of unary structures. First we make the following observation.

Lemma 4.1. $F(U)=F\left(U^{*}\right)$.

Proof. The containment " $\supseteq$ " is trivial. In order to show the other direction, it is enough to show that $\mathcal{U} \subseteq F\left(\mathcal{U}^{*}\right)$ since $F \circ F=F$. So let $\mathfrak{A} \in \mathcal{U}$, and let $F$ be the union of the finite orbits of $\mathfrak{A}$. Then $F$ is finite. Let us consider the unary structure $\mathfrak{B}$ whose domain is $B:=A \backslash F \cup\{x\}$ for any $x \notin A$, and whose relations are the infinite orbits of $\mathfrak{A}$ and $\{x\}$. Then $\mathfrak{B} \in \mathcal{U}^{*}$. Let $\pi: \mathfrak{U} \rightarrow \mathfrak{B}$ be defined as $\pi(y)=x$ if $x \in F$, and $\pi(y)=y$ otherwise. Then it is easy to see that $\pi$ is a finite covering map, and hence $\mathfrak{A} \in F\left(\mathcal{U}^{*}\right)$.

The following theorem summarises the results from Section 4.1 and Section 4.2.

Theorem 4.2. Let $\mathfrak{B} \in \mathcal{U}^{*}$, and let $\pi: \mathfrak{A} \rightarrow \mathfrak{B}$ be a finite covering map. Then $\mathfrak{A}$ has finitely many covering reducts with respect to $\pi$.

Proof. Proposition 4.9 shows that $\pi$ is strongly split. The statement then follows from Corollary 4.28 . 


\subsection{Finite covers of unary structures split}

The following series of lemmas is needed to show that every finite covering map of a structure $\mathfrak{B} \in U^{*}$ is strongly split (Proposition 4.9). Throughout this subsection, let $\mathfrak{B} \in \mathcal{U}^{*}$, and let $\pi: \mathfrak{A} \rightarrow \mathfrak{B}$ be a finite covering map.

Remark 4.3. Observe that $\mathfrak{B}$ satisfies the condition of Lemma 2.27, i.e., Aut $(\mathfrak{B})_{x}$ has no finite index subgroup for any $x \in B$. By Lemma 2.27, this implies that every trivial finite cover of $\mathfrak{B}$ is strongly trivial, and hence every split cover of $\mathfrak{B}$ is strongly split.

Remark 4.4. When $\mathfrak{B}$ is taken from $\mathcal{U}$ instead of $\mathcal{U}^{*}$, then there are split covers of $\mathfrak{B}$ that are not strongly split, as illustrated by Example 2.23 if $|S|=|T|=1$.

Lemma 4.5. Let $F$ be a finite subset of an infinite orbit $O$ of $\mathfrak{B}$. If $|F|$ is large enough, then there exists an automorphism $\alpha$ of $\mathfrak{A}$ such that

(1) $\alpha(x)=x$ for all $x \in E:=A \backslash \pi^{-1}(F)$,

(2) $\left.\mu_{\pi}(\alpha)\right|_{F}$ is nontrivial.

Proof. Let $k$ be the maximum of the sizes of the fibers of $\pi$, and let $p>k$ be a prime number. We claim that if $|F| \geq p$, then there is an automorphism $\alpha$ of $\mathfrak{U}$ satisfying conditions (1) and (2).

Let $u_{1}, \ldots, u_{p} \in F$ be distinct elements. Then the $p$-cycle $\left(u_{1} u_{2} \ldots u_{p}\right)$ is contained in Aut( $(\mathfrak{B})$ by Lemma 3.1. By the definition of finite covering maps, there exists $\beta \in \operatorname{Aut}(\mathfrak{U})$ such that $\mu_{\pi}(\beta)=\left(u_{1} \ldots u_{p}\right)$. Now let $\alpha:=\beta^{k !} \in \operatorname{Aut}(\mathfrak{U})$. Then $\left.\mu_{\pi}(\alpha)\right|_{F}$ is again a $p$-cycle and hence nontrivial. On the other hand, if $u \in B \backslash F$ and $U:=\pi^{-1}(u)$, then $\beta(U)=U$, and $\left.\alpha\right|_{U}=\left.\beta^{k !}\right|_{U}=\operatorname{id}_{U}$ since $|U| \leq k$. This means that $\left.\alpha\right|_{E}=\mathrm{id}_{E}$. Therefore, $\alpha \in \operatorname{Aut}(\mathfrak{U})$ satisfies conditions (1) and (2), which proves the lemma.

Recall that, for any finite set $F$ of cardinality at least 5 , the alternating group $\operatorname{Alt}(F)$ is the only nontrivial proper normal subgroup of $\operatorname{Sym}(F)$ (see, e.g., [36, Chapter 8.1]).

Lemma 4.6. Let $F$ be a finite subset of an infinite orbit $O$ of $\mathfrak{B}$. If $|F|$ is large enough, then for any pairwise distinct $u_{1}, u_{2}, u_{3}, u_{4} \in F$, there exists an automorphism $\alpha$ of $\mathfrak{U}$ such that

(1) $\alpha(x)=x$ for all $x \in E:=A \backslash \pi^{-1}(F)$,

(2) $\left.\mu_{\pi}(\alpha)\right|_{F}=\left(u_{1} u_{2}\right)\left(u_{3} u_{4}\right)$. 
Proof. Let $K:=\left\{\left.\mu_{\pi}(\gamma)\right|_{F} \in \operatorname{Sym}(F) \mid \gamma \in \operatorname{Aut}(\mathfrak{U})_{E}\right\}$. We claim that $K$ is a normal subgroup of $\operatorname{Sym}(F)$. It is clear that $K$ is a subgroup of $\operatorname{Sym}(F)$. Let $\alpha \in K$ and $\beta \in \operatorname{Sym}(F)$. We need to show that $\beta \alpha \beta^{-1} \in K$. By the definition of $K$, there exists $\gamma \in \operatorname{Aut}(\mathfrak{U})_{E}$ so that $\left.\mu_{\pi}(\gamma)\right|_{F}=\alpha$. By Lemma 3.1, there exists $\delta \in \operatorname{Aut}(\mathfrak{B})$ so that $\left.\delta\right|_{F}=\beta$. By the definition of finite covers, there exists $\eta \in \operatorname{Aut}(\mathfrak{U})$ such that $\mu_{\pi}(\eta)=\delta$. Let $\gamma^{\prime}=\eta \gamma \eta^{-1} \in \operatorname{Aut}(\mathfrak{U})$. Then on can check that $\gamma^{\prime}(x)=x$ for all $x \in E$ and

$$
\left.\mu_{\pi}\left(\gamma^{\prime}\right)\right|_{F}=\left.\left(\mu_{\pi}(\eta) \mu_{\pi}(\gamma) \mu_{\pi}(\eta)^{-1}\right)\right|_{F}=\left.\left.\left.\delta\right|_{F} \mu_{\pi}(\gamma)\right|_{F} \delta^{-1}\right|_{F}=\beta \alpha \beta^{-1} .
$$

We obtained that $K \triangleleft \operatorname{Sym}(F)$. By Lemma 4.5, we know that if $F$ is large enough, then $K$ is nontrivial. Therefore, if $F$ is large enough, then $K \geq \operatorname{Alt}(F)$, and then the statement of the lemma follows.

Lemma 4.7. Let $O$ be an infinite orbit of $\mathfrak{B}$. Then, for all distinct $v_{1}, v_{2} \in O$, there exists an $\alpha \in \operatorname{Aut}(\mathfrak{U})$ such that

(1) $\alpha(x)=x$ for all $x \in A \backslash \pi^{-1}\left(\left\{v_{1}, v_{2}\right\}\right)$,

(2) $\mu_{\pi}(\alpha)=\left(v_{1} v_{2}\right)$.

Proof. Let $v_{1}, v_{2} \in O$, and let us choose a finite subset $F$ of $O$ which contains the elements $v_{1}$ and $v_{2}$, which is large enough so that we can apply Lemma 4.6 for $F$. Choose $u_{3}, u_{4} \in F$ such that $u_{1}:=v_{1}, u_{2}:=v_{2}, u_{3}, u_{4}$ are pairwise distinct. Let $\alpha \in \operatorname{Aut}(\mathfrak{U})$ be as in Lemma 4.6. For each $i \in \mathbb{N}$, choose $\gamma_{i} \in \operatorname{Aut}(\mathfrak{H})$ so that

$$
\begin{aligned}
\mu_{\pi}\left(\gamma_{i}\right)\left(v_{1}\right) & =v_{2}, \\
\mu_{\pi}\left(\gamma_{i}\right)\left(v_{2}\right) & =v_{1}, \\
\mu_{\pi}\left(\gamma_{i}\right)(F) \cap \mu_{\pi}\left(\gamma_{j}\right)(F) & =\left\{v_{1}, v_{2}\right\} \quad \text { for all } i \neq j .
\end{aligned}
$$

By Lemma 3.1, it follows that such $\gamma_{i}$ 's exist. Let $\beta_{i}:=\gamma_{i} \alpha \gamma_{i}^{-1} \in \operatorname{Aut}(\mathfrak{H})$. Then $\mu_{\pi}\left(\beta_{i}\right)\left(v_{1}\right)=v_{2}, \mu_{\pi}\left(\beta_{i}\right)\left(v_{2}\right)=v_{1}$, and for all $x \in S:=\pi^{-1}\left(\left\{v_{1}, v_{2}\right\}\right)$, we have $\beta_{i}(x)=x$ if $i$ is large enough. Since there are finitely many possible actions of $\beta_{i}$ on the finite set $S$, there is a subsequence $\left(\beta_{l(i)}\right)_{i}$ of $\left(\beta_{i}\right)_{i}$ so that $\beta_{l(i)}\left|S=\beta_{l(j)}\right| S$ for all $i, j \in \mathbb{N}$. Then the sequence $\beta_{l(i)}$ converges to a permutation $\beta$ for which $\beta(x)=x$ for all $x \in A \backslash S$ and $\pi(\beta)=\pi\left(\beta_{l(1)}\right)=\left(v_{1} v_{2}\right)$. Since Aut( $(2)$ is closed, it follows that $\beta \in \operatorname{Aut}(\mathfrak{Q})$, which finishes the proof of the lemma.

Lemma 4.8. Let $\mathcal{O}$ be the set of orbits of $\mathfrak{B}$. Then, for each $O \in \mathcal{O}$, there exists a finite set $F_{O}$ and a mapping $\psi_{O}: \pi^{-1}(O) \rightarrow F_{O}$ such that $\left.\psi_{O}\right|_{\pi^{-1}(y)}$ is bijective for every $y \in O$, and $\operatorname{Aut}(\mathfrak{U})$ contains every $\alpha \in \operatorname{Sym}(A)$ such that

(1) $\alpha$ preserves $\sim_{\pi}$, 
(2) $\mu_{\pi}(\alpha) \in \operatorname{Aut}(\mathfrak{B})$,

(3) $\psi_{O}(x)=\psi_{O}(\alpha(x))$ for every $O \in \mathcal{O}$ and $x \in \pi^{-1}(O)$.

Proof. If $O$ is finite, then $O=\{u\}$ for some $u \in B$ since $\mathfrak{B} \in \mathcal{U}^{*}$. In this case, let $\psi_{O}=\operatorname{id}_{\pi^{-1}(u)}$. If $O$ is infinite, then we define $\psi_{O}$ as follows. Let $u \in O$ be arbitrary.

- If $x \in \pi^{-1}(u)$, then set $\psi_{O}(x):=x$.

- If $x \in \pi^{-1}(O) \backslash \pi^{-1}(u)$, then by Lemma 4.7, there exists a permutation

$$
\left.\alpha \in \operatorname{Aut}(\mathfrak{U})\right|_{A \backslash \pi^{-1}(\{\pi(x), u\})}
$$

such that $\alpha\left(\pi^{-1}(\pi(x))\right)=\pi^{-1}(u)$. In particular, $\alpha$ defines a bijection between $\left.\pi^{-1}(\pi(x))\right)$ and $\pi^{-1}(u)$. Set $\psi_{O}(x):=\alpha(x)$.

We claim that these mappings satisfy the conditions of the lemma. Let $G$ be the permutation group of those $\gamma \in \operatorname{Sym}(B)$ for which there exists an automorphism $\alpha$ of $\mathfrak{U}$ with $\left(\mu_{\pi}(\alpha)\right)=\gamma$ and satisfying conditions (1)-(3) of the lemma. Then, since $\operatorname{Aut}(\mathfrak{B})=\prod_{O \in \mathcal{O}} \operatorname{Sym}(O)$, it is enough to show that $\left.\left(G_{B \backslash O}\right)\right|_{O}=\operatorname{Sym}(O)$ for all $O \in \mathcal{O}$. If $O$ is a singleton, then the claim is trivial, so we can assume that $O$ is infinite. It is easy to see that $G$ is closed. Thus, $\left(G_{B \backslash O}\right) \mid O$ is also closed. Hence, by Lemma 3.1, it is enough to show that $G$ contains for all $u_{1}, u_{2} \in O$ the transposition $\left(u_{1} u_{2}\right)$. For this, it is enough to show that $(u v) \in G$ for all $v \in O \backslash\{u\}$, where $u$ is the element of $O$ which is used in the definition of $\psi_{O}$. But this follows directly from the definition of the mapping $\psi_{O}$.

Proposition 4.9. Any finite covering map $\pi: \mathfrak{U} \rightarrow \mathfrak{B}$ for $\mathfrak{B} \in \mathcal{U}^{*}$ is strongly split.

Proof. Let $F_{O}$ and $\psi_{O}$ be defined as in Lemma 4.8 for each orbit $O$ of $\mathfrak{B}$. Let $F:=\bigsqcup_{O} F_{O}$ and $\psi:=\bigcup_{O} \psi_{O}$. Let $\mathfrak{X}^{\prime}$ be the expansion of $\mathfrak{U}$ obtained by adding to $\mathfrak{U}$ for each $x \in F$ the unary relation $\psi^{-1}(x)$. Then, by Lemma 4.8 , it follows that $\mu_{\pi}\left(\operatorname{Aut}\left(\mathfrak{U}^{\prime}\right)\right)=\operatorname{Aut}(\mathfrak{B})=\mu_{\pi}(\operatorname{Aut}(\mathfrak{U}))$. Thus, $\mathfrak{U}$ is a covering reduct of $\mathfrak{U}^{\prime}$. We claim that $\pi: \mathfrak{U} \mathfrak{X}^{\prime} \rightarrow \mathfrak{B}$ is a strongly trivial cover. This implies the statement of the proposition. By Remark 4.3, it is enough to show that the finite cover $\pi: \mathfrak{U} \mathfrak{\prime}^{\prime} \rightarrow \mathfrak{B}$ is trivial, i.e., that the kernel of the map $\mu_{\pi}$ is trivial. Let $\left.\alpha \in \operatorname{Aut}(\mathfrak{U})^{\prime}\right)$ be so that $\mu_{\pi}(\alpha)=\operatorname{id}_{B}$, and let $x \in A$. Then $x \sim_{\pi} \alpha(x)$ and $\psi(x)=\psi(\alpha(x))$. It follows from the definition that $\psi$ is injective on $[x]_{\pi}$. This implies that $x=\alpha(x)$ and hence that $\alpha=\operatorname{id}_{A}$. Therefore, the kernel of $\mu_{\pi}$ is trivial.

Remark 4.10. Proposition 4.9 generalises [67, Theorem 2.4], which states that every finite covering of $\mathbb{N}$ strongly splits. 


\subsection{Covering reducts of trivial coverings}

In this subsection, we describe the automorphism groups of covering reducts of a trivial finite covering of a structure in $\mathcal{U}^{*}$. In particular, we show that there are always finitely many of them.

Remark 4.11. Let $\mathfrak{A}$ be a strongly trivial covering of a unary structure $\mathfrak{B}$ with orbits $O_{1}, \ldots, O_{k}$. Then, as in Remark 2.29, the elements of the structure $\mathfrak{A}$ can be identified with the elements of $\bigsqcup_{i=1}^{k}\left(F_{i} \times O_{i}\right)$ for some finite sets $F_{i}$ so that $\operatorname{Aut}(\mathfrak{Q})$ consists of exactly those permutations which preserve the first coordinate and stabilise the sets $O_{i}$ in the second coordinate. In this case, Aut(2I) can be written as $\prod_{i \in\{1, \ldots, k\}}\left\{\operatorname{id}_{F_{i}}\right\}$ \& $\operatorname{Sym}\left(O_{i}\right)$. For convenience, we will always assume that the sets $F_{i}$ are pairwise disjoint.

Remark 4.12. It follows from the description of strongly trivial coverings of unary structures in Remark 4.11 that every (reduct of a) strongly trivial covering structure of a structure from $\mathcal{U}$ has a first-order interpretation over $(\mathbb{N} ;=)$.

Throughout this subsection, let us fix a structure $\mathfrak{B} \in \mathcal{U}^{*}$ and a trivial finite covering map $\pi: \mathfrak{U} \rightarrow \mathfrak{B}$. Let $O_{1}, \ldots, O_{k}$ be the orbits of $\mathfrak{B}$. We identify the elements of the structure $\mathfrak{A}$ with the elements of $\bigsqcup_{i=1}^{k}\left(F_{i} \times O_{i}\right)$ for some disjoint finite sets $F_{1}, \ldots, F_{k}$ as explained in Remark 4.11, and define $F:=\bigsqcup_{i=1}^{k} F_{i}$.

Definition 4.13. We define two permutation groups.

- Let $S$ be the group of all permutations of $A$ which fix the sets $F_{i} \times O_{i}$ for $i \in\{1, \ldots, k\}$ and which preserve the congruence $\sim_{\pi}$.

- Let $N$ be the group of all permutations of $A$ which fix all fibers of $\pi$ setwise (i.e., $N$ is the kernel of the map $\mu_{\pi}: S \rightarrow \operatorname{Aut}(\mathfrak{B})$ ).

The following statements are direct consequences of the definitions above.

Proposition 4.14. A first-order reduct $\mathfrak{C}$ of $\mathfrak{A}$ is a covering reduct of $\mathfrak{B}$ with respect to the covering $\pi$ if and only if $\operatorname{Aut}(\mathfrak{C}) \subseteq S$.

The group $S$ can be written as a semidirect product $N \rtimes \operatorname{Aut}(\mathfrak{H})$.

Proof. The first statement follows easily from the definition using that

$$
\operatorname{Aut}(\mathfrak{B})=\prod_{i=1}^{k} \operatorname{Sym}\left(O_{i}\right) .
$$


Since $N$ is the kernel of the homomorphism $\mu_{\pi}: S \rightarrow \operatorname{Aut}(\mathfrak{B})$, we have $N \triangleleft S$. It is obvious that $\operatorname{Aut}(\mathfrak{U}) \leq S$ and that $S=N$ Aut( $(\mathfrak{U})$. Since $\pi$ is a trivial covering map, it follows that the kernel of $\left.\mu_{\pi}\right|_{\text {Aut( }(\mathscr{V})}$ is trivial, that is, $N \cap \operatorname{Aut}(\mathfrak{V})=\left\{\operatorname{id}_{A}\right\}$.

The following lemma is a direct consequence of item (2) of Proposition 4.14. Let $H$ and $K$ be subgroups of the same group. Then we say that $H$ normalises $K$ if $H$ is a subgroup of the normaliser of $K$, i.e., for every $h \in H$, we have that

$$
\left\{h^{-1} k h \mid k \in K\right\}=K \text {. }
$$

Lemma 4.15. The mapping $G \mapsto G \cap N$ defines a bijection between the closed subgroups of $S$ that contain Aut( $(\mathfrak{L})$ and the closed subgroups of $N$ which are normalised by Aut(2) . The inverse map is $H \mapsto H \rtimes \operatorname{Aut}(\mathfrak{U})$.

Proof. If $H$ is a subgroup of $N$ which is normalised by $\operatorname{Aut}(\mathfrak{U})$, then the group generated by $H$ and $\operatorname{Aut}(\mathfrak{U})$ can be written as a product $H$ Aut(2). Since

$$
H \cap \operatorname{Aut}(\mathfrak{U}) \subseteq N \cap \operatorname{Aut}(\mathfrak{U})=\left\{\operatorname{id}_{A}\right\},
$$

it follows that this group can be written as a semidirect product $H \rtimes \operatorname{Aut}(\mathfrak{H})$. Then $(H \rtimes \operatorname{Aut}(\mathfrak{U})) \cap N=H$.

We claim that if $H$ is closed, then so is $H \rtimes \operatorname{Aut}(\mathfrak{Q})$. Let

$$
\alpha_{1}, \alpha_{2}, \ldots \in H \rtimes \operatorname{Aut}(\mathfrak{U})
$$

be a sequence converging to some $\alpha \in \operatorname{Sym}(A)$. Let $\beta_{i}$ (and $\beta$ ) be the unique element in $\operatorname{Aut}(\mathfrak{U})$ for which $\mu_{\pi}\left(\beta_{i}\right)=\mu_{\pi}\left(\alpha_{i}\right)$ (and $\mu_{\pi}(\beta)=\mu_{\pi}(\alpha)$ ), that is, $\alpha_{i} \beta_{i}^{-1} \in H$ (and $\alpha \beta^{-1} \in H$ ). Since $\mu_{\pi}$ is continuous, it follows that $\left(\beta_{i}\right)_{i}$ converges to $\beta$. Hence, the sequence $\left(\alpha_{i} \beta_{i}^{-1}\right)_{i}$ converges to $\alpha \beta^{-1}$. Since $\alpha_{i} \beta_{i}^{-1} \in H$ and $H$ is closed, it follows that $\alpha \beta^{-1} \in H$, and hence

$$
\alpha \in H \operatorname{Aut}(\mathfrak{Q})=H \rtimes \operatorname{Aut}(\mathfrak{Q}) .
$$

Therefore, $H \rtimes \operatorname{Aut}(\mathfrak{A})$ is closed.

Let $G$ be a subgroup of $S$ containing $\operatorname{Aut}(\mathfrak{U})$. We claim that Aut( $\mathfrak{U})$ normalises $G \cap N$. Indeed, let $g \in \operatorname{Aut}(\mathfrak{U})$. Then $\left\{g^{-1} h g \mid h \in G \cap N\right\}=G \cap N$ since $N$ is a normal subgroup of $S$ which contains $\operatorname{Aut}(\mathfrak{U})$. Since

$$
(G \cap N) \cap \operatorname{Aut}(\mathfrak{U}) \subseteq N \cap \operatorname{Aut}(\mathfrak{U})=\left\{\operatorname{id}_{A}\right\},
$$

it follows that $G$ can be written as $G=(G \cap N) \rtimes \operatorname{Aut}(\mathfrak{U})$. Moreover, it is clear that if $G$ is closed, then so is $G \cap N$.

We have obtained that the mappings defined in the lemma are inverses of each other, which also implies that they both are bijections. 
Hence, in order to classify the covering reducts of $\mathfrak{A}$, it is enough to classify those closed subgroups of $N$ which are normalised by $\operatorname{Aut}(\mathfrak{U})$. If $K$ is a normal subgroup of a group $G$, then we write that two elements $x_{1}, x_{2} \in G$ are the same modulo $K$ if they represent the same element in the factor group $G / K$, i.e., if $x_{1} x_{2}^{-1} \in K$.

Definition 4.16. Let $H$ be a subgroup of

$$
\prod_{i=1}^{k} \operatorname{Sym}\left(F_{i}\right) \subseteq \operatorname{Sym}(F) .
$$

For $i \in\{1, \ldots, k\}$, let $H_{i}:=\left.H_{F \backslash F_{i}}\right|_{F_{i}} \subseteq \operatorname{Sym}\left(F_{i}\right)$, and let $N_{i}$ be a subgroup of $H_{i}$ normalised by $\left.H\right|_{F_{i}}$ for every $i \in\{1, \ldots, k\}$ so that if $\left|O_{i}\right|=1$, we have $N_{i}=H_{i}$. We write $N\left(H, N_{1}, \ldots, N_{k}\right)$ for the group of all permutations $\alpha \in N$ such that

- for every $i \in\{1, \ldots, k\}$ and for every $x_{i} \in O_{i}$, there is a permutation $\gamma \in H$ such that the action of $\alpha$ on the first coordinate of the fiber $F_{i} \times\left\{x_{i}\right\}$ is exactly the $i$-th coordinate of $\gamma$,

- for every $i \in\{1, \ldots, k\}$ and $x, y \in O_{i}$, the actions of $\alpha$ on the first coordinate of the fibers $F_{i} \times\{x\}$ and $F_{i} \times\{y\}$ are the same modulo $N_{i}$.

It follows directly from the definition that $N\left(H, N_{1}, \ldots, N_{k}\right)$ is a closed subgroup of $N$ and normalised by $\operatorname{Aut}(\mathfrak{U})$. We will show that subgroups of $N$ with these properties are of the form $N\left(H, N_{1}, \ldots, N_{k}\right)$.

Definition 4.17. Let $G$ be a subgroup of $N$ which is normalised by Aut( $(\mathscr{Y})$.

- Let $H(G)$ be the subgroup of $\prod_{i=1}^{k} \operatorname{Sym}\left(F_{i}\right)$ containing all permutations $\gamma$ such that there exists a permutation $\alpha \in G$ and for every $i \in\{1, \ldots, k\}$ an element $x_{i} \in O_{i}$ such that the action of $\alpha$ on the first coordinate of the fiber $F_{i} \times\left\{x_{i}\right\}$ is exactly the $i$-th coordinate of $\gamma$.

- $H_{i}(G):=\left.H(G)_{F \backslash F_{i}}\right|_{F_{i}}$.

- Let $N_{i}(G)$ be the group of all $\gamma \in \operatorname{Sym}\left(F_{i}\right)$ such that there exists a permutation $\alpha \in G$ and an $x \in O_{i}$ such that the action of $\alpha$ on the first coordinate of the fiber $F_{i} \times\{x\}$ equals $\gamma$ and $\alpha$ fixes every element of $A \backslash\left(F_{i} \times\{x\}\right)$.

Remark 4.18. Since $\operatorname{Aut}(\mathfrak{U})=\prod_{i=1}^{k} \operatorname{Sym}\left(O_{i}\right)$ normalises $G$, it does not matter which elements $x_{i} \in O_{i}$ we take in the definition of $H(G)$ and $N_{i}(G)$. It follows that $H(G), H_{i}(G)$, and $N_{i}(G)$ are indeed groups. 
Remark 4.19. It is clear from the definition that $N_{i}(G) \leq H_{i}(G)$, and if $\left|O_{i}\right|=1$, then $N_{i}(G)=H_{i}(G)$.

Example 4.20. A simple example for $k=1$ where $H_{1} \neq N_{1}$ is Example 2.21, where $N_{1}=\{\mathrm{id}\}$ and $H_{1}=\mathbb{Z}_{2}$.

Lemma 4.21. Let $H, N_{1}, \ldots, N_{k}$ be the groups as introduced in Definition 4.16. Then $H\left(N\left(H, N_{1}, \ldots, N_{k}\right)\right)=H$ and $N_{i}\left(N\left(H, N_{1}, \ldots, N_{k}\right)\right)=N_{i}$.

Proof. It follows directly from the definition that $H\left(N\left(H, N_{1}, \ldots, N_{k}\right)\right)=H$. For the second claim, let us assume that $i \in\{1, \ldots, k\}, x \in O_{i}$, and that $\alpha \in N$ fixes every element of $A \backslash\left(F_{i} \times\{x\}\right)$. Let $H_{i}$ be the group as introduced in Definition 4.16. We have to show that $\alpha \in N\left(H, N_{1}, \ldots, N_{k}\right)$ if and only if $\alpha$ acts on the first coordinate of the fiber $F_{i} \times\{x\}$ as a permutation in $N_{i}$.

For the "if", let us assume that the action of $\alpha$ on the first coordinate of the fiber $F_{i} \times\{x\}$, which we denote by $\gamma_{i}$, is an element of $N_{i}$. We show that

$$
\alpha \in N\left(H, N_{1}, \ldots, N_{k}\right) .
$$

First we show that $\alpha$ satisfies the first bullet of Definition 4.16. Arbitrarily choose $j \in\{1, \ldots, k\}$ and $x_{j} \in O_{j}$. Let $\gamma_{j}$ denote the action of $\alpha$ on the fiber $F_{j} \times\left\{x_{j}\right\}$, and let $\gamma=\prod_{j=1}^{k} \gamma_{j}$. We have to show that $\gamma \in H$. It follows from the definition of $\alpha$ that $\gamma_{j}=\operatorname{id}\left(F_{j}\right)$ if $j \neq i$. Therefore, it is enough to show that

$$
\gamma_{i} \in H_{i}=\left.H_{F \backslash F_{i}}\right|_{F_{i}} .
$$

If $x_{i}=x$, then $\gamma_{i} \in N_{i}$, and if $x_{i} \neq x$, then by definition, $\gamma_{i}=\operatorname{id}\left(F_{i}\right) \in N_{i}$. In either case, we have $\gamma_{i} \in N_{i} \leq H_{i}$. It remains to show that $\alpha$ satisfies the second bullet of Definition 4.16. In fact, we show something stronger. We show that, for every $j \in\{1, \ldots, k\}$ and $y \in O_{j}$, the action of $\alpha$ on $F_{j} \times\{y\}$ is an element of $N_{j}$. If $y=x$, and thus $j=i$, then this is exactly our assumption on $\alpha$. If $y \neq x$, then this action is trivial since, by definition, $\alpha$ fixes every element in $A \backslash\left(F_{i} \times\{x\}\right)$.

For the other direction, let us assume that $\alpha \in N\left(H, N_{1}, \ldots, N_{k}\right)$. We distinguish two cases. If $\left|O_{i}\right|=1$, then by definition, $\alpha$ acts on the first coordinate of the fiber $F_{i} \times\{x\}$ as a permutation in $H_{i}\left(N\left(H, N_{1}, \ldots, N_{k}\right)\right)$, and by Remark 4.19, we have

$$
\begin{aligned}
N_{i}\left(N\left(H, N_{1}, \ldots, N_{k}\right)\right) & =H_{i}\left(N\left(H, N_{1}, \ldots, N_{k}\right)\right) \\
& =\left.\left(H\left(N\left(H, N_{1}, \ldots, N_{k}\right)\right)_{F \backslash F_{i}}\right)\right|_{F_{i}} \\
& =\left.H_{F \backslash F_{i}}\right|_{F_{i}}=H_{i}=N_{i},
\end{aligned}
$$


where the last equality follows from the assumptions on the groups $H_{i}$ and $N_{i}$ in Definition 4.16.

If $\left|O_{i}\right|>1$, then let $y$ be an element in $O_{i}$ different from $x$. Then if

$$
\alpha \in N\left(H, N_{1}, \ldots, N_{k}\right),
$$

then on one hand, we know that the action of $\alpha$ on the first coordinate of the fibers $F_{i} \times\{x\}$ and $F_{i} \times\{y\}$ are the same modulo $N_{i}$. On the other hand, we know by the definition of $\alpha$ that its action on the fiber $F_{i} \times\{y\}$ is trivial. Therefore, the action of $\alpha$ on the first coordinate of the fiber $F_{i} \times\{x\}$ is an element of $N_{i}$.

Definition 4.22. Let $N_{i}$ be a subgroup of $\operatorname{Sym}\left(F_{i}\right)$ for some $i \in\{1, \ldots, k\}$. Then $N^{*}\left(N_{1}, \ldots, N_{k}\right)$ is defined to be the closure of the group generated by all permutations $\alpha$ for which there exists an $i$ and $x \in O_{i}$ such that the action of $\alpha$ on the first coordinate of the fiber $F_{i} \times\{x\}$ is in $N_{i}$ and $\alpha$ fixes every element of $A \backslash\left(F_{i} \times\{x\}\right)$.

It follows easily from the definition that $N^{*}\left(N_{1}, \ldots, N_{k}\right)$ is contained in every closed group $G \leq N$ normalised by $\operatorname{Aut}(\mathfrak{Q})$ with $N_{i}(G)=N_{i}$. It is also easy to see that, in fact, $N^{*}\left(N_{1}, \ldots, N_{k}\right)=N\left(\prod_{i=1}^{k} N_{i}, N_{1}, \ldots, N_{k}\right)$ (using the notation from Definition 4.16).

Example 4.23. Let $\mathfrak{B}:=\mathbb{N}$ and $\mathfrak{A}$ a strongly trivial finite covering structure of $\mathfrak{B}$ with fibers of size four. Then the covering structure from Example 2.22 is a covering reduct $\mathfrak{C}$ of $\mathfrak{A}$. Let $G:=\operatorname{Aut}(\mathfrak{C})$. As $G$ is transitive, we have $S=N$ and $k=1$ in Definition 4.17. Then $H(G)=\prod_{i=1}^{k} \mathbb{Z}_{4}$ and $N_{1}(G)=\mathbb{Z}_{2}$.

Lemma 4.24. Let $G \leq N$ be normalised by Aut( $(\mathfrak{)})$. Then $\left.N_{i}(G) \triangleleft H(G)\right|_{F_{i}}$.

Proof. Let $\left.\alpha \in H(G)\right|_{F_{i}}$ and $\beta \in N_{i}(G)$. Let $\gamma \in G$ be an element witnessing $\left.\alpha \in H(G)\right|_{F_{i}}$. Let $x \in O_{i}$, and let $\delta \in G$ be an element witnessing $\delta \in N_{i}(G)$ on the fiber $F_{i} \times\{x\}$. Then the element $\gamma^{-1} \delta \gamma \in G$ witnesses the fact that

$$
\alpha^{-1} \beta \alpha \in N_{i}(G) \text {. }
$$

Lemma 4.25. Let $G$ be a closed subgroup of $N$ normalised by Aut( $(2)$. Let $\alpha \in G$ and $u, v \in O_{i}$. Then the actions of $\alpha$ on the first coordinate of the fibers $F_{i} \times\{u\}$ and $F_{i} \times\{v\}$ are the same modulo $N_{i}(G)$.

Proof. Let $\alpha_{u}$ and $\alpha_{v}$ denote the action of $\alpha$ on the first coordinate of the fibers of $u$ and $v$, respectively, so $\alpha_{u}, \alpha_{v} \in \operatorname{Sym}\left(F_{i}\right)$. For $\beta \in \operatorname{Aut}(\mathfrak{B})$, we write $\pi^{-1}(\beta)$ for the unique $\gamma \in \operatorname{Aut}(\mathfrak{H})$ such that $\mu_{\pi}(\gamma)=\beta$. Let $\beta=(u v) \in \operatorname{Aut}(\mathfrak{B})$. Let

$$
\gamma:=\alpha^{-1}\left(\pi^{-1}(\beta)\right)^{-1} \alpha \pi^{-1}(\beta) .
$$


Then the action of $\gamma$ on the first coordinate of the fiber $F_{i} \times\{u\}$ is $\alpha_{u}^{-1} \alpha_{v}$. On the other hand, $\gamma$ fixes every element of $A \backslash F_{i} \times\{u, v\}$. Now let $v_{1}, v_{2}, \ldots$ be pairwise distinct elements of $O_{i}$. Let $\beta_{i}:=\left(u v_{i}\right)$ and $\gamma_{i}:=\left(\pi^{-1}\left(\beta_{i}\right)\right)^{-1} \gamma \pi^{-1}\left(\beta_{i}\right)$. Then $\gamma_{i}$ acts on the first coordinate of the fiber $F_{i} \times\{u\}$ as $\alpha_{u}^{-1} \alpha_{v}$, and it fixes every element outside $F_{i} \times\left\{u, v_{i}\right\}$. Therefore, the permutations $\gamma_{i}$ converge to a permutation $\gamma^{\prime}$ which acts on the first coordinate of the fiber $F_{i} \times\{u\}$ as $\alpha_{u}^{-1} \alpha_{v}$, and fixes every element outside $F_{i} \times\{u\}$. By our assumption, $G$ is closed, so $\gamma^{\prime} \in G$. By definition, this implies that $\alpha_{u}^{-1} \alpha_{v} \in N_{i}(G)$.

Proposition 4.26. Let $\mathfrak{C}$ be a covering reduct of $\mathfrak{A}$ and $G:=\operatorname{Aut}(\mathfrak{C}) \cap N$. Then

$$
G=N\left(H(G), N_{1}(G), \ldots, N_{k}(G)\right) .
$$

Proof. By Remark 4.19, we know that $N_{i}(G) \leq H_{i}(G)=\left.H(G)_{F \backslash F_{i}}\right|_{F_{i}}$, and if $\left|O_{i}\right|=1$, then $N_{i}(G)=H_{i}(G)$. Lemma 4.24 implies that $\left.H(G)\right|_{F_{i}}$ normalises $N_{i}(G)$. Therefore, the group $G=N\left(H(G), N_{1}(G), \ldots, N_{k}(G)\right)$ is well-defined.

We first show that $G \leq N\left(H(G), N_{1}(G), \ldots, N_{k}(G)\right)$. Let $\alpha \in G$. Then the definition of the group $H(G)$ implies that $\alpha$ satisfies the first item in the definition of $N\left(H(G), N_{1}(G), \ldots, N_{k}(G)\right)$. By Lemma 4.25, $\alpha$ also satisfies the second item of this definition, and hence $\alpha \in N\left(H(G), N_{1}(G), \ldots, N_{k}(G)\right)$.

Now let $\alpha \in N\left(H(G), N_{1}(G), \ldots, N_{k}(G)\right)$ be arbitrary. Let $u_{i} \in O_{i}$ be arbitrary elements. By Lemma 4.21, we have

$$
H\left(N\left(H(G), N_{1}(G), \ldots, N_{k}(G)\right)\right)=H(G) .
$$

This implies that there exists an $\alpha^{\prime} \in G$ such that, for every $i \in\{1, \ldots, k\}$, the actions of $\alpha$ and $\alpha^{\prime}$ agree on $F_{i} \times\left\{u_{i}\right\}$. For $v \in O_{i}$, let $\alpha_{v}$ and $\alpha_{v}^{\prime}$ denote the action of $\alpha$ and $\alpha^{\prime}$, respectively, on the fiber $F_{i} \times\{v\}$. We claim that, for all $v \in O_{i}$, it holds that $\alpha_{v}\left(\alpha_{v}^{\prime}\right)^{-1} \in N_{i}$. By Lemma 4.21, we have

$$
N_{i}\left(N\left(H(G), N_{1}(G), \ldots, N_{k}(G)\right)\right)=N_{i}(G),
$$

and hence, by Lemma 4.25, it follows that $\alpha_{v} \alpha_{u_{i}}^{-1} \in N_{i}(G)$, and

$$
\alpha_{v}^{\prime}\left(\alpha_{u_{i}}^{\prime}\right)^{-1}=\alpha_{v}^{\prime} \alpha_{u_{i}}^{-1} \in N_{i}(G),
$$

and hence

$$
\alpha_{v}\left(\alpha_{v}^{\prime}\right)^{-1}=\alpha_{v} \alpha_{u_{i}}^{-1} \alpha_{u_{i}}\left(\alpha_{v}^{\prime}\right)^{-1}=\alpha_{v} \alpha_{u_{i}}^{-1}\left(\alpha_{v}^{\prime} \alpha_{u_{i}}^{-1}\right)^{-1} \in N_{i}(G) .
$$

This implies that $\alpha\left(\alpha^{\prime}\right)^{-1} \in N\left(\prod_{i=1}^{k} N_{i}(G), N_{1}(G), \ldots, N_{k}(G)\right)=N^{*}\left(N_{1}(G), \ldots, N_{k}(G)\right) \subseteq G$. Therefore, $\alpha=\left(\alpha\left(\alpha^{\prime}\right)^{-1}\right) \alpha^{\prime} \in G$. 
Remark 4.27. Proposition 4.26 generalises [67, Theorem 3.1] from $\operatorname{Sym}(\mathbb{N})$ to arbitrary automorphism groups of structures in $\mathcal{U}^{*}$.

Corollary 4.28. $\mathfrak{A}$ has finitely many covering reducts with respect to $\pi$.

Proof. By Lemma 4.15 and item (1) of Proposition 4.14, it is enough to show that $N$ has finitely many closed subgroups which are normalised by Aut(2). By Proposition 4.26 and Remark 4.19, these groups can be characterised by a subgroup $H$ of $\prod_{i=1}^{k} \operatorname{Sym}\left(F_{i}\right)$ and a system of subgroups $N_{i} \leq H_{i}$. Then the statement of the corollary follows from the fact that there are finitely many choices for these groups.

\section{Finite coverings of reducts of unary structures}

In this section, we show that every structure in $F(R(U))$ is a quasi-covering reduct (introduced in Definition 5.9) of a strongly trivial covering of some structure in $U^{*}$ (Proposition 5.11), and that there are only finitely many of such reducts for each structure in $R(\mathcal{U})$ (Theorem 5.10). Moreover, we observe (Remark 5.8) that $F(R(\mathcal{U})) \subseteq R(F(\mathcal{U}))$.

\subsection{The Ramsey property and canonical functions}

Let $\mathfrak{A}, \mathfrak{B}$ be structures. A function $f: A \rightarrow B$ is called canonical from $\mathfrak{A}$ to $\mathfrak{B}$ if, for every $t \in A^{n}$ and $\alpha \in \operatorname{Aut}(\mathfrak{Q})$, there exists $\beta \in \operatorname{Aut}(\mathfrak{B})$ such that

$$
f(\alpha(t))=\beta(f(t)) .
$$

Hence, a canonical function $f$ induces, for every $n$, a function from the orbits of $n$-tuples of $\operatorname{Aut}(\mathfrak{Q})$ to the orbits of $n$-tuples of $\operatorname{Aut}(\mathfrak{B})$; these functions will be called the behaviour of $f$. Canonical functions as a tool to classify reducts of homogeneous structures with finite relational signature have been introduced in [17] and used in $[1,2,11,13,20,59]$. The existence of certain canonical functions in the automorphism group of a structure $\mathfrak{A}$ is typically shown using Ramsey properties of $\mathfrak{A}$. We will not introduce Ramsey structures here; all that is needed is the wellknown fact that $(\mathbb{Q} ;<)$ is Ramsey, and the following result from [17]. A structure is called ordered if the signature contains a binary relation symbol that denotes a (total) linear ordering of the domain.

Lemma 5.1 (see [19]). Let $\mathfrak{D}$ be an ordered homogeneous Ramsey structure with finite relational signature, and let $f: D \rightarrow D$ be a function. Then there exists a function $g \in \overline{\{\alpha \circ f \circ \beta \mid \alpha, \beta \in \operatorname{Aut}(\mathfrak{D})\}}$ which is canonical as a function from $\mathfrak{D}$ to $\mathfrak{D}$. 
The following is an easy consequence of the definitions.

Lemma 5.2. Let $\mathfrak{A}$ be a homogeneous structure with finite relational signature, and let $\mathfrak{B}$ be a first-order reduct of $\mathfrak{X}$. If $f$ and $g$ are canonical functions from $\mathfrak{A}$ to $\mathfrak{B}$ with the same behaviour, then $\overline{\operatorname{Aut}(\mathfrak{B}) \cup\{f\}}=\overline{\operatorname{Aut}(\mathfrak{B}) \cup\{g\}}$.

The next lemma follows from the observation that if $\mathfrak{U}$ is homogeneous with a relational signature of maximal arity $k$, then the behaviour of a canonical function $f$ from $\mathfrak{A}$ to $\mathfrak{B}$ is fully determined by the function induced by $f$ on the orbits of $k$-tuples (see [22, in particular the comments at the end of Section 4.1]).

Lemma 5.3. Let $\mathfrak{A}$ be a homogeneous structure with finite relational signature, and let $\mathfrak{B}$ be $\omega$-categorical. Then there are finitely many behaviours of canonical functions from $\mathfrak{A}$ to $\mathfrak{B}$.

The structures in $F\left(U^{*}\right)$ have homogeneous expansions with finite relational signature which we describe next.

Lemma 5.4. Let $\mathfrak{B} \in \mathcal{U}^{*}$, and let $\pi: \mathfrak{U} \rightarrow \mathfrak{B}$ be a strongly trivial finite cover. Then

(1) $\mathfrak{U}$ is interdefinable with a homogeneous structure $\mathfrak{C}$ with finite relational signature,

(2) $\mathfrak{A}$ is a first-order reduct of an ordered homogeneous Ramsey structure $\mathfrak{D}$ with finite relational signature.

Proof. Let $O_{1}, \ldots, O_{k}$ be the orbits of $\mathfrak{B}$. Following Remark 4.11, we can assume that $\mathfrak{U}=\bigsqcup_{i=1}^{k}\left(F_{i} \times O_{i}\right)$ for some finite sets $F_{i}$, and that Aut(2) consists of all permutations which preserve the first coordinate and stabilise the sets $O_{i}$ on the second coordinate. For each $i \leq k$ and $s \in F_{i}$, we define the unary relation $U_{i, s}:=\left\{(s, u) \mid u \in O_{i}\right\}$. Let $\mathbb{E}$ be the relational structure with domain $A$ and the relations $U_{i, s}$ and $\sim_{\pi}$. Then $\operatorname{Aut}(\mathfrak{C})=\operatorname{Aut}(\mathfrak{U})$. Hence, $\mathfrak{T}$ and $\mathfrak{C}$ are interdefinable. It is easy to see that $\mathbb{C}$ is homogeneous. This proves (1).

To prove item (2), we define an ordering $<$ on $\mathfrak{B}$ as follows. For each infinite orbit $O_{i}$, let us fix an ordering $<_{i}$ on $O_{i}$ which is isomorphic to $(\mathbb{Q} ;<)$. Let us also fix an ordering of $\prec_{i}$ on $F_{i}$ for all $i$. Then $<$ is defined as follows.

- If $\pi(x) \in O_{i}, \pi(y) \in O_{j}$ and $i<j$, then $x<y$,

- If $\pi(x), \pi(y) \in O_{i}$ and $\pi(x)<_{i} \pi(y)$, then $x<y$,

- If $\pi(x)=\pi(y) \in O_{i}$ and $x^{\prime}$ and $y^{\prime}$ are the projections of $x$ and $y$ to the first component, then $x<y$ if and only if $x^{\prime} \prec_{i} y^{\prime}$. 
To show that the expansion $\mathfrak{D}$ of $\mathfrak{C}$ by the ordering $<$ has the Ramsey property, we use the fact that if a structure is the disjoint union of substructures induced by definable subsets, and the substructures are Ramsey, then the structure itself is Ramsey (see [10]). For each $i \leq k$, let $\mathfrak{D}_{i}$ be the substructure of $\mathfrak{D}$ induced by $\pi^{-1}\left(O_{i}\right)$. Note that $\pi^{-1}\left(O_{i}\right)=\bigcup_{s \in F_{i}} U_{i, s}$ and hence is definable in $\mathfrak{D}$. If $O_{i}$ is infinite, then $\operatorname{Aut}\left(\mathfrak{D}_{i}\right)$ is topologically isomorphic to $\operatorname{Aut}(\mathbb{Q} ;<)$. The property of a structure of being Ramsey is a property of the automorphism group of the structure, viewed as a topological group (again, see [10]). It follows that $\mathfrak{D}$ is Ramsey.

\subsection{Reducts of finite covers of reducts of unary structures}

Let $\mathfrak{B} \in R(\mathcal{U})$, and let $\pi: \mathfrak{Q} \rightarrow \mathfrak{B}$ be a finite covering map. In this section, we study the closed supergroups $G$ of $\operatorname{Aut}(\mathfrak{U})$ that preserve $\sim_{\pi}$ such that $\mu_{\pi}(G)$ also preserves the congruence $\nabla(\mathfrak{B})$.

Lemma 5.5. Let $\mathfrak{B} \in \mathcal{U}^{*}$, and let $\pi: \mathfrak{A} \rightarrow \mathfrak{B}$ be a strongly trivial finite covering map. Let $O_{1}, \ldots, O_{k}$ be the orbits of $\mathfrak{B}$, and let $\mathfrak{D}$ be the ordered homogeneous finite signature Ramsey expansion of $\mathfrak{U}$ from Lemma 5.4. Suppose that $f \in \operatorname{Sym}(A)$ preserves $\sim_{\pi}$ and that $\mu_{\pi}(f)$ preserves the partition $P:=\left\{O_{1}, \ldots, O_{k}\right\}$. Then the monoid $M:=\overline{\langle\operatorname{Aut}(\mathfrak{U}), f\rangle}$ contains a surjective map $h$ which is canonical from $\mathfrak{D}$ to $\mathfrak{A}$ and such that $\mu_{\pi}(h)$ has the same action on $P$ as $\mu_{\pi}(f)$.

Proof. By Lemma 5.1, we obtain that there exists a function

$$
g \in \overline{\{\alpha f \beta \mid \alpha, \beta \in \operatorname{Aut}(\mathfrak{D})\}} \subseteq M
$$

which is canonical from $\mathfrak{D}$ to $\mathfrak{D}$. Since $g \in M$, it follows that $g$ preserves $\sim_{\pi}$. But note that $g$ is not necessarily surjective. For $m \in M$, define $\mu_{\pi}(m)$ by

$$
x \mapsto \pi\left(m\left(\pi^{-1}(x)\right)\right)
$$

as in the case of bijective functions. Since every automorphism of $\mathfrak{D}$ preserves the orbits $O_{1}, \ldots, O_{k}$, it follows that if $\mu_{\pi}(f)\left(O_{i}\right)=O_{j}$, then $\mu_{\pi}(g)\left(O_{i}\right) \subseteq O_{j}$.

If $\mu_{\pi}(f)\left(O_{i}\right)=O_{j}$, then $\left|F_{O_{i}}\right|=\left|F_{O_{j}}\right|$ since $f$ preserves $\sim_{\pi}$ and is surjective. Therefore, for every $g^{\prime} \in M$ and $u \in B$, the restriction of $g^{\prime}$ to $U:=\pi^{-1}(u)$ is a bijection between $U$ and $\pi^{-1}\left(\mu_{\pi}\left(g^{\prime}\right)(u)\right)$. In particular, this holds for $g \in M$. If $O_{i}=\left\{u_{i}\right\}$, then $g$ defines a bijection between $\pi^{-1}\left(u_{i}\right)$ and $\pi^{-1}\left(\mu_{\pi}(f)\left(u_{i}\right)\right)$. If $O_{i}$ is infinite, then $\pi^{-1}\left(\mu_{\pi}(g)\left(O_{i}\right)\right)$ is a union of infinitely many classes of $\sim_{\pi}$. Moreover, $O_{i}$ is infinite if and only if $\mu_{\pi}(g)\left(O_{i}\right)$ is infinite. Let $e: A \rightarrow A$ be defined as $(s, u) \mapsto\left(s, \mu_{\pi}(g)(u)\right)$. Let $\mathbb{C}$ be the homogeneous structure from Lemma 5.4 which has the property that $\operatorname{Aut}(\mathfrak{C})=\operatorname{Aut}(\mathfrak{U})$. Then $e$ is an isomorphism 
between $\mathfrak{C}$ and the substructure of $\mathfrak{C}$ induced by $g(C)$. Since $\mathfrak{C}$ is homogeneous, it follows that $e \in \overline{\operatorname{Aut}(\mathfrak{C})}=\overline{\operatorname{Aut}(\mathfrak{N})}$, and so there is a sequence $e_{1}, e_{2}, \ldots \in \operatorname{Aut}(\mathfrak{U})$ which converges to $e$. Then $h_{i}:=e_{i}^{-1} g \in M$ converges to $h:=e^{-1} \circ g$, and thus $h \in M$. We claim that the mapping $h$ satisfies the conditions of the lemma. By definition, $h(A)=e^{-1}(g(A))=A$, that is, $h$ is surjective. Since $e^{-1}$ preserves the relations of $\mathfrak{C}$, it follows that the mapping $h$ is canonical from $\mathfrak{D}$ to $\mathfrak{C}$ (and therefore also from $\mathfrak{D}$ to $\mathfrak{A})$. For the same reason, $\mu_{\pi}\left(e^{-1}\right)$ preserves all orbits $O_{i}$. This implies that $\mu_{\pi}(h)$ and $\mu_{\pi}(g)$ and therefore also $\mu_{\pi}(f)$ have the same action on $\left\{O_{1}, \ldots, O_{k}\right\}$.

Lemma 5.6. Let $\mathfrak{B} \in \mathcal{U}^{*}$, and let $\pi: \mathfrak{U} \rightarrow \mathfrak{B}$ be a finite covering map. Moreover, let $O_{1}, \ldots, O_{k}$ be the orbits of $\mathfrak{B}$. Then $\operatorname{Sym}(A)$ has finitely many closed subgroups $G$ such that

- $\operatorname{Aut}(\mathfrak{N}) \subseteq G$,

- $G$ preserves $\sim_{\pi}$,

- $\mu_{\pi}(G)$ preserves the partition $\left\{O_{1}, \ldots, O_{k}\right\}$ of $B$.

Proof. By Proposition 4.9, we know that $\mathfrak{A}$ is a covering reduct of some strongly trivial covering $\mathfrak{C}$ of $\mathfrak{B}$ (with respect to $\pi$ ). Then let $\mathfrak{D}$ be the ordered homogeneous finite-signature Ramsey expansion of $\mathfrak{C}$ from Lemma 5.4. Let $G$ be a closed subgroup of $\operatorname{Sym}(A)$ as in the formulation of the lemma. Then $G$ acts on the set $\left\{O_{1}, \ldots, O_{k}\right\}$. Let $K$ be the kernel of this action. Then $K$ is closed, and the index of $K$ in $G$ is finite. Also, $K \subseteq S$, where $S$ is the group as in Definition 4.13. Therefore, $K$ is the automorphism group of a covering reduct of $\mathfrak{U}$ (Proposition 4.14). Then, by Theorem 4.2, there are finitely many possible choices for the group $K$. By Lemma 5.5, for each $f \in G$, there exists a surjective map $h \in \overline{\langle K, f\rangle}$ which is canonical from $\mathfrak{D}$ to $\mathfrak{A}$ such that $f$ and $h$ induce the same permutation $\sigma$ of $\left\{O_{1}, \ldots, O_{k}\right\}$. We claim that $K \cup\{f\}$ and $K \cup\{h\}$ generate the same group. The image of the action of $K \cup\{f\}$ and $K \cup\{h\}$ on $\left\{O_{1}, \ldots, O_{k}\right\}$ is $\langle\sigma\rangle$, and the kernel of these actions is again $K$. Therefore,

$$
[\langle K \cup\{f\}\rangle: K]=[\langle K \cup\{h\}\rangle: K]=l,
$$

where $l$ is the order of the permutation $\sigma$. In particular, the groups $\langle K \cup\{f\}\rangle$ and $\langle K \cup\{h\}\rangle$ are closed. Hence, $h \in K \cup\{f\}$, and thus $\langle K \cup\{f\}\rangle \leq\langle K \cup\{h\}\rangle$. Then, by using equality (5.1) again, it follows that $\langle K \cup\{f\}\rangle=\langle K \cup\{h\}\rangle$.

Since $[G: K]$ is finite, each group $G$ is generated by finitely many (at most $k$ !) elements over $K$. By the previous paragraph, we can assume that each of these generators are canonical from $\mathfrak{D}$ to $\mathfrak{A}$. There are finitely many possible behaviours 
of canonical functions from $\mathfrak{D}$ to $\mathfrak{A}$ (Lemma 5.3). If two functions have the same behaviour, they generate the same group over $\operatorname{Aut}(\mathfrak{U})$ (Lemma 5.2). This implies that there are finitely many choices for the group $G$.

Theorem 5.7. Let $\mathfrak{B} \in R(\mathcal{U})$, and let $\pi: \mathfrak{U} \rightarrow \mathfrak{B}$ be a finite covering map. Then Aut( $(\mathfrak{U})$ has finitely many closed supergroups $G$ such that $G$ preserves $\sim_{\pi}$ and $\mu_{\pi}(G)$ preserves $\nabla(\mathfrak{B})$.

Proof. Let $O_{1}, \ldots, O_{k}$ be the classes of $\nabla(\mathfrak{B})$. Then, by Lemma 3.3, it follows that $\prod_{i=1}^{k} \operatorname{Sym}\left(O_{i}\right) \subseteq \operatorname{Aut}(\mathfrak{B})$. Let $\mathfrak{B}^{\prime}$ be a structure with

$$
\operatorname{Aut}\left(\mathfrak{B}^{\prime}\right)=\prod_{i=1}^{k} \operatorname{Sym}\left(O_{i}\right) .
$$

Then $\mathfrak{B}^{\prime} \in \mathcal{U}^{*}$ by Lemma 2.14 and $\nabla(\mathfrak{B})=\nabla\left(\mathfrak{B}^{\prime}\right)$. The group Aut $(\mathfrak{H})$ acts naturally on the set $\left\{O_{1}, \ldots, O_{k}\right\}$. Let $K$ be the kernel of this action, and let $\mathfrak{X}^{\prime}$ be a structure so that $\operatorname{Aut}\left(\mathfrak{U}^{\prime}\right)=K$. The action of $\operatorname{Aut}\left(\mathfrak{U}^{\prime}\right)$ on $B$ equals $\operatorname{Aut}\left(\mathfrak{B}^{\prime}\right)$. Therefore, $\pi: \mathfrak{X}^{\prime} \rightarrow \mathfrak{B}^{\prime}$ is a finite cover. Then the statement of the theorem follows from Lemma 5.6 and from the fact that the orbits of $\mathfrak{B}^{\prime}$ are exactly the classes of the congruence $\nabla(\mathfrak{B})$.

Proposition 5.8. $F(R(U)) \subseteq R^{<\infty}\left(F\left(U^{*}\right)\right)$.

Proof. Let $\mathfrak{U} \in F(R(\mathcal{U})$ ). Following the notation of the proof of Theorem 5.7, we have that $\left[\operatorname{Aut}(\mathfrak{U}): \operatorname{Aut}\left(\mathfrak{U}^{\prime}\right)\right]=[\operatorname{Aut}(\mathfrak{U}): K]$ is finite since $K$ is defined as the kernel of the action of $\operatorname{Aut}(\mathfrak{U})$ on the set $\left\{O_{1}, \ldots, O_{k}\right\}$. As we saw in the proof of Theorem 5.7, we have $\mathfrak{U}^{\prime} \in F\left(\mathcal{U}^{*}\right)$. Hence, $\mathfrak{A} \in R^{<\infty}\left(F\left(\mathcal{U}^{*}\right)\right)$.

Later, we will see (in Theorem 6.29) that, in fact, $F(R(U))=R^{<\infty}\left(F\left(U^{*}\right)\right)$. The following definition of quasi-covering reducts is needed for a model-theoretic reformulation of Theorem 5.7, which is given in Theorem 5.10 below.

Definition 5.9. Let $\mathfrak{B}$ be $\omega$-categorical, and let $\pi: \mathfrak{U} \rightarrow \mathfrak{B}$ be a finite cover. A firstorder reduct $\mathfrak{E}$ of $\mathfrak{A}$ is called a quasi-covering reduct of $\mathfrak{A}$ with respect to $\pi$ if $\operatorname{Aut}(\mathfrak{C})$ preserves $\sim_{\pi}$ and $\mu_{\pi}(\operatorname{Aut}(\mathfrak{C})) \subseteq \operatorname{Sym}(B)$ preserves $\nabla(\mathfrak{B})$.

Theorem 5.10. Let $\mathfrak{B} \in R(\mathcal{U})$, and let $\pi: \mathfrak{A} \rightarrow \mathfrak{B}$ be a finite cover. Then $\mathfrak{A}$ has finitely many quasi-covering reducts with respect to $\pi$.

Proof. The statement follows immediately from Theorem 5.7 and Definition 5.9. 
Proposition 5.11. Let $\mathfrak{B} \in R(\mathcal{U})$, and let $\pi: \mathfrak{A} \rightarrow \mathfrak{B}$ be a finite cover. Then $\mathfrak{A}$ is a quasi-covering reduct of a strongly trivial covering of some structure in $U^{*}$.

Proof. Let us define the structures $\mathfrak{U}^{\prime}$ and $\mathfrak{B}^{\prime}$ as in the proof of Theorem 5.7. Then $\mathfrak{U}$ is a finite quasi-covering reduct of $\mathfrak{U}^{\prime}$ with respect to the covering map $\pi$. The map $\pi: \mathfrak{U}^{\prime} \rightarrow \mathfrak{B}^{\prime}$ is a finite covering, and $\mathfrak{B}^{\prime} \in \mathcal{U}^{*}$. By Proposition 4.9, $\mathfrak{U}^{\prime}$ is a covering reduct of some strongly trivial covering of $\mathfrak{B}^{\prime}$. Therefore, $\mathfrak{A}$ is a quasicovering reduct of a strongly trivial covering of $\mathfrak{B}^{\prime} \in \mathcal{U}^{*}$.

\section{Structures with small orbit growth}

In this section, we show that $\mathcal{K}_{\exp +}=(F \circ R)(\mathcal{U})$. We start in Section 6.1 with some observations from enumerative combinatorics that we need to obtain information about $\nabla(\mathfrak{U})$ if $\mathfrak{U} \in \mathcal{K}_{\exp +}$. In Section 6.2, we discuss the effect of stabilising a group from $\mathcal{E}_{\text {exp }+}$ at finitely many constants. Section 6.3 treats the case that $\mathfrak{A}$ is primitive; here we rely on work of Macpherson [55]. We then focus on permutation groups $G$ in $\mathscr{E}_{\exp +}$ where the congruence $\Delta(G)$ is trivial, i.e., $\Delta(G)$ is the identity congruence (Section 6.4); the general case is treated in Section 6.5. In Section 6.6, we use these results to prove Thomas' conjecture for all structures in the class $\mathcal{K}_{\exp +\text {. }}$

\subsection{Growth rates for partitions}

For $n, k \in \mathbb{N}$, let $p_{k}(n)$ be the number of partitions of the set $\{1, \ldots, n\}$ with parts of size at most $k$; this is the Sloane integer sequence A229223. Asymptotic formulas for $p_{k}(n)$ are known for $k \in\{1, \ldots, 4\}$ (called allied Bell numbers in a letter of John Riordan). We need an upper and a lower bound for all $k \in \mathbb{N}$.

Lemma 6.1. Let $\varepsilon>0$. Then $p_{k}(n) \geq n^{\left(\frac{k-1}{k}-\varepsilon\right) n}$ if $n$ is large enough.

Proof. Let $s_{k}(n)$ be the number of partitions of $\{1, \ldots, k n\}$ where all the parts contain exactly $k$ elements. Clearly, $s_{k}(1)=1$ for all $k \in \mathbb{N}$. To form a partition of $\{1, \ldots, k n\}$ for $n>1$, we first choose the class containing the number $k n$, and then we choose a partition of the remaining elements. Hence, $s_{k}$ satisfies the recursion

$$
s_{k}(n)=\left(\begin{array}{c}
k n-1 \\
k-1
\end{array}\right) s_{k}(n-1) .
$$

Since $\left(\begin{array}{c}k n-1 \\ k-1\end{array}\right) \geq n^{k-1}$, we obtain by induction that

$$
s_{k}(n) \geq n^{k-1}(n-1)^{k-1} \cdots 2^{k-1}=(n !)^{k-1} .
$$


Stirling's formula $\left(n ! \sim \sqrt{2 \pi n}\left(\frac{n}{e}\right)^{n}\right.$ for $n$ tending to infinity) implies that

$$
(n !)^{k-1} \geq n^{(k-1)\left(1-\varepsilon^{\prime}\right) n}
$$

for any $\varepsilon^{\prime}>0$ if $n$ is large enough. Hence,

$$
\begin{aligned}
p_{k}(n) & \geq s_{k}\left(\left\lfloor\frac{n}{k}\right\rfloor\right) \geq\left\lfloor\frac{n}{k}\right\rfloor^{(k-1)\left(1-\varepsilon^{\prime}\right)\left\lfloor\frac{n}{k}\right\rfloor} \\
& \geq\left(\frac{n}{k}-1\right)^{(k-1)\left(1-\varepsilon^{\prime}\right)\left(\frac{n}{k}-1\right)} \geq n^{(k-1)\left(1-\varepsilon^{\prime}\right)\left(1-\varepsilon^{\prime \prime}\right) \frac{1}{k} n} \geq n^{\left(\frac{k-1}{k}-\varepsilon\right) n}
\end{aligned}
$$

for an appropriate choices of $\varepsilon^{\prime}, \varepsilon^{\prime \prime}>0$ if $n$ is large enough.

Lemma 6.2. Let $n, k \in \mathbb{N}$. If $d>\frac{k-1}{k}$, then $p_{k}(n)<c n^{d n}$ for some $c$.

Proof. To form a partition of $\{1, \ldots, n\}$ for $n>1$, we first choose the class containing the number $n$, and then we choose a partition of the remaining elements. We thus have the following recursion formula:

$$
p_{k}(n)=\sum_{i=0}^{k-1}\left(\begin{array}{c}
n-1 \\
i
\end{array}\right) p_{k}(n-1-i) .
$$

We claim that the following inequality holds if $n$ is large enough:

$$
\sum_{i=0}^{k-1}\left(\begin{array}{c}
n-1 \\
i
\end{array}\right)(n-1-i)^{d(n-1-i)}<n^{d n} .
$$

In order to prove this, it is enough to show that if $i \leq k-1$ and $n$ is large enough, then

$$
\left(\begin{array}{c}
n-1 \\
i
\end{array}\right)(n-1-i)^{d(n-1-i)}<\frac{1}{k} n^{d n},
$$

that is,

$$
\left(\begin{array}{c}
n-1 \\
i
\end{array}\right)<\frac{1}{k}\left(\frac{n^{n}}{(n-1-i)^{n-1-i}}\right)^{d} .
$$

We have

$$
\begin{aligned}
\frac{n^{n}}{(n-1-i)^{n-1-i}} & =\prod_{j=0}^{i} \frac{(n-j)^{n-j}}{(n-j-1)^{n-j-1}} \\
& =\prod_{j=0}^{i}\left((n-j)\left(\frac{n-j}{n-j-1}\right)^{n-j-1}\right) \\
& \geq \prod_{j=0}^{i}(n-j)=n(n-1) \cdots(n-i) .
\end{aligned}
$$


This implies that, in order to show inequality (6.3), it is enough to show that

$$
\left(\begin{array}{c}
n-1 \\
i
\end{array}\right)<\frac{1}{k}(n(n-1) \cdots(n-i))^{d}
$$

if $n$ is large enough. By rearranging the inequality above, we obtain that it is equivalent to the following inequality:

$$
\frac{1}{i !}((n-1) \cdots(n-i))^{1-d}<\frac{1}{k} n^{d} .
$$

The LHS of inequality (6.4) is asymptotically $\frac{1}{i !} n^{i(1-d)}$. By our assumption, we have $d>\frac{k-1}{k}$; thus $i(1-d)<\frac{i}{k} \leq \frac{k-1}{k}<d$. This implies inequality (6.4) and hence inequality (6.2) if $n$ is large enough.

Now let us choose an $N$ so that inequality (6.2) holds for all $n>N$, and then let us choose a $c$ so that $p_{k}(n)<c n^{d n}$ holds for $n \leq N$. Then we show that $p_{k}(n)<c n^{d n}$ also holds for $n>N$ by induction on $n$. Suppose that we already know that $p_{k}(m)<c m^{d m}$ holds for all $m<n$. Then, by using the recursion formula (6.1) and inequality (6.2), we obtain

$$
\begin{aligned}
p_{k}(n) & =\sum_{i=0}^{k-1}\left(\begin{array}{c}
n-1 \\
i
\end{array}\right) p_{k}(n-1-i) \\
& <c \sum_{i=0}^{k-1}\left(\begin{array}{c}
n-1 \\
i
\end{array}\right)(n-1-i)^{d(n-1-i)}<c n^{d n} .
\end{aligned}
$$

\subsection{The number of $\nabla$-classes in point stabilisers}

In this section, we examine the possible growth of the number of $\nabla$-classes in stabilisers of finite sets.

Lemma 6.3. Let $G \in \mathcal{E}_{\exp }+$ be a permutation group on a countably infinite set $X$, that is, $o_{n}^{i}(G) \leq c_{1} n^{d n}$ for some $c_{1}, d$ with $d<1$. Let $F \subset X$ be finite. Then, for every $\varepsilon>0$,

- there exists a constant $c_{2}$ such that $o_{n}^{i}\left(\left.G_{F}\right|_{X \backslash F}\right)<c_{2} n^{(d+\varepsilon) n}$,

- there exists a constant $c_{3}$ such that $o_{n}^{i}\left(G_{F}\right)<c_{3} n^{(d+\varepsilon) n}$.

In particular, $G_{F} \in \mathcal{E}_{\exp +}$ and $\left.G_{F}\right|_{X \backslash F} \in \mathcal{E}_{\exp +}$.

Proof. Let $\varepsilon>0$. The orbits of injective $n$-tuples of $G_{F}$ can be embedded into the orbits of injective $(n+|F|)$-tuples of $G$ by mapping the orbit of a tuple $t$ into 
the orbit of $\left(t, t^{\prime}\right)$, where $t^{\prime}$ is any $|F|$-tuple such that $\left(t, t^{\prime}\right)$ has pairwise distinct entries and all elements of $F$ appear in $\left(t, t^{\prime}\right)$. Hence,

$$
o_{n}^{i}\left(G_{F}\right) \leq o_{n+|F|}^{i}(G) \leq c_{1}(n+|F|)^{d(n+|F|)} \leq c_{2} n^{(d+\varepsilon) n}
$$

for an appropriate constant $c_{2}$. Choosing $\varepsilon>0$ such that $d+\varepsilon<1$ shows that $G_{F} \in \mathcal{E}_{\exp +}$. The statements for $\left.G_{F}\right|_{X \backslash F}$ can be shown analogously.

Definition 6.4. Let $G$ be an oligomorphic permutation group on a countably infinite set $X$. For every finite set $F \subset X$, let $m_{G}(F)$ be the number of $\nabla\left(G_{F}\right)$ classes. For $n \in \mathbb{N}$, let $m_{G}(n):=\max \left(\left\{m_{G}(F)|F \subset X| F \mid,=n\right\}\right)$.

Remark 6.5. If $F_{1}, F_{2} \subset X$ are contained in the same orbit of $n$-subsets of $G$, then $m_{G}\left(F_{1}\right)=m_{G}\left(F_{2}\right)$. Hence, the set $\left\{m_{G}(F)|F \subset X| F \mid,=n\right\}$ is finite, and so the maximum of this set always exists.

Lemma 6.6. Let $G$ be a permutation group. Suppose that $o_{n}^{i}(G) \leq c_{1} n^{d n}$ for some $c_{1}$ and $d<1$. Then, for every $\varepsilon>0$, we have $m_{G}(n) \leq c_{2} n^{d+\varepsilon}$ for some constant $c_{2}$.

Proof. Suppose that $G$ is a permutation group on $X$, and let $F \subset X$ be of size $n$. Suppose that $X_{1}, X_{2}, \ldots, X_{l}$ are the infinite classes of the congruence $\nabla\left(G_{F}\right)$, and arbitrarily choose $x_{i} \in X_{i}$ for $i \in\{1, \ldots, l\}$. Then, for each function

$$
f:\{1, \ldots, k\} \rightarrow\{1, \ldots, l\}
$$

there are pairwise distinct elements $y_{1}, \ldots, y_{k}$ so that $y_{j} \in X_{f(j)} \backslash\left\{x_{f(j)}\right\}$. Let $t^{f}:=\left(x_{1}, \ldots, x_{l}, y_{1}, \ldots, y_{k}\right)$. Then the tuples $t^{f}$ are injective and lie in pairwise different orbits of $\left.G_{F}\right|_{X \backslash F}$. Thus, $l^{n} \leq o_{n}^{i}\left(\left.G_{F}\right|_{X \backslash F}\right) \leq c_{2} n^{(d+\varepsilon) n}$ for some $c_{2}$ by Lemma 6.3. Thus, $l \leq c_{2} n^{d+\varepsilon}$, and therefore $m_{G}(F) \leq c_{2} n^{d+\varepsilon}$.

Lemma 6.7. Let $G$ be a permutation group on a countably infinite set $X$, and suppose that $o_{n}^{i}(G) \leq \mathrm{cn}^{d n}$ for some $c$ and $d<1$. Let $F \subset X$ be finite, let $R$ be a congruence of $G_{F}$, and let $\frac{k-1}{k}>d$. Then $R$ has finitely many classes of size at least $k$.

Proof. By Lemma 6.3, we can assume that $F=\emptyset$. Suppose to the contrary that $R$ has infinitely many classes of size at least $k$. Let $n$ be arbitrary, and let $\mathcal{P}_{n}^{k}$ be the set of partitions $P=\left\{S_{1}, \ldots, S_{l}\right\}$ of $\{1,2, \ldots, n\}$ such that $\left|S_{i}\right| \leq k$ for all $i=1, \ldots, l$. For each $P \in \mathcal{P}_{n}^{k}$, choose pairwise distinct elements $x_{1}^{P}, \ldots, x_{n}^{P} \in X$ such that $x_{i}^{P} R x_{j}^{P}$ if and only if $x_{i}^{P}$ and $x_{j}^{P}$ are in the same set in the partition $P$. Then the $n$-tuples $\left(x_{1}^{P}, \ldots, x_{n}^{P}\right)$ for $P \in \mathcal{P}_{n}^{k}$ are injective and lie in pairwise 
different orbits of $G$. Therefore, $o_{n}^{i}(G) \geq\left|\mathcal{P}_{n}^{k}\right|$. Let us choose $\varepsilon>0$ such that $\frac{k-1}{k}-\varepsilon>d$. Then, by Lemma 6.1 , it follows that

$$
o_{n}^{i}(G) \geq\left|\mathcal{P}_{n}^{k}\right|=p_{k}(n) \geq n^{\left(\frac{k-1}{k}-\varepsilon\right) n}>c n^{d n}
$$

for $n$ large enough. This contradicts our assumption.

Corollary 6.8. Let $G$ be a permutation group on a countably infinite set $X$, and suppose that $o_{n}^{i}(G) \leq c n^{d n}$ for some $c$ and $d<1$. Let $F \subset X$ be finite, and let $R$ be a congruence of $G_{F}$. Then $R$ has finitely many infinite classes.

Proof. Follows directly from Lemma 6.7.

Definition 6.9. Let $\left(\mathcal{E}_{\exp +}\right)^{k}, k \in \mathbb{N}$, denote the class of those groups $G \in \mathcal{E}_{\exp +}$ for which the following holds.

For every finite $F \subset X$, every congruence of $G_{F}$ has at most finitely many equivalence classes of size at least $k$.

Let $\left(\mathcal{K}_{\exp +}\right)^{k}$ denote the classes of those structures in $\mathcal{K}_{\exp +}$ whose automorphism group is in $\left(\mathscr{E}_{\exp +}\right)^{k}$.

Using the definition above, Lemma 6.7 immediately implies the following.

Corollary 6.10. $\mathcal{E}_{\exp +}=\bigcup_{k=1}^{\infty}\left(\mathcal{E}_{\exp +}\right)^{k}$, and $\mathcal{K}_{\exp +}=\bigcup_{k=1}^{\infty}\left(\mathcal{K}_{\exp +}\right)^{k}$.

\subsection{The primitive case}

We use the following theorem of Dugald Macpherson [55].

Theorem 6.11 ([55, Theorem 1.2]). Let $G$ be a permutation group on a countably infinite set $X$ which is primitive but not highly transitive. Then there is a polynomial $p$ such that $o_{n}^{i}(G) \geq \frac{n !}{p(n)}$.

Theorem 6.11 immediately implies the following.

Lemma 6.12. Let $G \in \mathcal{E}_{\exp +}$ be primitive. Then $G$ is highly transitive.

Proof. Stirling's formula implies that, for all $c$ and $d<1$ and every polynomial $p$, if $n$ is large enough, then $\frac{n !}{p(n)}>c n^{d n}$. Hence, the lemma follows from Theorem 6.11 . 


\subsection{The case when $\Delta(G)$ is trivial}

The result of Macpherson (Theorem 6.11) is used via the following lemma.

Lemma 6.13. Let $G \in \mathcal{E}_{\exp +}$ be such that $\Delta(G)$ is trivial and such that $G$ stabilises each class of $\nabla(G)$ setwise. Then $G$ acts highly transitively on each of its orbits.

Proof. Let $O_{1}, \ldots, O_{m}$ be the orbits of $G$. Then $O_{1}, \ldots, O_{m}$ are also the classes of $\nabla(G)$. We claim that the action of $G$ on $O_{i}$ is primitive for each $i \in\{1, \ldots, m\}$; this suffices, because then the statement of the lemma follows from Lemma 6.12.

Let $R_{i}$ be a congruence of $\left.G\right|_{O_{i}}$. Since $G$ acts transitively on $O_{i}$, it follows that every class of $R_{i}$ has the same size. If this size is finite, then let us consider the congruence $R_{i}^{*}:=R_{i} \cup\left\{(x, x) \mid x \in X \backslash O_{i}\right\}$. Then every class of $R_{i}^{*}$ is finite and thus $R_{i}^{*}$ must be finer than $\Delta(G)$. Since $\Delta(G)$ is the identity congruence, $R_{i}^{*}$ and $R_{i}$ are the identity congruence, too.

Now assume that every class of $R_{i}$ is infinite. Then, by Corollary $6.8, R_{i}$ has finitely many classes. Let $C_{1}, C_{2}, \ldots, C_{l}$ be these classes. Then

$$
\left\{O_{1}, \ldots, O_{i-1}, O_{i+1}, \ldots, O_{m}, C_{1}, C_{2}, \ldots, C_{l}\right\}
$$

is an invariant partition. Since $\nabla(G)$ is the finest congruence with finitely many classes, it follows that $l=1$, and thus $R_{i}$ is again the identity congruence. Therefore, $\left.G\right|_{O_{i}}$ is primitive for all $i$.

Under the conditions of Lemma 6.13, we will show that, in fact, if $G$ is closed, then $G=\prod_{i=1}^{m} \operatorname{Sym}\left(O_{i}\right)$, where $O_{1}, \ldots, O_{m}$ are the orbits of $G$, that is, $G$ is the automorphism group of a unary structure (Lemma 6.22). The following lemma is well known (see, e.g., [58, Proposition 1.4 (2)] or [29, Corollary 2.2]).

Lemma 6.14. Every normal subgroup of a highly transitive permutation group acting on an infinite set is either highly transitive or trivial.

Lemma 6.15. Let $G$ be a closed permutation group on a countably infinite set $X$. Let $T$ be an infinite orbit of $G$ such that $\left.G\right|_{T}$ is highly transitive, and $S:=X \backslash T$. Then one of the following holds.

(1) $\left\{\operatorname{id}_{S}\right\} \times \operatorname{Sym}(T) \subseteq G$.

(2) There exists a surjective homomorphism e: $\left.\left.G\right|_{S} \rightarrow G\right|_{T}$ such that a permutation $\gamma$ of $X$ is in $G$ if and only if there exists a permutation $\left.\alpha \in G\right|_{S}$ so that $\left.\gamma\right|_{S}=\alpha$ and $\left.\gamma\right|_{T}=e(\alpha)$. 
Proof. If $\alpha \in \operatorname{Sym}(S)$ and $\beta \in \operatorname{Sym}(T)$, then we use the notation $(\alpha, \beta)$ for the unique permutation $\gamma \in \operatorname{Sym}(X)$ whose restriction to $S$ equals $\alpha$ and whose restriction to $T$ equals $\beta$.

Case 1. For every $\left.\alpha \in G\right|_{S}$, there is a unique $\left.e(\alpha) \in G\right|_{T}$ such that $(\alpha, e(\alpha)) \in G$. It is easy to see that, in this case, $e$ is a surjective homomorphism from $\left.G\right|_{S}$ to $\left.G\right|_{T}$; therefore condition (2) holds.

Case 2. For some $\left.\alpha \in G\right|_{S}$, there exist at least two distinct permutations $\beta_{1}, \beta_{2} \in$ $\left.G\right|_{T}$ such that $\gamma_{1}:=\left(\alpha, \beta_{1}\right) \in G$ and $\gamma_{2}:=\left(\alpha, \beta_{2}\right) \in G$.

Let $K:=\left\{\beta \in \operatorname{Sym}(T) \mid\left(\operatorname{id}_{S}, \beta\right) \in G\right\}$. Then

- $K$ is nontrivial since $\beta_{1} \beta_{2}^{-1} \in K$,

- $K$ is closed in $\operatorname{Sym}(T)$,

- $K$ is a normal subgroup of $\operatorname{Sym}(T)$.

To prove normality, let (id, $\beta) \in G$, and let $\delta \in \operatorname{Sym}(T)$ be arbitrary. Since $\left.G\right|_{T}$ is dense in $\operatorname{Sym}(T)$, there is a sequence $\delta_{1}, \delta_{2}, \ldots$ of elements of $\left.G\right|_{T}$ which converges to $\delta \in \operatorname{Sym}(T)$. By the definition of $\left.G\right|_{T}$, we know that there exist elements $\alpha_{i} \in \operatorname{Sym}(S)$ such that $\eta_{i}:=\left(\alpha_{i}, \delta_{i}\right) \in G$ for every $i \in \mathbb{N}$. Then

$$
G \ni \eta_{i}(\mathrm{id}, \beta) \eta_{i}^{-1}=\left(\mathrm{id}, \delta_{i} \beta \delta_{i}^{-1}\right) \text {. }
$$

Therefore, $\lim _{i}\left(\mathrm{id}, \delta_{i} \beta \delta_{i}^{-1}\right)=\left(\mathrm{id}, \delta \beta \delta^{-1}\right) \in G$ since $G$ is closed. By definition, this implies that $\delta \beta \delta^{-1} \in K$ which shows that $K$ is indeed a normal subgroup. By Lemma 6.14, $K=\operatorname{Sym}(T)$. Thus, $\left\{\operatorname{id}_{S}\right\} \times \operatorname{Sym}(T) \subseteq G$, i.e., condition (1) holds.

Lemma 6.16. Let $G$ be a closed oligomorphic permutation group on a countably infinite set $X$. Let $O_{1}, \ldots, O_{m}$ be the orbits of $G$. Let us suppose that each $O_{i}$ is infinite and that $G$ acts highly transitively on each $O_{i}$. Let $l \in\{1, \ldots, m\}$, and let $S:=\bigcup_{i=1}^{l} O_{i}$ be such that $\operatorname{acl}_{G}(S)=X$. Then $H:=\left.G\right|_{S}$ is closed in $\operatorname{Sym}(S)$.

Proof. We first show the statement for $l=m-1$. Let $T:=O_{m}$ (so that we have the same notation as in Lemma 6.15). First, let us assume that Lemma 6.15(1) holds. Let $\left(\alpha_{j}\right)_{j \in \mathbb{N}}$ be a sequence that converges in $\left.G\right|_{S}$ to $\alpha \in \operatorname{Sym}(S)$. Let $\beta_{j} \in\left\{\operatorname{id}_{S}\right\} \times \operatorname{Sym}(T) \subseteq G$ be such that $\left.\alpha_{j}\right|_{T}=\left.\beta_{j}\right|_{T}$, and let $\alpha_{j}^{\prime}:=\alpha_{j} \beta_{j}^{-1} \in G$. Then $\alpha_{j}^{\prime} \rightarrow(\alpha, \operatorname{id}(T)) \in G$ since $G$ is closed. In particular, $\left.\alpha \in G\right|_{S}$ and $H$ is closed.

Otherwise, if condition (1) of Lemma 6.15 does not hold, then by Lemma 6.15, we can assume that item (2) of Lemma 6.15 holds. Let $e:\left.\left.G\right|_{S} \rightarrow G\right|_{T}$ be as in 
item (2) of Lemma 6.15. If $F \subset S$ is finite and $\left.\alpha \in G\right|_{S}$, then

$$
\begin{aligned}
\operatorname{acl}(\alpha(F)) \cap T & =\operatorname{acl}((\alpha, e(\alpha))(F)) \cap T=(\alpha, e(\alpha))(\operatorname{acl}(F)) \cap T \\
& =(\alpha, e(\alpha))(\operatorname{acl}(F) \cap T)=e(\alpha)(\operatorname{acl}(F) \cap T) .
\end{aligned}
$$

By assumption, $\operatorname{acl}(F) \cap T$ is non-empty for some $F$ (and it is always finite). Let

$$
k:=|\operatorname{acl}(F) \cap T| .
$$

Then, by our previous observation and the fact that $\left.G\right|_{T}=e\left(\left.G\right|_{S}\right)$ is highly transitive, it follows that, for any subset $F^{\prime}$ of $T$ of size $k$, there exists a finite subset $F^{\prime \prime}$ of $S$ such that $\operatorname{acl}\left(F^{\prime \prime}\right) \cap T=F^{\prime}$.

We claim that, for all $x \in T$, there is a finite set $F$ of $S$ such that, for all $\alpha \in\left(\left.G\right|_{S}\right)_{F}$, it holds that $e(\alpha)(x)=x$. Let $F_{1}^{\prime}$ and $F_{2}^{\prime}$ be subsets of $T$ of size $k$ such that $F_{1}^{\prime} \cap F_{2}^{\prime}=\{x\}$. As we have seen, there exist finite subset $F_{1}^{\prime \prime}$ and $F_{2}^{\prime \prime}$ of $S$ such that $\operatorname{acl}\left(F_{i}^{\prime \prime}\right) \cap T=F_{i}^{\prime}$ for $i=1$ and $i=2$. Now let $F:=F_{1}^{\prime \prime} \cup F_{2}^{\prime \prime}$. Then if $\alpha \in\left(\left.G\right|_{S}\right)_{F}$, then $\alpha \in\left(\left.G\right|_{S}\right)_{F_{i}^{\prime \prime}}$, so $e(\alpha)\left(F_{i}^{\prime}\right)=F_{i}^{\prime}$. Therefore, we have $e(\alpha)(x) \in F_{1}^{\prime} \cap F_{2}^{\prime}=\{x\}$, that is $e(\alpha)(x)=x$.

Now let $\left(\alpha_{j}\right)_{j}$ be a convergent sequence in $\left.G\right|_{S}$. We want to show that the sequence $\left(e\left(\alpha_{j}\right)\right)_{j}$ is also convergent, i.e., for all $x \in T$, we have

$$
e\left(\alpha_{j}\right)(x)=e\left(\alpha_{j+1}\right)(x)
$$

if $j$ is large enough. By our claim, it follows that there is a finite set $F \subset S$ such that, for all $\alpha \in\left(\left.G\right|_{S}\right)_{F}$, we have $e(\alpha)(x)=x$. Since $\left(\alpha_{j}\right)_{j}$ is a convergent, there is an index $l$ such that $\alpha_{j}(y)=\alpha_{j+1}(y)$ for all $y \in F$ and $l \leq j$. Then if $j \geq l$, it follows that $\left.\alpha_{j} \alpha_{j+1}^{-1} \in H\right|_{F}$; hence $e\left(\alpha_{j}\right)\left(e\left(\alpha_{j+1}\right)\right)^{-1}(x)=e\left(\alpha_{j} \alpha_{j+1}^{-1}\right)(x)=x$, and thus $e\left(\alpha_{j}\right)(x)=e\left(\alpha_{j+1}\right)(x)$. Therefore, $\left(e\left(\alpha_{j}\right)\right)_{j}$ is convergent, which shows that $\left.G\right|_{S}$ is closed.

For $l<m-1$, note that $S \subseteq P:=O_{1} \cup \cdots \cup O_{m-1}$. Hence, $\operatorname{acl}(P)=X$, and we can apply the above argument for $P$ instead of $S$. We obtain that $\left.G\right|_{P}$ is closed. Hence, the group $\left.G\right|_{P}$ satisfies all the assumptions for $G$ but has fewer orbits, so by induction, we finally obtain that $\left.G\right|_{S}$ is closed.

In the proof of the next lemma, it will be convenient to use a recent general result of Paolini and Shelah. A closed subgroup $G$ of $\operatorname{Sym}(X)$ has the

- small index property if every subgroup of $G$ of index less than $2^{\aleph_{0}}$ is open, i.e., contains the pointwise stabiliser of a finite set $F \subset X$,

- strong small index property if every subgroup of $G$ of index less than $2^{\aleph_{0}}$ lies between the pointwise and the setwise stabiliser of a finite set $F \subset X$. 
The strong small index property of $\operatorname{Sym}(X)$ itself has been shown in [37]. (In fact, all automorphisms of $\omega$-categorical $\omega$-stable structures, and thus, by the results that we are about to prove, all groups in $\mathcal{E}_{\exp +}$, have the small index property [46].) On the other hand, already $R(U)$ contains structures whose automorphism groups do not have the strong small index property (take, e.g., an equivalence relation with two infinite classes). A permutation group $G$ on a set $X$ is said to have no algebraicity if $\operatorname{acl}_{G}(Y)=Y$ for every $Y \subseteq X$. The following has been proved in [60, Corollary 2].

Theorem 6.17 ([60]). Let $X_{1}$ and $X_{2}$ be countable, and let $G_{1} \leq \operatorname{Sym}\left(X_{1}\right)$ and $G_{2} \leq \operatorname{Sym}\left(X_{2}\right)$ be closed oligomorphic groups which have the strong small index property and have no algebraicity. Let us suppose that $\xi$ is a topological isomorphism from $G_{1}$ to $G_{2}$. Then there exists a bijection $b$ from $X_{1}$ to $X_{2}$ that induces $\xi$, i.e., for all $x \in X_{2}$ and $\alpha \in G_{1}$, we have

$$
(\xi \alpha)(x)=b\left(\alpha\left(b^{-1}(x)\right)\right) .
$$

The small index property for $G$ implies that, for a countable set $Y$, every homomorphism $h: G \rightarrow \operatorname{Sym}(Y)$ is continuous (this is easy to see and well known; see, e.g., [57, 62]). It follows from [43] that the image of a continuous homomorphism from $\operatorname{Sym}(X)$ to $\operatorname{Sym}(Y)$ is closed in $\operatorname{Sym}(Y)$ (see [7, Theorem 1.3] for a much more general recent result which also implies this).

Lemma 6.18. Let $G$ be a closed oligomorphic permutation group on a countably infinite set $X$. Let $O_{1}, \ldots, O_{m}$ be the orbits of $G$. Let us suppose that each $O_{i}$ is infinite, $G$ acts highly transitively on each $O_{i}$, and for some $l<m$ and $S:=\bigcup_{i=1}^{l} O_{i}$, we have $\operatorname{acl}_{G}(S)=X$ and $\left.G\right|_{S}=\operatorname{Sym}\left(O_{1}\right) \times \cdots \times \operatorname{Sym}\left(O_{l}\right)$. Then $\Delta(G)$ is not trivial.

Proof. Let $j \in\{l+1, \ldots, m\}$ and $T:=O_{j}$. Let $G_{j}:=\left.G\right|_{S \cup T}$. Then $G_{j}$ is closed by Lemma 6.16, and we can apply Lemma 6.15 to the group $G_{j}$ with respect to the partition of $S \cup T$ into $S$ and $T$. Since $T \subseteq \operatorname{acl}_{G}(S)$, it follows that condition (1) of Lemma 6.15 cannot hold. Thus, by Lemma 6.15, there exists a homomorphism $e_{j}:\left.G\right|_{S} \rightarrow \operatorname{Sym}\left(O_{j}\right)$ so that $G_{j}=\left\{\left(\alpha, e_{j}(\alpha)\right)|\alpha \in G|_{S}\right\}$. For $i \in\{1, \ldots, l\}$ and $\left.\alpha \in G\right|_{O_{i}}=\operatorname{Sym}\left(O_{i}\right)$, let $\hat{\alpha}_{i}$ denote the unique permutation of $S$ for which $\left.\hat{\alpha}_{i}\right|_{O_{i}}=\alpha$ and $\left.\hat{\alpha}_{i}\right|_{O_{k}}=\operatorname{id}_{O_{k}}$ if $k \neq i$. Then define the homomorphisms

$$
e_{i j}: \operatorname{Sym}\left(O_{i}\right) \rightarrow \operatorname{Sym}\left(O_{j}\right), \alpha \mapsto e_{j}\left(\hat{\alpha}_{i}\right) .
$$

As mentioned before the lemma, the map $e_{i j}$ is continuous. Let

$$
H_{i}:=\left\{\hat{\alpha}_{i} \mid \alpha \in \operatorname{Sym}\left(O_{i}\right)\right\}
$$


Then $\left.H_{i} \triangleleft G\right|_{S}$, and so $e_{j}\left(H_{i}\right) \triangleleft e_{j}\left(\left.G\right|_{S}\right)$. By definition, it follows that

$$
e_{j}\left(\left.G\right|_{S}\right)=\left.\left(G_{j}\right)\right|_{o_{j}}=\left.G\right|_{o_{j}} .
$$

In particular, $e_{j}\left(\left.G\right|_{S}\right) \leq \operatorname{Sym}\left(O_{j}\right)$ is highly transitive. Thus, by Lemma 6.14 , it follows that either $e_{j}\left(H_{i}\right)$ is also highly transitive or it is trivial. If $e_{i j}$ is trivial for every $i \in\{1, \ldots, l\}$, then $G$ fixes every element of $O_{j}$ contradicting the fact that $G$ acts transitively on $T=O_{j}$. Thus, there is an $i \in\{1, \ldots, l\}$ such that the image $I \leq \operatorname{Sym}\left(O_{j}\right)$ of $e_{i j}$ is highly transitive. As we have mentioned before the statement of the lemma, $I$ is a closed subset of $\operatorname{Sym}\left(O_{j}\right)$, so we can apply Theorem 6.17 and obtain a bijection $b_{j}$ between $O_{i}$ and $O_{j}$ which induces $e_{i j}$ (we could as well have derived this from the argument in [45, Example 2, page 224]). Now let $i^{\prime} \neq i$, and let $\alpha$ be a nontrivial permutation of $O_{i^{\prime}}$. Then $\hat{\alpha}_{i^{\prime}}$ commutes with every element of $H_{i}$, and so $e_{i^{\prime} j}(\alpha)$ commutes with every element of $e_{j}\left(H_{i}\right)=\operatorname{Sym}\left(O_{j}\right)$. Therefore, $e_{i^{\prime} j}(\alpha)=\operatorname{id}_{O_{j}}$. We have obtained that, for every $j \in\{l+1, \ldots, m\}$, there is a unique $i(j) \in\{1, \ldots, l\}$ and a bijection $b_{j}: O_{i(j)} \rightarrow O_{j}$ such that, for all $g \in G$ and $x \in O_{j}$, we have $g(x)=b_{j}\left(g\left(b_{j}^{-1}(x)\right)\right)$. In fact, the same is true in the case when $j \leq l$ : we can choose $i(j)$ to be $j$, and $b_{j}$ to be the identity map. Let $b$ be the union of the functions $b_{1}, \ldots, b_{m}$, and define the relation $\sim$ by $x \sim y \Leftrightarrow b(x)=b(y)$. Then $\sim$ is a congruence of $G$ all of whose classes are finite. Moreover, $\sim$ is nontrivial since $m>l$. This implies that also $\Delta(G)$ is nontrivial.

Lemma 6.19. Let $H$ be an oligomorphic permutation group on a countably infinite set $X$ with two infinite orbits $Y$ and $Z$. Let us assume that $H$ acts 2-transitively on $Z$ and that there exists $y \in Y$ so that $\left|\nabla\left(\left(H_{y}\right) \mid Z\right)\right| \geq 2$. Then, for every $n \in \mathbb{N}$, there exist $y_{1}, \ldots, y_{n} \in Y$ such that $\left|\nabla\left(\left(H_{y_{1}, \ldots, y_{n}}\right) \mid z\right)\right| \geq n+1$.

Proof. By transitivity, we know that, for all $y^{\prime} \in Y$, it holds that $\left|\nabla\left(H_{y^{\prime}} \mid z\right)\right| \geq 2$.

We show the statement of the lemma by induction on $n$. For $n=1$, the statement is trivial. Now suppose that we know there exist $y_{1}, \ldots, y_{n-1} \in Y$ such that $\nabla\left(\left(H_{\left.y_{1}, \ldots, y_{n-1}\right)}\right) \mid z\right)$ has at least $n$ classes. Let $C_{1}, \ldots, C_{m}$ be the classes of $\nabla\left(\left(H_{y_{1}, \ldots, y_{n-1}}\right) \mid z\right)$. Let $z_{1}, z_{2} \in C_{1}$. Since $H$ acts 2-transitively on $Z$, it follows that there exists a $y_{n} \in Y$ such that $\left(z_{1}, z_{2}\right) \notin \nabla\left(H_{y_{n}} \mid z\right)$. Let

$$
R:=\nabla\left(\left.\left(H_{y_{1}, \ldots, y_{n-1}}\right)\right|_{Z}\right) \cap \nabla\left(H_{y_{n}} \mid z\right) .
$$

Then $R$ is a congruence of $H_{y_{1}, \ldots, y_{n}}$ which is strictly finer than $\left(H_{y_{1}, \ldots, y_{n-1}, y_{n}}\right)$ and has finitely many classes. Therefore, we have that $\nabla\left(\left.\left(H_{y_{1}, \ldots, y_{n}}\right)\right|_{z}\right)$ is also finer than $\nabla\left(\left.\left(H_{y_{1}, \ldots, y_{n-1}}\right)\right|_{z}\right)$. In particular,

$$
\left|\nabla\left(\left.\left(H_{y_{1}, \ldots, y_{n}}\right)\right|_{z}\right)\right| \geq m+1 \geq n+1 .
$$


Lemma 6.20. Let $G \in \mathcal{E}_{\exp }+$ be closed. Suppose that $\Delta(G)$ is trivial and that $G$ stabilises each class of $\nabla(G)$ setwise. Let $O_{1}, \ldots, O_{l}$ be the orbits of $G$. Suppose that each $O_{i}$ is infinite and that $S:=X \backslash O_{l}$ is algebraically closed. Then $\left\{\operatorname{id}_{S}\right\} \times \operatorname{Sym}\left(O_{l}\right) \subseteq G$.

Proof. By our assumptions, the orbits $O_{1}, \ldots, O_{l}$ are the classes of $\nabla(G)$. By Lemma 6.13, it follows that $G$ acts highly transitively on each orbit $O_{i}$. We apply Lemma 6.15 to $S$ and $T:=O_{l}$. If item (1) of Lemma 6.15 applies, then we are done. We claim that item (2) of Lemma 6.15 cannot hold. For this, it suffices to show that $G$ contains a nontrivial permutation $\gamma$ such that $\left.\gamma\right|_{S}=\mathrm{id}_{S}$. Indeed, if there exists a homomorphism $e:\left.\left.G\right|_{S} \rightarrow G\right|_{T}$ as in item (2) of Lemma 6.15, then (using the notation from the proof of Lemma 6.15) $\gamma=\left(\operatorname{id}_{S}, e\left(\operatorname{id}_{S}\right)\right)=\left(\operatorname{id}_{S}, \mathrm{id}_{T}\right)$ is trivial.

Claim 1. For every finite $F \subseteq S$ and $L:=\left.G_{F}\right|_{T}$, the congruence $\nabla(L / \Delta(L))$ is universal.

Proof of Claim 1. Suppose to the contrary that there exists a finite $F \subseteq S$ such that $\nabla(L / \Delta(L))$ is not universal; choose a minimal $F$ with this property. For some $y \in F$, put $F^{\prime}:=F \backslash\{y\}$ and

$$
E:=\left.G_{F^{\prime}}\right|_{T}, \quad Z:=T / \Delta(E), \quad \text { and } \quad K:=E / \Delta(E) \leq \operatorname{Sym}(Z) .
$$

Let us consider the mapping $\pi: T \rightarrow Z$ which maps each element to its $\Delta(E)$ class. Then if $u, v \in Z^{n}$ are in different orbits of $K$, then all tuples in $\pi^{-1}(u)$ are in different orbits of $G$ than the tuples from $\pi^{-1}(v)$. Moreover, if $u \in Z^{n}$ is injective, then so are all the tuples in $\pi^{-1}(u)$. This means that the number of injective $n$-orbits of $K$ is at most $o_{n}^{i}\left(G_{F^{\prime}}\right)$. By Corollary 6.3, it follows that $o_{n}^{i}\left(G_{F^{\prime}}\right) \leq c n^{d n}$ for some constants $c, d$ with $d<1$. Therefore, $o_{n}^{i}(K) \leq c n^{d n}$, and thus $K \in \mathcal{E}_{\exp +}$. From the definition of $K$, it is clear that $\Delta(K)$ is the identity congruence. It follows from the minimal choice of $F$ that the congruence $\nabla(K)$ is the universal congruence. Therefore, Lemma 6.13 implies that $K$ is highly transitive.

Now let $Y:=O_{j} \backslash F^{\prime}$, where $j$ is such that $y \in O_{j}$. Then $G_{F^{\prime}}$ acts naturally on $Y \sqcup Z$. Let $H$ be the image of this action (as a subgroup of $\operatorname{Sym}(Y \sqcup Z)$ ); note that $H$ is highly transitive on $Z$. We claim that the group $H$, the orbits $Y, Z$ and the element $y \in Y$ satisfy the conditions of Lemma 6.19. The only nontrivial fact that we have to check is that the congruence $\nabla\left(\left.H_{y}\right|_{Z}\right)$ is not universal. We know that $\nabla(L / \Delta(L))$ is not universal. By Lemma 2.15, this is equivalent to the fact that the congruence generated by $\nabla(L)$ and $\Delta(L)$ is not universal. The congruence $\Delta(E)$ is also a congruence of $L$ with finite classes; hence $\Delta(E)$ is 
finer than $\Delta(L)$. Hence, the congruence generated by $\nabla(L)$ and $\Delta(E)$ is finer than the congruence generated by $\nabla(L)$ and $\Delta(L)$, and thus it is also not universal. Using Lemma 2.15 again, it follows that $\nabla(L / \Delta(E))$ is not universal. Since $L=\left.\left(G_{F^{\prime}}\right)_{y}\right|_{T}$, this means that the congruence $\nabla\left(\left.H_{y}\right|_{Z}\right)$ is not universal.

If we apply Lemma 6.19, we obtain that, for every $n$, there exist $y_{1}, \ldots, y_{n} \in Y$ so that $\left|\nabla\left(H_{y_{1}, \ldots, y_{n}} \mid z\right)\right| \geq n+1$. This also implies that

$$
\left|\nabla\left(G_{F^{\prime} \cup\left\{y_{1}, \ldots, y_{n}\right\}}\right)\right| \geq n+1,
$$

i.e., $m_{G}\left(F^{\prime} \cup\left\{y_{1}, \ldots, y_{n}\right\}\right) \geq n+1$. In particular, $m_{G}\left(\left|F^{\prime}\right|+n\right) \geq n+1$. This contradicts Lemma 6.6 for $0<\varepsilon<1-d$ if $n$ is large enough.

Claim 1 implies that, for every finite $F \subseteq S$, the group $G_{F}$ acts transitively on $T / \Delta\left(\left.G_{F}\right|_{T}\right)$. This also implies that all $\Delta\left(G_{F}\right)$-classes contained in $T$ have the same size. For a finite set $F \subset S$, let $k(F)$ denote this size. By Corollary 6.10, we have $G \in\left(\mathcal{E}_{\exp +}\right)^{k}$ for some $k$. This implies that $k(F) \leq k$ for every finite subset $F$ of $S$. This also implies that there exists a finite set $F$ so that $k(F)$ is maximal. So let us choose $F \subset S$ so that $k(F)$ is maximal, and similarly to above, let $L:=\left.G_{F}\right|_{T}, Z:=T / \Delta(L)$, and let $\pi: T \rightarrow Z$ map each element to its $\Delta(L)$ class.

Claim 2. For any finite $F^{\prime} \subset S$ that contains $F$, the group $G_{F^{\prime}}$ acts highly transitively on $Z$.

Proof of Claim 2. Let $E:=\left.G_{F^{\prime}}\right|_{T}$, and let $K:=E / \Delta(E)$. By the maximality of $k(F)$, it follows that $\Delta\left(G_{F}\right)$ and $\Delta\left(G_{F^{\prime}}\right)$ agree on $T$; hence $T / \Delta(E)=T / \Delta(L)$, and thus $K \leq \operatorname{Sym}(Z)$. As in the proof of Claim 1, it follows that $K \in \mathcal{E}_{\text {exp }+ \text {, and }}$ Claim 1 implies that $\nabla(K)$ is the universal congruence. So it is enough to show that $\Delta(K)$ is the identity congruence. In order to show this, let us consider the relation on $X$,

$$
R:=\left\{(x, y) \in T^{2} \mid(\pi(x), \pi(y)) \in \Delta(K)\right\} \cup\{(x, x) \mid x \in S\} .
$$

Then $R$ is a congruence of $G_{F^{\prime}}$ with finite classes. Therefore, $R$ is finer than $\Delta\left(G_{F^{\prime}}\right)$. As $\Delta\left(G_{F}\right)$ and $\Delta\left(G_{F^{\prime}}\right)$ agree on $T$, this is only possible if $\Delta(K)$ is the identity congruence. Hence, the conditions of Lemma 6.13 hold for $K$, and thus, by Lemma 6.13 , it follows that $K$ is highly transitive.

Now let us choose a prime $p>k(F)$, and let $z_{1}, \ldots, z_{p} \in Z$. Let

$$
F=S_{0} \subset S_{1} \subset \cdots \quad \text { and } \pi^{-1}\left(z_{1}\right) \cup \cdots \cup \pi^{-1}\left(z_{p}\right)=T_{0} \subset T_{1} \subset \cdots
$$


be sequences of finite subsets of $S$ and $T$, respectively, so that $\bigcup S_{i}=S$ and $\cup T_{i}=T$. By Claim 2, the stabiliser $G_{S_{i}}$ acts highly transitively on $Z=T / \Delta(L)$. In particular, there is a permutation $\gamma_{i}^{\prime} \in G$ which fixes every element in $S_{i}$ and which acts on $T_{i} / \Delta(L)$ as $\left(z_{1} z_{2} \ldots z_{p}\right)$. Now let $\gamma_{i}:=\left(\gamma_{i}^{\prime}\right)^{k(F) !}$. Then $\left.\gamma_{i}\right|_{T_{0}}$ is nontrivial, but $\left.\gamma_{i}\right|_{T_{i} \backslash T_{0}}=\mathrm{id}_{T_{i} \backslash T_{0}}$.

Since the permutations $\gamma_{i}$ have finitely many possible actions on the set $T_{0}$, we can assume, by choosing a subsequence if necessary, that $\left.\gamma_{i}\right|_{T_{0}}$ are the same for all $i$. Then the permutations $\gamma_{i}$ converge to a permutation $\gamma$ for which $\left.\gamma\right|_{S \cup T \backslash T_{0}}$ is trivial, but $\left.\gamma\right|_{T_{0}}$ is not trivial. Since $G$ is closed, it follows that $\gamma \in G$. This finishes the proof of the lemma.

Corollary 6.21. Let $G \in \mathcal{E}_{\exp }+$ be closed. Suppose that $\Delta(G)$ is trivial and that $G$ stabilises every class of $\nabla(G)$ setwise. Let $O_{1}, \ldots, O_{l}$ be the orbits of $G$. Suppose that each $O_{i}$ is infinite and that $X \backslash O_{i}$ is algebraically closed. Then $G=\operatorname{Sym}\left(O_{1}\right) \times \cdots \times \operatorname{Sym}\left(O_{l}\right)$.

Proof. Direct consequence of Lemma 6.20.

Lemma 6.22. Let $G \in \mathcal{E}_{\exp +}$ be closed and such that $\Delta(G)$ is trivial. Suppose that $G$ fixes every class of $\nabla(G)$ setwise. Let $O_{1}, \ldots, O_{m}$ be the orbits of $G$, and suppose that each $O_{i}$ is infinite. Then $G=\operatorname{Sym}\left(O_{1}\right) \times \cdots \times \operatorname{Sym}\left(O_{m}\right)$.

Proof. Let $I \subseteq\{1, \ldots, m\}$ be minimal so that $\operatorname{acl}_{G}(S)=X$ for $S:=\bigcup_{i \in I} O_{i}$. Without loss of generality, we can assume that $I=\{1, \ldots, l\}$ for some $l \leq m$. By Lemma 6.16, the group $\left.G\right|_{S} \in \mathscr{E}_{\exp +}$ is closed. By the minimality of $I$, it follows that the sets $S \backslash O_{i}$, for $i \in\{1, \ldots, l\}$, are algebraically closed with respect to $G$. By Corollary 6.21, it follows that $\left.G\right|_{S}=\operatorname{Sym}\left(O_{1}\right) \times \cdots \times \operatorname{Sym}\left(O_{l}\right)$. By Lemma 6.13, it follows that $G$ acts highly transitively on each orbit $O_{i}$. Thus, if $l<m$, then Lemma 6.18 implies that $\Delta(G)$ is nontrivial. This means that $l=m$, and thus $G=\left.G\right|_{S}=\operatorname{Sym}\left(O_{1}\right) \times \cdots \times \operatorname{Sym}\left(O_{m}\right)$.

In order to drop the condition that $G$ fixes every $\nabla(G)$-class, we need the following observation about finite index subgroups of oligomorphic groups.

Proposition 6.23. Let $G$ be an oligomorphic permutation group on a countably infinite set $X$, and let $H$ be a finite-index subgroup of $G$. Then

$$
o_{n}^{i}(H) \leq[G: H] \cdot o_{n}^{i}(G) .
$$

In particular, $H$ is oligomorphic. 
Proof. Choose elements $\gamma_{1}, \ldots, \gamma_{[G: H]} \in G$ such that $G=\bigcup_{i=1}^{[G: H]} \gamma_{i} H$. If the tuples $t_{1}, t_{2}, \ldots, t_{l}$ represent all injective $n$-orbits of $G$, then the tuples $\gamma_{i} t_{j}$ for $1 \leq i \leq[G: H]$ and $1 \leq j \leq l$ represent all injective $n$-orbits of $H$. Therefore, $o_{n}^{i}(H) \leq[G: H] \cdot o_{n}^{i}(G)$.

Lemma 6.24. Let $G \leq \operatorname{Sym}(X)$ be an oligomorphic permutation group, and let $H$ be a finite-index subgroup of $G$. Then $\operatorname{acl}_{H}(x) \subseteq \operatorname{acl}_{G}(x)$ for all $x \in X$.

Proof. By Proposition 6.23, the permutation group $H$ is oligomorphic. Let $x \in X$. We claim that the index $k:=\left[G_{x}: H_{x}\right]$ is finite. Let $K:=\bigcap_{g \in G} g^{-1} H g$, that is, $K$ is the kernel of the left action of $G$ on the left cosets of $H$ in $G$. Then $K \leq H$, and $|G / K|=[G: K]$ is finite. Then, by the second isomorphism theorem, we have

$$
G / K \supseteq G_{x} K / K \simeq G_{x} /\left(K \cap G_{x}\right)=G_{x} / K_{x} .
$$

In particular, $\left[G_{x}: K_{x}\right]=\left|G_{x} / K_{x}\right|$ is finite. Since $K \leq H$, we have $K_{x} \leq H_{x}$. Therefore, $\left[G_{x}: H_{x}\right]$ is also finite.

We have shown that $k:=\left[G_{x}: H_{x}\right]$ is finite. Choose elements

$$
\gamma_{1}, \ldots, \gamma_{k} \in \operatorname{Aut}(\mathfrak{U}) \text { such that } G_{x}=\bigcup_{i=1}^{k} \gamma_{i} H_{x} .
$$

Let $y \in \operatorname{acl}_{H}(x)$. Since $\left[H: H_{x}\right]$ is finite, $H_{x}(y)$ is finite. Therefore,

$$
G_{x}(y)=\left(\bigcup_{i=1}^{k} \gamma_{i} H_{x}\right)(y)=\bigcup_{i=1}^{k} \gamma_{i} H_{x}(y)
$$

is finite, that is, $y \in \operatorname{acl}_{G}(x)$. This proves that $\operatorname{acl}_{H}(x) \subseteq \operatorname{acl}_{G}(x)$.

Lemma 6.25. Let $G$ be an oligomorphic permutation group on a countably infinite set $X$, and let $H$ be a finite-index subgroup of $G$. Then $\Delta(G)=\Delta(H)$.

Proof. Clearly, $\Delta(G)$ is a congruence of $H$ with finite classes. Thus, we have $\Delta(G) \subseteq \Delta(H)$. Now let $(x, y) \in \Delta(H)$. Then $y \in \operatorname{acl}_{H}(x)$ and $x \in \operatorname{acl}_{H}(y)$ by Lemma 2.13. By Lemma 6.24, this implies that $y \in \operatorname{acl}_{G}(x)$ and $x \in \operatorname{acl}_{G}(y)$. Again by Lemma 2.13, we obtain $(x, y) \in \Delta(G)$, showing that $\Delta(G)=\Delta(H)$.

Theorem 6.26. Let $G \in \mathcal{E}_{\exp +}$ be closed such that $\Delta(G)$ is trivial. Let $O_{1}, \ldots, O_{m}$ be the classes of $\nabla(G)$. Then $\operatorname{Sym}\left(O_{1}\right) \times \cdots \times \operatorname{Sym}\left(O_{m}\right) \subseteq G$.

Proof of Theorem 6.26. Let $K$ be the kernel of the action of $G$ on $\left\{O_{1}, \ldots, O_{m}\right\}$. Then $[G: K]$ is finite, and thus, by Proposition 6.23, it follows that $K \in \mathscr{E}_{\exp +}$. 
Without loss of generality, we can assume that $O_{1}, \ldots, O_{l}$ are the infinite orbits of $K$. Let $Y=O_{1} \cup \cdots \cup O_{l}$. Then $X \backslash Y$ is finite, and $K$ fixes each element in

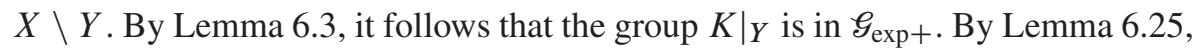
it follows that $\Delta(K)$ is trivial, and thus $\Delta\left(\left.K\right|_{Y}\right)$ is also trivial. Moreover, $\left.K\right|_{Y}$ fixes every class of $\nabla\left(\left.K\right|_{Y}\right)$ setwise, and all orbits of $\left.K\right|_{Y}$ are infinite. Hence, we can apply Lemma 6.22 and obtain that $\left.K\right|_{Y}=\operatorname{Sym}\left(O_{1}\right) \times \cdots \times \operatorname{Sym}\left(O_{l}\right)$. Therefore, $\operatorname{Sym}\left(O_{1}\right) \times \cdots \times \operatorname{Sym}\left(O_{m}\right)=K \subseteq G$.

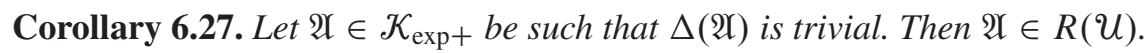

Proof. Apply Theorem 6.26 to Aut( $(\mathfrak{U})$, and combine with Corollary 3.10.

\subsection{The general case}

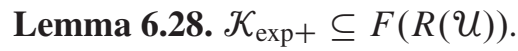

Proof. Let $\mathfrak{U} \in \mathcal{K}_{\exp +}$, and let us consider the factor mapping $\pi: \mathfrak{U} \rightarrow \mathfrak{B}$, where $\mathfrak{B}:=\mathfrak{U} / \Delta(\mathfrak{H})$. Recall that, by $\mathfrak{U} / \Delta(\mathfrak{H})$, we denote any structure with

$$
\operatorname{Aut}(\mathfrak{U} / \Delta(\mathfrak{U}))=\operatorname{Aut}(\mathfrak{U}) / \Delta(\mathfrak{U}) .
$$

If $u, v \in B$ are in different orbits of Aut( $(\mathfrak{B})$, then the tuples in $\pi^{-1}(u)$ lie in different orbits of Aut $(\mathfrak{N})$ than the tuples in $\pi^{-1}(v)$. Moreover, if $u \in B^{n}$ is injective, then so are the tuples in $\pi^{-1}(u)$. This means that the number of injective $n$-orbits

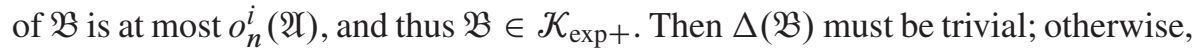
Aut $(\mathfrak{B})$ has a nontrivial congruence all of whose classes are finite, contradicting the definition of $\Delta(\mathfrak{V})$. By Corollary 6.27, it then follows that $\mathfrak{B} \in R(\mathcal{U})$, and thus $\mathfrak{U} \in F(R(\mathcal{U}))$.

The reverse containment holds as well.

Theorem 6.29. $\mathcal{K}_{\exp +}=F(R(U))=R^{<\infty}\left(F\left(U^{*}\right)\right)$.

Proof. We already know that

$$
\begin{aligned}
\mathcal{K}_{\exp +} & \subseteq F(R(\mathcal{U})) & & (\text { Lemma 6.28), } \\
F(R(U)) & \subseteq R^{<\infty}\left(F\left(\mathcal{U}^{*}\right)\right) & & (\text { see Remark 5.8). }
\end{aligned}
$$

So we have to show that $R^{<\infty}\left(F\left(U^{*}\right)\right) \subseteq \mathcal{K}_{\exp +}$. Proposition 2.33 implies that $R^{<\infty}\left(\mathcal{K}_{\exp +}\right)=\mathcal{K}_{\exp +}$. Therefore, it is enough to show that $F\left(U^{*}\right) \subseteq \mathcal{K}_{\exp +}$. So let $\mathfrak{B} \in \mathcal{U}^{*}$, and let $\pi: \mathfrak{U} \rightarrow \mathfrak{B}$ be a finite covering. Lemma 4.9 shows that $\pi$ is strongly split. Therefore, we can assume that $\pi$ is a strongly trivial covering map. 
It follows from the description of trivial coverings given in Remark 4.11 that the orbit of an injective $n$-tuple $t=\left(t_{1}, \ldots, t_{n}\right)$ of a trivial covering of a unary structure is uniquely determined by the orbits of $t_{1}, \ldots, t_{n}$ and by the partition of the set $\left\{t_{1}, \ldots, t_{n}\right\}$ defined by the congruence $\sim_{\pi}$. This means that the number of injective orbits of $\mathfrak{U}$ it at most $m^{n} \cdot p_{k}(n)$, where

- $m$ is the number of orbits of Aut( $(\mathfrak{U})$,

- $k$ is the maximal size of the classes of $\sim_{\pi}$,

- $p_{k}(n)$ is the number of partitions of $\{1, \ldots, n\}$ with parts of size at most $k$ (see Section 6.1).

Choose $d>\frac{k-1}{k}$. Then, by Lemma 6.2, we have $p_{k}(n)<c_{1} n^{d n}$ for some $c_{1}$. Thus, $o_{n}^{i}(\mathfrak{U}) \leq m^{n} c_{1} n^{d n} \leq c_{2} n^{d n}$ for some $c_{2}$. Therefore, $\mathfrak{U} \in \mathcal{K}_{\exp +\text {. }}$

Remark 6.30. Recall from Proposition 4.9 that every finite cover $\pi: \mathfrak{A} \rightarrow \mathfrak{B}$ for $\mathfrak{B} \in U^{*}$ is strongly split, and hence all structures in $\mathcal{K}_{\exp +}=R\left(F\left(U^{*}\right)\right)$ have a first-order interpretation in $(\mathbb{N} ;=)$ (Remark 4.12). Since $(\mathbb{N} ;=)$ is $\omega$-stable and first-order interpretations preserve $\omega$-stability, it follows that all structures in $\mathcal{K}_{\text {exp}}+$ are $\omega$-stable.

\subsection{Thomas' conjecture for the class $\mathcal{K}_{\text {exp+ }}$}

Let $k, m \in \mathbb{N}$. Let $\mathscr{G}(k, m)$ be the class of all oligomorphic permutation groups $G$ for which the classes of $\Delta(G)$ have size at most $k$ and where $\nabla(G / \Delta(G))$ has at most $m$ classes. Let $\delta(k, m)$ be the class of all structures whose automorphism group is in $\mathcal{E}(k, m)$.

Lemma 6.31. Let $k, m \in \mathbb{N}$. Let $\mathfrak{B} \in \mathcal{U}^{*}$, let $\pi: \mathfrak{U} \rightarrow \mathfrak{B}$ be a finite covering, and let $\mathfrak{C}$ be a quasi-covering reduct of $\mathfrak{B}$. Then $\mathfrak{C} \in \mathcal{S}(k, m)$ if and only if $\mathfrak{U} \in \delta(k, m)$.

Proof. By definition, $\Delta(\mathfrak{U})=\Delta(\mathfrak{C})=\sim_{\pi}$, and

$$
\nabla(\operatorname{Aut}(\mathfrak{C} / \Delta(\mathfrak{C})))=\nabla(\operatorname{Aut}(\mathfrak{C} / \Delta(\mathfrak{U})))=\nabla(\operatorname{Aut}(\mathfrak{U} / \Delta(\mathfrak{U})))=\nabla(\mathfrak{B}) .
$$

Lemma 6.32. Let $k, m \in \mathbb{N}$. There are finitely many structures in $\mathcal{K}_{\exp }+\cap 8(k, m)$ up to bi-definability.

Proof. By Theorem 6.29, we know that $\mathcal{K}_{\exp +}=(F \circ R)(\mathcal{U})$. Proposition 5.11 implies that every structure $\mathfrak{C}$ in $\mathcal{K}_{\exp +}$ is a quasi-covering reduct of a finite covering structure $\mathfrak{A}$ of some structure in $\mathcal{U}^{*}$. By Lemma 6.31, we know that 
$\mathfrak{C} \in \mathcal{S}(k, m)$ if and only if $\mathfrak{A} \in \mathcal{S}(k, m)$. If $\mathfrak{A}$ is a trivial covering of some structure in $U^{*}$, then by Theorem 5.10 , it has finitely many quasi-covering reducts. Therefore, it is enough to show that there are finitely many structures in $\delta(k, m)$ up to bi-definability which are strongly trivial covering structures of some structure in $U^{*}$.

Let $\mathfrak{B} \in \mathcal{U}^{*}$, and let $\pi: \mathfrak{A} \rightarrow \mathfrak{B}$ be a strongly trivial finite covering map. Let $O_{1}, \ldots, O_{l}$ be the orbits of $\mathfrak{B}$. Then $l \leq m$. Following Remark 4.11, we can assume without loss of generality that $A=\bigsqcup_{i=1}^{l} F_{i} \times O_{i}$ for some finite sets $F_{i}$, and

$$
\operatorname{Aut}(\mathfrak{U})=\bigsqcup_{i=1}^{l} \operatorname{id}_{F_{i}} \prec \operatorname{Sym}\left(O_{i}\right) .
$$

Since $\sim_{\pi}$ is a congruence with finite classes, it follows that $\left|F_{i}\right| \leq k$. Then there are finitely many options for $l$, the sizes of the orbits $O_{i}$ (they are all either one or infinite), and the sizes of the sets $F_{i}$, and if we fix these parameters, then the group $\operatorname{Aut}(\mathfrak{Q})$ is uniquely determined up to isomorphism. This implies that there are finitely many structures in $\delta(k, m)$ up to bi-definability which are a trivial covering structure of a structure in $\mathcal{U}^{*}$.

Lemma 6.33. Let $\mathfrak{A} \in \mathcal{K}_{\exp +}$, and let $\mathfrak{B} \in R(\mathfrak{U})$. Let $k$ be the size of the largest $\Delta(\mathfrak{U})$-class, and let $m$ be the number of $\nabla(\mathfrak{U})$-classes. Then $\mathfrak{B} \in \mathcal{S}(k, m)$.

Proof. If $R$ is a congruence of $\operatorname{Aut}(\mathfrak{B})$, then it is also a congruence of $\operatorname{Aut}(\mathfrak{Q})$. Therefore, the size of every class of $\Delta(\mathfrak{B})$ is at most $k$. Similarly, the number of $\nabla(\mathfrak{B})$-classes is at most the number of $\nabla(\mathfrak{U})$-classes. The number of $\nabla(\mathfrak{B})$ classes is an upper bound for the number of $\nabla(\mathfrak{B} / \Delta(\mathfrak{B}))$-classes. This proves the lemma.

Lemma 6.32 and Lemma 6.33 immediately imply the following weak version of Thomas' conjecture for the class $\mathcal{K}_{\exp +}$.

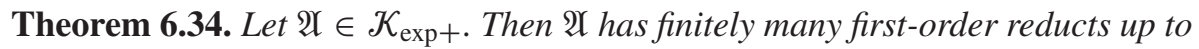
bi-definability.

Theorem 6.34 implies the (standard version of) Thomas' conjecture as follows. First we state an important well-known link between infinite descending chains of first-order reducts and infinite signatures. We say that a structure $\mathfrak{B}$ has essentially infinite signature if there does not exist a structure $\mathfrak{B}^{\prime}$ with finite signature such that $\operatorname{Aut}(\mathfrak{B})=\operatorname{Aut}\left(\mathfrak{B}^{\prime}\right)$. 
Lemma 6.35. Let $\mathfrak{A}$ be an $\omega$-categorical structure. Then there exists an infinite sequence $\mathfrak{B}_{1}, \mathfrak{B}_{2}, \ldots$ of first-order reducts of $\mathfrak{A}$ such that $\operatorname{Aut}\left(\mathfrak{B}_{1}\right) \supsetneq \operatorname{Aut}\left(\mathfrak{B}_{2}\right) \supsetneq \ldots$ if and only if $\mathfrak{Q}$ has a reduct with essentially infinite signature.

Proof. Assume that the reduct $\mathfrak{B}=\left(B ; R_{1}, R_{2}, \ldots\right)$ of $\mathfrak{U}$ has essentially infinite signature. By assumption, $\mathfrak{B}$ and $\mathfrak{B}_{n}:=\left(B ; R_{1}, \ldots, R_{n}\right)$ are not first-order interdefinable. Moreover, for every $n \in \mathbb{N}$, there exists an $f(n) \in \mathbb{N}$ such that $\mathfrak{B}_{n}$ and $\mathfrak{B}_{f(n)}$ are not first-order interdefinable (otherwise, every relation in $\mathfrak{B}$ would be first-order definable in $\mathfrak{B}_{n}$, contradicting our assumptions). So

$$
\mathfrak{B}_{1}, \mathfrak{B}_{f(1)}, \mathfrak{B}_{f(f(1))}, \ldots
$$

provides an infinite strictly descending chain of first-order reducts of $\mathfrak{A}$.

Suppose conversely that $\mathfrak{B}_{1}, \mathfrak{B}_{2}, \ldots$ is an infinite strictly descending chain of first-order reducts of $\mathfrak{A}$. Define $\mathfrak{C}$ as the first-order reduct of $\mathfrak{U}$ whose relations are precisely the relations of all the $\mathfrak{B}_{i}$. Assume for contradiction that there exists a finite-signature structure $\mathfrak{C}^{\prime}$ with $\operatorname{Aut}\left(\mathfrak{C}^{\prime}\right)=\operatorname{Aut}(\mathfrak{C})$. Let $i \in \mathbb{N}$ be such that all relations used in the definitions of the relations of $\mathbb{E}^{\prime}$ in $\mathbb{E}$ already appear in the signature of $\mathfrak{B}_{i}$. Then $\operatorname{Aut}\left(\mathfrak{B}_{i}\right)=\operatorname{Aut}\left(\mathfrak{C}^{\prime}\right)=\operatorname{Aut}(\mathfrak{C})=\operatorname{Aut}\left(\mathfrak{B}_{j}\right)$ for all $j \geq i$, contradicting the assumption that $\left(\mathfrak{B}_{i}\right)_{i \in \mathbb{N}}$ is strictly decreasing.

Proposition 6.36. Let $\mathfrak{2}$ be an $\omega$-categorical structure. Then $\mathfrak{A}$ has finitely many first-order reducts up to interdefinability if and only if $\mathfrak{X}$ has finitely many firstorder reducts up to bidefinability.

Proof. If $\mathfrak{B}$ is a first-order reduct of $\mathfrak{A}$ with essentially infinite signature, then $\mathfrak{B}$ has an infinite strictly descending chain of first-order reducts. Note that if $\operatorname{Aut}\left(\mathfrak{B}_{1}\right) \subsetneq \operatorname{Aut}\left(\mathfrak{B}_{2}\right)$, then for some $n$, there are strictly more orbits of $n$-tuples in $\operatorname{Aut}\left(\mathfrak{B}_{1}\right)$ than in $\operatorname{Aut}\left(\mathfrak{B}_{2}\right)$, so $\mathfrak{B}_{1}$ and $\mathfrak{B}_{2}$ are not bidefinable (if two reducts are bidefinable, then they have the same number of orbits of $n$-tuples for all $n$ ). So $\mathfrak{B}$ and $\mathfrak{A}$ have infinitely many first-order reducts up to bi-definability, so the statement is trivially true in this case.

Therefore, it suffices to show that every first-order reduct $\mathfrak{B}$ of $\mathfrak{U}$ with finite signature is bidefinable to at most finitely many reducts of $\mathfrak{B}$ up to interdefinability. The equivalence class of $\mathfrak{B}$ with respect to interdefinability is given by its orbits of $n$-tuples, for some finite $n$ (since $\mathfrak{B}$ has finite signature), and thus the same holds for any structure which is bidefinable $\mathfrak{B}$. Since $\mathfrak{A}$ is $\omega$-categorical, there are finitely many orbits of $n$-tuples in $\mathfrak{A}$, which implies that there are finitely many first-order reducts of $\mathfrak{A}$ up to interdefinability that are bidefinable with $\mathfrak{B}$.

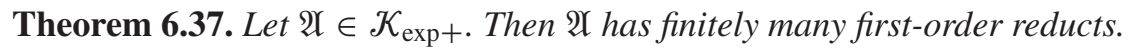

Proof. Follows from Theorem 6.34 and Proposition 6.36. 


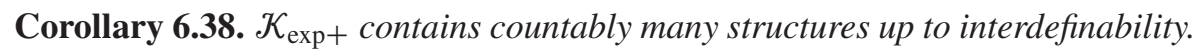
It contains no structure with essentially infinite signature.

Proof. The first statement is implied by Lemma 6.32 in combination with Proposition 6.36. The second statement follows from Theorem 6.37 and Lemma 6.35.

\section{Exponential orbit growth and reducts of unary structures}

In this section, we show that $\mathcal{K}_{\exp }=R(\mathcal{U})$.

Lemma 7.1. $R(\mathcal{U}) \subseteq \mathcal{K}_{\exp }$.

Proof. Let $\mathfrak{A} \in \mathcal{U}$, and let $O_{1}, \ldots, O_{k}$ be the orbits of Aut( $\left.\mathscr{U}\right)$. Then we have $\operatorname{Aut}(\mathfrak{H})=\prod_{i=1}^{k} \operatorname{Sym}\left(O_{i}\right)$ by Lemma 3.1. Hence, the number of injective $n$-orbits of $\operatorname{Aut}(\mathfrak{U})$ is at most $k^{n}$. This implies that $\mathfrak{U} \in \mathcal{K}_{\exp }$, and hence $U \subseteq \mathcal{K}_{\text {exp }}$. The statement follows from the fact that the class $\mathcal{K}_{\text {exp }}$ is closed under taking first-order reducts.

Lemma 7.2. Let $\mathfrak{Q} \in \mathcal{K}_{\exp }$. Then $\Delta(\mathfrak{U})$ is trivial on each infinite orbit of $\operatorname{Aut}(\mathfrak{U})$.

Proof. Let us apply Lemma 6.7 for $G=\operatorname{Aut}(\mathfrak{U}), R=\Delta(\mathfrak{U})$, some $c$ such that $0<c<\frac{1}{2}$, and $k=2$. We obtain that $\Delta(\mathfrak{U})$ has at most finitely many classes of size at least 2. If $O$ is an infinite orbit of $\operatorname{Aut}(\mathfrak{U})$, then every class of $\Delta(\mathfrak{U})$ contained in $O$ has the same size. Therefore, $\Delta(\mathfrak{V})$ must be trivial on each infinite orbit $O$.

Lemma 7.3. Let $\mathfrak{U} \in \mathcal{K}_{\text {exp. }}$ Then we have that $\mathfrak{U}$ is a first-order reduct of some structure $\mathfrak{B} \in \mathcal{K}_{\exp }$ for which $\Delta(\mathfrak{B})$ is trivial.

Proof. Let $F$ be the union of finite orbits of $\mathscr{U}$. Then we have that $F$ is finite. Let $\mathfrak{B}$ be a structure obtained from $\mathfrak{A}$ by adding a constant for each element of $F$. Then $\operatorname{Aut}(\mathfrak{B})=\left.\operatorname{Aut}(\mathfrak{U})\right|_{F}$, and it is easy to see that $\left.\operatorname{Aut}(\mathfrak{U})\right|_{F} \in \mathscr{E}_{\text {exp }}$. Therefore, $\mathfrak{B} \in \mathcal{K}_{\text {exp }}$. Now let $C$ be a class of $\Delta(\mathfrak{B})$. If $C$ is contained in a finite orbit of $\mathfrak{A}$, then by definition, $|C|=1$, and if $C$ is contained in an infinite orbit of $\mathfrak{U}$, then $|C|=1$ by Lemma 7.2. Therefore, $\Delta(\mathfrak{B})$ is trivial.

Theorem 7.4. $\mathcal{K}_{\exp }=R(\mathcal{U})$.

Proof. The containment " $\supseteq$ " is Lemma 7.1. Now assume that $\mathfrak{U} \in \mathcal{K}_{\text {exp }}$. By Lem-

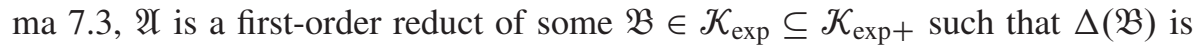
trivial. By Corollary 6.27, we obtain that $\mathfrak{B} \in R(\mathcal{U})$. Hence,

$$
\mathfrak{U} \in(R \circ R)(\mathcal{U})=R(\mathcal{U}) .
$$




\section{Additional descriptions of the classes $\mathcal{K}_{\text {exp }}$ and $\mathcal{K}_{\text {exp+ }}$}

In this section, we present additional descriptions of the class $\mathcal{K}_{\text {exp }}$ that follow from our main results.

\subsection{Generating $\mathcal{K}_{\exp +}$ from $s$}

In this subsection, we show that $\mathcal{K}_{\text {exp }+}$ is the smallest class that contains $\delta$ and is closed under taking first-order reducts, finite covering structures, and adding constants.

Lemma 8.1. The following inclusions hold.

(1) $U^{*} \subseteq(R \circ F \circ C)(8)$.

(2) $u_{\mathrm{nf}} \subseteq(R \circ F)(\mathbb{N})$.

Proof. Let $\mathfrak{U} \in \mathcal{U}^{*}$, and let $O_{1}, \ldots, O_{m}$ be the orbits of Aut( $\left.\mathfrak{U}\right)$ so that

$$
O_{1}=\left\{y_{1}\right\}, \ldots, O_{l}=\left\{y_{l}\right\}
$$

are the finite orbits. Let $F=\left\{y_{1}, \ldots, y_{l}\right\}$. Pick a bijection $b_{i}$ between $O_{i}$ and $\mathbb{N}$ for each $i \in\{l+1, \ldots, m\}$. Let $b=\bigcup_{i=l+1}^{m} b_{i}$, and let

$$
E:=\left\{(x, y) \mid x, y \in \bigcup_{i=l+1}^{m} O_{i}, b(x)=b(y)\right\} .
$$

Let $\mathfrak{C}$ be the structure $\mathfrak{A}$ expanded by the relation $E$. Then

$$
\Delta(\mathfrak{C})=E \cup\{(x, x) \mid x \in F\} \quad \text { and } \operatorname{Aut}(\mathfrak{C} / \Delta(\mathfrak{C}))=\operatorname{Sym}(C / \Delta(\mathfrak{C}))_{F} .
$$

Therefore, $\mathfrak{C} / \Delta(\mathfrak{C}) \in C(\mathscr{\&})$, which shows (1). If $\mathfrak{U}$ has no finite orbits, then $l=0$ and $\mathfrak{S} / \Delta(\mathfrak{E})$ is bi-definable with $\mathbb{N}$, which shows (2).

Lemma 8.2. The classes $\mathcal{K}_{\exp }$ and $\mathcal{K}_{\exp }+$ are closed under $C$.

Proof. We need to show that if a permutation group $G$ on $X$ is in $\mathcal{E}_{\exp }$ or in $\boldsymbol{E}_{\exp +\text {, }}$ then so is $G_{F}$ for any finite $F \subset X$. In the case of $\mathscr{G}_{\exp }$, this is clear. For the class $\mathcal{E}_{\text {exp+, }}$, this is stated in Lemma 6.3 .

Lemma 8.3. For any class $C$ of structures,

$$
\begin{aligned}
(F \circ R)(\mathcal{C}) & \subseteq(R \circ F)(\mathcal{C}), \\
\left(F \circ R^{<\infty}\right)(\mathcal{C}) & \subseteq\left(R^{<\infty} \circ F\right)(\mathcal{C}) .
\end{aligned}
$$


Proof. Let $\mathfrak{C}$ be a structure, let $\mathfrak{B}$ be a first-order reduct of $\mathfrak{C}$, and let $\pi: \mathfrak{A} \rightarrow \mathfrak{B}$ be a finite cover. Let $G:=\operatorname{Aut}(\mathfrak{U}) \cap \mu_{\pi}^{-1}(\operatorname{Aut}(\mathfrak{C}))$. Then $G$ is closed. So $G$ is the automorphism group of some first-order expansion $\mathfrak{D}$ of $\mathfrak{A}$, and $\pi: \mathfrak{D} \rightarrow \mathfrak{C}$ is a finite cover. Hence, $\mathfrak{U} \in(R \circ F)(\mathfrak{C})$. Moreover, if $\mathfrak{E}$ is $\omega$-categorical and $[\operatorname{Aut}(\mathfrak{B}): \operatorname{Aut}(\mathfrak{C})]$ is finite, that is, $\operatorname{Aut}(\mathfrak{B})=g_{1} \operatorname{Aut}(\mathfrak{C}) \cup \cdots \cup g_{n} \operatorname{Aut}(\mathfrak{C})$ for some $g_{1}, \ldots, g_{n} \in \operatorname{Aut}(\mathfrak{B})$, then $\operatorname{Aut}(\mathfrak{Q})=h_{1} \operatorname{Aut}(\mathfrak{C}) \cup \cdots \cup h_{n} \operatorname{Aut}(\mathfrak{C})$ for some $h_{i}$ so that $\mu_{\pi}\left(h_{i}\right)=g_{i}$. In particular, $[\operatorname{Aut}(\mathfrak{H}): \operatorname{Aut}(\mathfrak{D})]$ is finite.

If $\mathfrak{U}$ has no finite orbits, then every first-order reduct of $\mathfrak{U}$ does not have finite orbits, too, so $R\left(\mathcal{C}_{\mathrm{nf}}\right) \subseteq(R(\mathcal{C}))_{\mathrm{nf}}$ for any class $\mathcal{C}$. For $\mathcal{C}=U$, we even get that

$$
R\left(U_{\mathrm{nf}}\right)=R(\mathcal{U})_{\mathrm{nf}}
$$

(see Corollary 3.7). Since a finite covering structure $\mathfrak{A}$ of $\mathfrak{B}$ has finite orbits if and only if $\mathfrak{B}$ has finite orbits, we have for any class $\mathcal{C}$ of structures that

$$
F\left(\mathcal{C}_{\mathrm{nf}}\right)=(F(\mathcal{C}))_{\mathrm{nf}} .
$$

Theorem 8.4. The following equalities hold.

(1) $\mathcal{K}_{\exp +}=(R \circ F \circ C)(8)$.

(2) $\left(\mathcal{K}_{\text {exp }+}\right)_{\mathrm{nf}}=(R \circ F)(\mathbb{N})$.

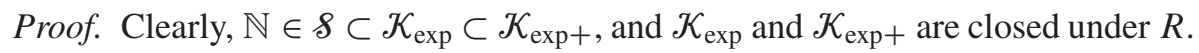
The closure of $\mathcal{K}_{\exp +}$ under $F$ follows from $\mathcal{K}_{\exp +}=(F \circ R)\left(U^{*}\right)$ (see Theorem 6.29), and the closure under $C$ is stated in Lemma 8.2. Finally, $\left(\mathcal{K}_{\exp +}\right)_{\mathrm{nf}}$ is closed under $R$ since $R\left(\left(\mathcal{K}_{\text {exp }+}\right)_{\mathrm{nf}}\right) \subseteq\left(R\left(\mathcal{K}_{\exp +}\right)\right)_{\mathrm{nf}}=\left(\mathcal{K}_{\exp +}\right)_{\mathrm{nf}}$. This shows the inclusions $\supseteq$ in (1) and in (2). For the converse containments, observe that

$$
\begin{array}{rlrl}
\mathcal{K}_{\exp +} & =(F \circ R)\left(\mathcal{U}^{*}\right) & & \text { (by Theorem 6.29) } \\
& \subseteq(F \circ R \circ R \circ F \circ C)(8) & (\text { by Lemma 8.1 (1) }) \\
& =(F \circ R \circ F \circ C)(8) & \\
& \subseteq(R \circ F \circ F \circ C)(8) & & (\text { by }(8.1) \text { in Lemma } \\
& =(R \circ F \circ C)(8), &
\end{array}
$$

which shows (1). Moreover, to show (2),

$$
\begin{aligned}
\left(\mathcal{K}_{\exp +}\right)_{\mathrm{nf}} & =\left((F \circ R)\left(U^{*}\right)\right)_{\mathrm{nf}} & & (\text { by Theorem 6.29) } \\
& =F\left((R(U))_{\mathrm{nf}}\right) & & (\text { by }(8.3)) \\
& =(F \circ R)\left(U_{\mathrm{nf}}\right) & & (\text { by }(8.2)) \\
& \subseteq(F \circ R \circ R \circ F)(\mathbb{N}) & & (\text { by Lemma 8.1 (2)) } \\
& \subseteq(R \circ F)(\mathbb{N}) & & (\text { as above) } .
\end{aligned}
$$




\subsection{Model-complete cores}

The model-complete core of an $\omega$-categorical structure has already been defined in the introduction. In this section, we show that $\mathcal{K}_{\exp }+$ is the smallest class of structures that contains $\mathbb{N}$ and is closed under taking first-order reducts, finite covers, and model-complete cores.

Lemma 8.5. Let $\mathfrak{A}$ be an $\omega$-categorical structure and $\mathfrak{B}$ its model-complete core. Then $o_{n}(\mathfrak{B}) \leq o_{n}(\mathfrak{U})$ and $o_{n}^{i}(\mathfrak{B}) \leq o_{n}^{i}(\mathfrak{U})$ for all $n \in \mathbb{N}$.

Proof. For $o_{n}$, this is [9, Proposition 3.6.24]. The statement for $o_{n}^{i}$ can be shown analogously.

Corollary 8.6. The classes $\mathcal{K}_{\exp }$ and $\mathcal{K}_{\exp }$ are closed under $M$.

Remark 8.7. Analogous statements hold for the model companion instead of the model-complete core.

Definition 8.8. Let $\mathfrak{U}$ be a structure with signature $\tau$, and let $F \subseteq A$. Then let $\mathfrak{A}(F)$ denote the following $\tau$-structure.

- The domain of $\mathfrak{U}(F)$ is $A(F):=(F \times \mathbb{N}) \sqcup((A \backslash F) \times\{0\})$.

- For each $R \in \tau$ of arity $k$, the relation $R^{\mathfrak{A}(F)}$ is defined as

$$
\left\{\left(\left(x_{1}, n_{1}\right), \ldots,\left(x_{k}, n_{k}\right)\right) \mid\left(x_{1}, \ldots, x_{n}\right) \in R\right\} .
$$

Remark 8.9. The map $f: A(F) \rightarrow A$ defined by $(x, n) \mapsto x$ is a homomorphism from $\mathfrak{U}(F)$ to $\mathfrak{A}$. Conversely, the mapping $g: A \rightarrow A(F)$ defined by $x \mapsto(x, 0)$ is a homomorphism from $\mathfrak{A}$ to $\mathfrak{A}(F)$ (in fact, it is an embedding). Therefore, $\mathfrak{A}$ and $\mathfrak{U}(F)$ are homomorphically equivalent.

Remark 8.10. It follows directly from the definition that if $\mathfrak{A}$ is a first-order reduct of $\mathfrak{B}$, and $F \subseteq A$, then $\mathfrak{U}(F)$ is a first-order reduct of $\mathfrak{B}(F)$ (since we can use the same definitions).

Lemma 8.11. Let $\mathfrak{A} \in \mathcal{U}^{*}$, and let $F$ be the union of the finite orbits of $\mathfrak{X}$. Then $\mathfrak{A}(F) \in \mathcal{U}_{\mathrm{nf}}$.

Proof. Let $O_{1}, \ldots, O_{k}$ be the orbits of $\operatorname{Aut}(\mathfrak{U})$. Then $\operatorname{Aut}(\mathfrak{U})=\prod_{i=1}^{k} \operatorname{Sym}\left(O_{i}\right)$ by Lemma 3.1. Let $O_{1}=\left\{y_{1}\right\}, \ldots, O_{l}=\left\{y_{l}\right\}$ be the finite orbits of Aut(2). Then $\operatorname{Aut}(\mathfrak{U}(F))=\prod_{i=1}^{l} \operatorname{Sym}\left(\left\{y_{i}\right\} \times \mathbb{N}\right) \times \prod_{i=l+1}^{k} \operatorname{Sym}\left(O_{i} \times\{0\}\right)$ has no finite orbits, and therefore $\mathfrak{A}(F) \in \mathcal{U}_{\mathrm{nf}}$. 
Lemma 8.12. Let $\mathfrak{A} \in R(\mathcal{U})$, and let $F$ be the union of the finite orbits of $\mathfrak{A}$. Then $\mathfrak{U}(F) \in R(\mathcal{U})_{\mathrm{nf}}$.

Proof. Let $C_{1}, \ldots, C_{n}$ be the classes of $\nabla(\mathfrak{U})$. Then $\prod_{i=1}^{n} \operatorname{Sym}\left(C_{i}\right) \subseteq \operatorname{Aut}(\mathfrak{U})$ by Lemma 3.3, and hence $\mathfrak{A}$ is a first-order reduct of $\mathfrak{B} \in U^{*}$. Lemma 8.11 implies that $\mathfrak{B}(F) \in \mathcal{U}_{\text {nf }}$. Remark 8.10 implies that $\mathfrak{U}(F)$ is a first-order reduct of $\mathfrak{B}(F)$. Hence, $\mathfrak{A}(F) \in R\left(U_{\mathrm{nf}}\right)=R(\mathcal{U})_{\mathrm{nf}}$.

Corollary 8.13. Every structure $\mathfrak{U} \in R(\mathcal{U})$ is interdefinable with a model-complete core of a structure in $R(\mathcal{U})_{\mathrm{nf}}$, i.e., $R(U) \subseteq M\left(R(U)_{\mathrm{nf}}\right)$.

Proof. Let $\mathfrak{X}^{*}$ be the expansion of $\mathfrak{A}$ by all first-order definable relations. Then $\mathfrak{A} *$ is a model-complete core and interdefinable with $\mathfrak{A}$. Let $F$ be the union of the finite orbits of $\operatorname{Aut}\left(\mathfrak{P}^{*}\right)=\operatorname{Aut}(\mathfrak{U})$. By Lemma 8.12, we know that $\mathfrak{X}^{*}(F) \in R(\mathcal{U})_{\mathrm{nf}}$. Since $\mathfrak{U}^{*}$ and $\mathfrak{U}^{*}(F)$ are homomorphically equivalent, it follows that $\mathfrak{U}^{*}$ is the model-complete core of $\mathfrak{U}^{*}(F)$. Hence, $\mathfrak{U} \in M\left(R(\mathcal{U})_{\mathrm{nf}}\right)$.

Corollary 8.14. Let $\mathfrak{B} \in R\left(\mathcal{U}^{*}\right)$, and let $\pi: \mathfrak{U} \rightarrow \mathfrak{B}$ be a finite cover. Then $\mathfrak{X}$ is interdefinable with a model-complete core of a structure in $F\left(R\left(U^{*}\right)\right)_{\mathrm{nf}}$, i.e.,

$$
F\left(R\left(U^{*}\right)\right) \subseteq M\left(F\left(R\left(U^{*}\right)\right)_{\mathrm{nf}}\right) .
$$

Proof. As in the previous proof, let $\mathfrak{U}^{*}$ be the expansion of $\mathfrak{U}$ by all relations that are first-order definable in $\mathfrak{A}$, and let $F$ be the union of the finite orbits of $\operatorname{Aut}(\mathfrak{U})=\operatorname{Aut}\left(\mathfrak{Q}^{*}\right)$. Then $\pi(F)$ is the union of finite orbits of Aut( $\left.\mathfrak{B}\right)$. By Corollary 8.12, we know that $\mathfrak{B}(\pi(F)) \in R(\mathcal{U})$. Let $\pi^{\prime}: A(F) \rightarrow B(\pi(F))$ be defined as

$$
\pi^{\prime}(x, n):= \begin{cases}(\pi(x), n) & \text { if } x \in F, \\ (\pi(x), 0) & \text { otherwise. }\end{cases}
$$

Then it is easy to see that $\pi^{\prime}: \mathfrak{Q}(F) \rightarrow \mathfrak{B}(\pi(F))$ is a finite covering map. Hence, $\mathfrak{U}(F) \in F\left(R\left(\mathcal{U}^{*}\right)\right)$. By Lemma 8.11, the structure $\mathfrak{A}(F)$ has no finite orbits, and as before, we can conclude that $\mathfrak{A}$ is the model-complete core of $\mathfrak{A}(F)$.

Lemma 8.15. The following identities hold.

(1) $\mathcal{K}_{\exp }=M\left(\left(\mathcal{K}_{\exp }\right)_{\mathrm{nf}}\right)$.

(2) $\mathcal{K}_{\exp +}=M\left(\left(\mathcal{K}_{\exp +}\right)_{\mathrm{nf}}\right)$.

(3) $\mathcal{K}_{\exp +}=(M \circ R \circ F)(\mathbb{N})$. 
Proof. The containments " $\supseteq$ " in item (1) and item (2) follow from Corollary 8.6. By Theorems 7.4 and 6.29, we know that $\mathcal{K}_{\text {exp }}=R(\mathcal{U})$ and $\mathcal{K}_{\exp +}=F(R(\mathcal{U})$ ). Then the containments " $\subseteq$ " in item (1) and item (2) follow from Corollaries 8.13 and 8.14. To show item (3), observe that

$$
\begin{aligned}
\mathcal{K}_{\text {exp }+}=M\left(\left(\mathcal{K}_{\exp +}\right)_{\mathrm{nf}}\right) & \text { (by item (2) of the lemma) } \\
& =M(R(F(\mathbb{N}))) \quad \text { (by item (2) of Theorem 8.4) }
\end{aligned}
$$

\subsection{Summary}

The following theorem summarises some of the equivalent characterisations of the classes $\mathcal{K}_{\exp }, \mathcal{K}_{\exp +},\left(\mathcal{K}_{\exp }\right)_{\mathrm{nf}},\left(\mathcal{K}_{\exp +}\right)_{\mathrm{nf}}$.

\section{Theorem 8.16.}

$$
\begin{aligned}
\mathcal{K}_{\exp } & =R(\mathcal{U})=R^{<\infty}\left(U^{*}\right), \\
\left(\mathcal{K}_{\exp }\right)_{\mathrm{nf}} & =R\left(\mathcal{U}_{\mathrm{nf}}\right)=R^{<\infty}\left(U_{\mathrm{nf}}\right), \\
\mathcal{K}_{\exp +} & =(F \circ R)(\mathcal{U})=(R \circ F)(U), \\
& =\left(F \circ R^{<\infty}\right)\left(\mathcal{U}^{*}\right)=\left(R^{<\infty} \circ F\right)\left(U^{*}\right) \\
& =(R \circ F \circ C)(s)=(M \circ R \circ F)(\mathbb{N}), \\
\left(\mathcal{K}_{\exp +}\right)_{\mathrm{nf}} & =(R \circ F)(\mathbb{N})=(F \circ R)\left(U_{\mathrm{nf}}\right) \\
& =\left(F \circ R^{<\infty}\right)\left(U_{\mathrm{nf}}\right)=\left(R^{<\infty} \circ F\right)\left(U_{\mathrm{nf}}\right) .
\end{aligned}
$$

Proof. (8.4): Corollary 3.4 states that $R(U)=R^{<\infty}\left(U^{*}\right)$ and Theorem 7.4 that $\mathcal{K}_{\exp }=R(U)$.

(8.5): We have $R^{<\infty}\left(\mathcal{U}_{\mathrm{nf}}\right) \subseteq R\left(\mathcal{U}_{\mathrm{nf}}\right)=R(\mathcal{U})_{\mathrm{nf}}=\left(\mathcal{K}_{\exp }\right)_{\mathrm{nf}}$ by $(8.2)$ and $(8.4)$, and $R(\mathcal{U})_{\mathrm{nf}} \subseteq R^{<\infty}\left(\mathcal{U}_{\mathrm{nf}}\right)$ can be shown as in the proof of Corollary 3.4.

(8.6): By Theorem 6.29, we know that

$$
\mathcal{K}_{\exp +}=(F \circ R)(U)=\left(R^{<\infty} \circ F\right)\left(U^{*}\right) .
$$

This also implies that the class $\mathcal{K}_{\exp +}$ is closed under $F$, and it is obviously closed under $R$, so

$$
\mathcal{K}_{\exp +}=\left(R^{<\infty} \circ F\right)\left(\mathcal{U}^{*}\right) \subseteq(R \circ F)(\mathcal{U}) \subseteq \mathcal{K}_{\exp +} .
$$

The equality $(F \circ R)(U)=\left(F \circ R^{<\infty}\right)\left(U^{*}\right)$ follows from $R(U)=R^{<\infty}\left(U^{*}\right)$ (Corollary 3.4). The equality $\mathcal{K}_{\exp +}=(R \circ F \circ C)(8)$ is item (1) of Theorem 8.4, and the equality $\mathcal{K}_{\exp +}=(M \circ R \circ F)(\mathbb{N})$ is item (3) of Lemma 8.15.

(8.7): The proof of Theorem 8.4 (2) shows the following equalities:

$$
\left(\mathcal{K}_{\exp +}\right)_{\mathrm{nf}}=(F \circ R)\left(\mathcal{U}_{\mathrm{nf}}\right)=(R \circ F)(\mathbb{N}) .
$$


Finally,

$$
\begin{aligned}
\left(\mathcal{K}_{\exp +}\right)_{\mathrm{nf}}=(F \circ R)\left(\mathcal{U}_{\mathrm{nf}}\right) & =\left(F \circ R^{<\infty}\right)\left(\mathcal{U}_{\mathrm{nf}}\right) \quad \text { (as in Corollary 3.4) } \\
& \subseteq\left(R^{<\infty} \circ F\right)\left(\mathcal{U}_{\mathrm{nf}}\right) \quad \text { (by (8.1)) } \\
& \subseteq\left(\mathcal{K}_{\exp +}\right)_{\mathrm{nf}},
\end{aligned}
$$

and thus $\left(\mathcal{K}_{\exp +}\right)_{\mathrm{nf}}=\left(F \circ R^{<\infty}\right)\left(\mathcal{U}_{\mathrm{nf}}\right)=\left(R^{<\infty} \circ F\right)\left(\mathcal{U}_{\mathrm{nf}}\right)$.

\section{Consequences for constraint satisfaction}

In the introduction, we have already mentioned that, for finite structures $\mathfrak{A}$, there is a complexity dichotomy for $\operatorname{CSP}(\mathfrak{A})$ : these problems are in $\mathrm{P}$ or NP-complete. Such a complexity dichotomy has also been conjectured for the much larger class of first-order reducts of finitely bounded homogeneous structures. A structure $\mathfrak{B}$ with finite relational signature $\tau$ is called finitely bounded if there exists a finite set of finite $\tau$-structures $\mathscr{F}$ such that a finite $\tau$-structure $\mathfrak{U}$ embeds into $\mathfrak{B}$ if and only if no structure from $\mathscr{F}$ embeds into $\mathfrak{A}$. For first-order reducts of finitely bounded homogeneous structures, there is also a more specific infinite-domain tractability conjecture [21]: assuming that $\mathfrak{U}$ is a model-complete core, the conjecture says that $\operatorname{CSP}(\mathfrak{A})$ is in $\mathrm{P}$ if and only if $\mathfrak{U}$ has a pseudo-Siggers polymorphism (for a definition of pseudo-Siggers polymorphisms and a proof that the conjecture can be phrased like this, see [6]).

Let $\mathfrak{U}$ be a structure from $\mathcal{K}_{\exp +}$ with finite relational signature. The next lemma shows that the question whether CSP( $\mathfrak{A})$ is in P or NP-complete falls into the scope of this conjecture.

Lemma 9.1. Every structure in $\mathcal{K}_{\exp }+$ is a first-order reduct of a finitely bounded homogeneous structure.

Proof. Let $\mathfrak{U} \in \mathcal{K}_{\exp +}$. By Theorem 6.29, we have $\mathcal{K}_{\exp +}=R\left(F\left(\mathcal{U}^{*}\right)\right)$, so $\mathfrak{A}$ is a first-order reduct of a structure $\mathfrak{Q}^{\prime} \in F\left(\mathcal{U}^{*}\right)$. By Proposition 4.9, every finite cover of a structure in $U^{*}$ is strongly split, so we can assume that $\mathfrak{U}^{\prime}$ is a strongly trivial covering structure of a structure $\mathfrak{B} \in U^{*}$. Let $\mathfrak{C}$ be the structure from the proof of Lemma 5.4, and let $\tau:=\left(\left\{U_{i, s} \mid i \leq k, s \in F_{i}\right\} \cup\left\{\sim_{\pi}\right\}\right)$ be the signature of $\mathfrak{C}$. Then it is easy to specify a finite set of forbidden finite $\tau$-structures such that, in any finite $\tau$-structure that avoids these structures,

- the relation $\sim_{\pi}$ is an equivalence relation,

- the sets denoted by the unary relations $U_{i, s}$ are pairwise disjoint and cover all of $C$, 
- for all $i, s$, if $x \sim_{\pi} y$ and $x, y \in U_{i, s}$, then $x=y$,

- for all $i, s$, the cardinality of $U_{i, j}$ is at most the cardinality of $U_{i, j}$ in $\mathfrak{E}$.

These are precisely the finite structures that embed into $\mathfrak{C}$.

Let $\mathfrak{A}$ be a structure from $R(F(U)$ ). In this section, we discuss the consequences of our results for classifying the computational complexity of $\operatorname{CSP}(\mathfrak{U})$. First, since $R(F(U))=\mathcal{K}_{\text {exp }}$ is closed under $M$ as discussed above, we can assume that $\mathfrak{U}$ is a model-complete core. The following lemma shows that we can even assume that $\mathfrak{A} \in F(\mathcal{U})$.

Lemma 9.2. Let $\mathfrak{U} \in R(F(\mathcal{U}))$. Then there exists a model-complete core $\mathfrak{C}$ in $F\left(U^{*}\right)$ such that

- $\operatorname{CSP}(\mathfrak{U})$ and $\operatorname{CSP}(\mathfrak{C})$ are polynomial-time equivalent,

- the $\nabla(\mathfrak{C})$-classes are the orbits of $\operatorname{Aut}(\mathfrak{C})$ and they are primitively positively definable in $\mathfrak{E}$.

Proof. Let $\mathfrak{C}^{\prime}$ be the model-complete core of $\mathfrak{A}$. Then $\mathfrak{C}^{\prime}$ is in

$$
M(R(F(U)))=\mathcal{K}_{\exp +}=F(R(\mathcal{U})) .
$$

So suppose that $\pi: \mathbb{C}^{\prime} \rightarrow \mathfrak{B}^{\prime}$ is a finite covering for $\mathfrak{B}^{\prime} \in R(\mathcal{U})$. Add a constant $c$ from each $\nabla\left(\mathfrak{C}^{\prime}\right)$-equivalence class to $\mathfrak{C}^{\prime}$, and let $\mathfrak{C}$ be the resulting structure. Then $\mathfrak{C}$ is still $\omega$-categorical and a model-complete core. Moreover, $\mathfrak{C}$ and $\mathfrak{A}$ are polynomial-time equivalent [8].

Add a constant $\pi(c)$ to $\mathfrak{B}^{\prime}$ for each of the new constants $c$, and let $\mathfrak{B}$ be the structure obtained in this way. The proof of Corollary 3.5 shows that $\mathfrak{B} \in \mathcal{U}^{*}$. Then $\pi: \mathfrak{C} \rightarrow \mathfrak{B}$ is a finite cover. Therefore, $\mathfrak{C} \in F\left(\mathcal{U}^{*}\right)$. Moreover, the $\nabla(\mathfrak{C})$ classes are the orbits of $\mathfrak{C}$, and orbits in model-complete cores are primitive positive definable [8].

It can be shown using the universal-algebraic approach to constraint satisfaction that if $\mathfrak{C} \in F(R(U))$, then CSP( $(\mathfrak{C})$ is either in P or NP-complete. This lies beyond the scope of this article, but will appear elsewhere.

\section{Conclusion and open problems}

Our results imply that all structures in $\mathcal{K}_{\text {exp }}$ are $\omega$-stable (Remark 6.30), that they are first-order reducts of finitely bounded homogeneous structures (Proposition 9.1), and that they satisfy Thomas' conjecture (Corollary 6.37). Do $\omega$-stable homogeneous structures with finite relational signature in general satisfy Thomas' 
conjecture, i.e., do they have finitely many reducts up to interdefinability? Note that if we drop the assumption about having a relational signature, then the answer is "no" even if we insist on $\mathfrak{A}$ being still $\omega$-categorical and $\omega$-stable (this follows from the example given in [23], which is the expansion of the countably infinite dimensional vector space over the two-element field with one non-zero constant).

Answering the question of the previous paragraph might be very ambitious, so we propose to first study a more concrete and fundamental class of structures. Let $\mathcal{K}=$ be the class of all structures with a first-order interpretation over $(\mathbb{N} ;=)$ (which we have already discussed in Section 1.2). Do the structures in $\mathcal{K}=$ satisfy Thomas' conjecture? Is the model companion of a structure in $\mathcal{K}=$ also in $\mathcal{K}=$ ? We ask the same question for the model-complete cores of structures in $\mathcal{K}=$.

By our results, structures from $\mathcal{K}_{\exp }+$ can be represented on a computer as follows. First, every trivial covering $\mathfrak{A}$ of a structure $\mathfrak{B} \in \mathcal{U}$ is interdefinable with a homogeneous structure in a finite relational signature $\mathfrak{C}$ (Proposition 5.4) and is finitely bounded (Lemma 9.1). So we can represent $\mathfrak{C}$ up to isomorphism by specifying these bounds. Second, finite-signature first-order reducts of $\mathbb{5}$ can be represented by listing formulas for the relations of the reduct (we can assume that these formulas are quantifier-free since $\mathfrak{C}$ is homogeneous in a finite relational signature and hence has quantifier elimination), and storing these together with the representation for $\mathfrak{C}$. We now ask which of the following problems are algorithmically decidable.

(1) Given two structures in $(R \circ F)(\mathcal{U})$, decide whether they are isomorphic.

(2) Given two structures in $(R \circ F)(\mathcal{U})$, decide whether they are interdefinable.

(3) Given two structures in $(R \circ F)(U)$, decide whether they are bi-interpretable.

Szymon Toruńczyk (personal communication) observed that the first of these questions (about deciding isomorphism of two given structures) is in the larger setting of reducts of finitely bounded homogeneous structures equivalent to an open problem about decidability of first-order definability from [22] (the final open problem mentioned there).

\section{Bibliography}

[1] L. Agarwal, Reducts of the generic digraph, Ann. Pure Appl. Logic 167 (2016), no. 3 , 370-391.

[2] L. Agarwal and M. Kompatscher, $2^{\aleph_{0}}$ pairwise nonisomorphic maximal-closed subgroups of $\operatorname{Sym}(\mathbb{N})$ via the classification of the reducts of the Henson digraphs, J. Symb. Log. 83 (2018), no. 2, 395-415. 
[3] G. Ahlbrandt and M. Ziegler, Quasi-finitely axiomatizable totally categorical theories, Ann. Pure Appl. Logic 30 (1986), 63-82.

[4] G. Ahlbrandt and M. Ziegler, What's so special about $(\mathbf{Z} / 4 \mathbf{Z})^{\omega}$ ?, Arch. Math. Logic 31 (1991), no. 2, 115-132.

[5] R. Akhtar and A. H. Lachlan, On countable homogeneous 3-hypergraphs, Arch. Math. Logic 34 (1995), no. 5, 331-344.

[6] L. Barto and M. Pinsker, The algebraic dichotomy conjecture for infinite domain constraint satisfaction problems, in: Proceedings of the 31st Annual ACM-IEEE Symposium on Logic in Computer Science (LICS 2016), ACM, New York (2016), 615-622.

[7] I. Ben Yaacov and T. Tsankov, Weakly almost periodic functions, model-theoretic stability, and minimality of topological groups, Trans. Amer. Math. Soc. 368 (2016), no. $11,8267-8294$.

[8] M. Bodirsky, Cores of countably categorical structures, Log. Methods Comput. Sci. 3 (2007), no. 1, 1-16.

[9] M. Bodirsky, Complexity classification in infinite-domain constraint satisfaction, Mémoire d'habilitation à diriger des recherches, preprint (2012), https : / /arxiv . org/abs/1201.0856.

[10] M. Bodirsky, Ramsey classes: Examples and constructions, in: Surveys in Combinatorics 2015, London Math. Soc. Lecture Note Ser. 424, Cambridge University, Cambridge (2015), 1-48.

[11] M. Bodirsky, D. Bradley-Williams, M. Pinsker and A. Pongrácz, The universal homogeneous binary tree, J. Logic Comput. 28 (2018), no. 1, 133-163.

[12] M. Bodirsky, M. Hils and B. Martin, On the scope of the universal-algebraic approach to constraint satisfaction, Log. Methods Comput. Sci. 8 (2012), DOI 10.2168/LMCS-8(3:13)2012.

[13] M. Bodirsky, P. Jonsson and T. V. Pham, The reducts of the homogeneous binary branching C-relation, J. Symb. Log. 81 (2016), no. 4, 1255-1297.

[14] M. Bodirsky and J. Kára, The complexity of temporal constraint satisfaction problems, J. ACM 57 (2010), no. 2, Article ID 9.

[15] M. Bodirsky and A. Mottet, A dichotomy for first-order reducts of unary structures, Log. Methods Comput. Sci. 14 (2018), no. 2, Paper No. 13.

[16] M. Bodirsky and J. Nešetřil, Constraint satisfaction with countable homogeneous templates, J. Logic Comput. 16 (2006), no. 3, 359-373.

[17] M. Bodirsky and M. Pinsker, Reducts of Ramsey structures, in: Model Theoretic Methods in Finite Combinatorics, Contemp. Math. 558, American Mathematical Society, Providence (2011), 489-519. 
[18] M. Bodirsky and M. Pinsker, Schaefer's theorem for graphs, J. ACM 62 (2015), no. 3, Article ID 19.

[19] M. Bodirsky and M. Pinsker, Canonical functions: A proof via topological dynamics, preprint (2016), http://arxiv.org/abs/1610.09660.

[20] M. Bodirsky, M. Pinsker and A. Pongrácz, The 42 reducts of the random ordered graph, Proc. Lond. Math. Soc. (3) 111 (2015), no. 3, 591-632.

[21] M. Bodirsky, M. Pinsker and A. Pongrácz, Projective clone homomorphisms, J. Symb. Log. (2019), DOI 10.1017/jsl.2019.23.

[22] M. Bodirsky, M. Pinsker and T. Tsankov, Decidability of definability, J. Symbolic Logic 78 (2013), no. 4, 1036-1054.

[23] B. Bodor, P. J. Cameron and C. Szabó, Infinitely many reducts of homogeneous structures, Algebra Universalis 79 (2018), no. 2, Paper No. 43.

[24] M. Bojańczyk, B. Klin and S. Lasota, Automata theory in nominal sets, Log. Methods Comput. Sci. 10 (2014), no. 3, DOI 10.2168/LMCS-10(3:4)2014.

[25] M. Bojańczyk, B. Klin, S. Lasota and S. Toruńczyk, Turing machines with atoms, in: 2013 28th Annual ACM/IEEE Symposium on Logic in Computer Science (LICS 2013), IEEE Computer Society, Los Alamitos (2013), 183-192.

[26] M. Bojańczyk and S. Toruńczyk, On computability and tractability for infinite sets, in: Proceedings of the 33rd Annual ACM/IEEE Symposium on Logic in Computer Science (LICS), ACM, New York (2018), 145-154.

[27] A. A. Bulatov, A dichotomy theorem for nonuniform CSPs, in: 58th Annual IEEE Symposium on Foundations of Computer Science-FOCS 2017, IEEE Computer Society, Los Alamitos (2017), 319-330.

[28] P. J. Cameron, Transitivity of permutation groups on unordered sets, Math. Z. 148 (1976), no. 2, 127-139.

[29] P. J. Cameron, Normal subgroups of infinite multiply transitive permutation groups, Combinatorica 1 (1981), no. 4, 343-347.

[30] P. J. Cameron, Oligomorphic Permutation Groups, London Math. Soc. Lecture Note Ser. 152, Cambridge University Press, Cambridge, 1990.

[31] P. J. Cameron, Some counting problems related to permutation groups, Discrete Math. 225 (2000), 77-92.

[32] P. J. Cameron, Homogeneous permutations, Electron. J. Combin. 9 (2002-3), no. 2, Research Paper 2.

[33] G. Cherlin, L. Harrington and A. H. Lachlan, $\boldsymbol{\aleph}_{0}$-categorical, $\boldsymbol{\aleph}_{0}$-stable structures, Ann. Pure Appl. Logic 28 (1985), no. 2, 103-135.

[34] G. Cherlin and E. Hrushovski, Finite Structures with Few Types, Ann. of Math. Stud. 152, Princeton University Press, Princeton, 2003. 
[35] G. L. Cherlin, The classification of countable homogeneous directed graphs and countable homogeneous n-tournaments, Mem. Amer. Math. Soc. 131 (1998), no. 621.

[36] J. D. Dixon and B. Mortimer, Permutation Groups, Grad. Texts in Math. 163, Springer, New York, 1996.

[37] J. D. Dixon, P. M. Neumann and S. Thomas, Subgroups of small index in infinite symmetric groups, Bull. Lond. Math. Soc. 18 (1986), no. 6, 580-586.

[38] D. M. Evans, Examples of $\boldsymbol{\aleph}_{0}$-categorical structures, in: Automorphisms of FirstOrder Structures, Oxford Sci. Publ., Oxford Univ. Press, New York (1994), 33-72.

[39] D. M. Evans, D. Macpherson and A. A. Ivanov, Finite covers, in: Model Theory of Groups and Automorphism Groups (Blaubeuren 1995), London Math. Soc. Lecture Note Ser. 244, Cambridge University Press, Cambridge (1997), 1-72.

[40] D. M. Evans and E. Pastori, Second cohomology groups and finite covers of infinite symmetric groups, J. Algebra 330 (2011), 221-233.

[41] T. Feder and M. Y. Vardi, The computational structure of monotone monadic SNP and constraint satisfaction: A study through Datalog and group theory, SIAM J. Comput. 28 (1999), no. 1, 57-104.

[42] M. Gabbay and A. M. Pitts, A new approach to abstract syntax with variable binding, Formal Asp. Comput. 13 (2002), no. 3-5, 341-363.

[43] E. D. Gaughan, Topological group structures of infinite symmetric groups, Proc. Natl. Acad. Sci. USA 58 (1967), 907-910.

[44] C. W. Henson, Countable homogeneous relational structures and $\boldsymbol{\aleph}_{0}$-categorical theories, J. Symbolic Logic 37 (1972), 494-500.

[45] W. Hodges, Model Theory, Encyclopedia Math. Appl. 42, Cambridge University Press, Cambridge, 1993.

[46] W. Hodges, I. Hodkinson, D. Lascar and S. Shelah, The small index property for $\omega$-stable $\omega$-categorical structures and for the random graph, J. Lond. Math. Soc. (2) 48 (1993), no. 2, 204-218.

[47] W. Hodges and A. Pillay, Cohomology of structures and some problems of Ahlbrandt and Ziegler, J. Lond. Math. Soc. (2) 50 (1994), no. 1, 1-16.

[48] E. Hrushovski, Totally categorical structures, Trans. Amer. Math. Soc. 313 (1989), no. $1,131-159$.

[49] T. Jenkinson, J. K. Truss and D. Seidel, Countable homogeneous multipartite graphs, European J. Combin. 33 (2012), no. 1, 82-109.

[50] M. Junker and M. Ziegler, The 116 reducts of $(\mathbb{Q},<, a)$, J. Symbolic Logic 73 (2008), no. 3, 861-884. 
[51] B. Klin, S. Lasota, J. Ochremiak and S. Toruńczyk, Homomorphism problems for first-order definable structures, in: 36th IARCS Annual Conference on Foundations of Software Technology and Theoretical Computer Science (FSTTCS 2016), Leibniz International Proceedings in Informatics (LIPIcs) 65, Schloss Dagstuhl. LeibnizZentrum für Informatik, Wadern (2016), 14:1-14:15.

[52] A. H. Lachlan, Countable homogeneous tournaments, Trans. Amer. Math. Soc. 284 (1984), no. 2, 431-461.

[53] D. C. Lockett and J. K. Truss, Homogeneous coloured multipartite graphs, European J. Combin. 42 (2014), 217-242.

[54] H. D. Macpherson, Growth rates in infinite graphs and permutation groups, Proc. Lond. Math. Soc. (3) 51 (1985), no. 2, 285-294.

[55] H. D. Macpherson, Orbits of infinite permutation groups, Proc. Lond. Math. Soc. (3) 51 (1985), no. 2, 246-284.

[56] H. D. Macpherson, Infinite permutation groups of rapid growth, J. Lond. Math. Soc. (2) 35 (1987), no. 2, 276-286.

[57] J. Melleray, Polish groups and Baire category methods, Confluentes Math. 8 (2016), no. 1, 89-164.

[58] S. Moon and Y. Stalder, Highly transitive actions of free products, Algebr. Geom. Topol. 13 (2013), no. 1, 589-607.

[59] P. P. Pach, M. Pinsker, G. Pluhár, A. Pongrácz and C. Szabó, Reducts of the random partial order, Adv. Math. 267 (2014), 94-120.

[60] G. Paolini and S. Shelah, Reconstructing structures with the strong small index property up to bi-definability, Fund. Math. 247 (2019), no. 1, 25-35.

[61] E. Pastori, On finite covers, groupoids and finite internal covers, Rend. Mat. Appl. (7) 31 (2011), no. 1-2, 1-19.

[62] C. Rosendal, Automatic continuity of group homomorphisms, Bull. Symbolic Logic 15 (2009), no. 2, 184-214.

[63] J. H. Schmerl, Countable homogeneous partially ordered sets, Algebra Universalis 9 (1979), no. 3, 317-321.

[64] S. Thomas, Reducts of the random graph, J. Symbolic Logic 56 (1991), no. 1, 176181.

[65] S. Thomas, Reducts of random hypergraphs, Ann. Pure Appl. Logic 80 (1996), no. 2, 165-193.

[66] D. Zhuk, A proof of CSP dichotomy conjecture, in: 58th Annual IEEE Symposium on Foundations of Computer Science-FOCS 2017, IEEE Computer Society, Los Alamitos (2017), 331-342.

[67] M. Ziegler, Finite covers of disintegrated sets, preprint (1992). 
Received December 11, 2018; revised December 11, 2020.

\section{Author information}

Manuel Bodirsky, Institute of Algebra, TU Dresden, Dresden, Germany.

E-mail: manuel.bodirsky@tu-dresden.de

Bertalan Bodor, Institute of Algebra, TU Dresden, Dresden, Germany.

E-mail: bertalan.bodor@tu-dresden.de 